\title{
Investigation of the Active Site, Regulatory Domain, and Expression System of Cystathionine $\beta$-Synthase
}

\author{
By
}

Faraz Quazi, B. Sc.

\author{
A thesis submitted to \\ the Faculty of Graduate Studies and Research \\ in partial fulfillment of \\ the requirements for the degree of
}

Masters of Science

Biology Department

Ottawa-Carleton Institute of Biology

Carleton University

Ottawa, Ontario

May 2007

Copyright

2008 Faraz Quazi 


$\begin{array}{ll}\begin{array}{l}\text { Library and } \\ \text { Archives Canada }\end{array} & \begin{array}{l}\text { Bibliothèque et } \\ \text { Archives Canada }\end{array} \\ \begin{array}{l}\text { Published Heritage } \\ \text { Branch }\end{array} & \begin{array}{l}\text { Direction du } \\ \text { Patrimoine de l'édition }\end{array} \\ \begin{array}{l}\text { 395 Wellington Street } \\ \text { Ottawa ON K1A 0N4 } \\ \text { Canada }\end{array} & \begin{array}{l}\text { 395, rue Wellington } \\ \text { Ottawa ON K1A 0N4 } \\ \text { Canada }\end{array}\end{array}$

Your file Votre référence ISBN: 978-0-494-44136-7

Our file Notre référence

ISBN: 978-0-494-44136-7

NOTICE:

The author has granted a nonexclusive license allowing Library and Archives Canada to reproduce, publish, archive, preserve, conserve, communicate to the public by telecommunication or on the Internet, loan, distribute and sell theses worldwide, for commercial or noncommercial purposes, in microform, paper, electronic and/or any other formats.

The author retains copyright ownership and moral rights in this thesis. Neither the thesis nor substantial extracts from it may be printed or otherwise reproduced without the author's permission.
AVIS:

L'auteur a accordé une licence non exclusive permettant à la Bibliothèque et Archives Canada de reproduire, publier, archiver, sauvegarder, conserver, transmettre au public par télécommunication ou par l'Internet, prêter, distribuer et vendre des thèses partout dans le monde, à des fins commerciales ou autres, sur support microforme, papier, électronique et/ou autres formats.

L'auteur conserve la propriété du droit d'auteur et des droits moraux qui protège cette thèse. $\mathrm{Ni}$ la thèse ni des extraits substantiels de celle-ci ne doivent être imprimés ou autrement reproduits sans son autorisation.
In compliance with the Canadian Privacy Act some supporting forms may have been removed from this thesis.

While these forms may be included in the document page count, their removal does not represent any loss of content from the thesis.
Conformément à la loi canadienne sur la protection de la vie privée, quelques formulaires secondaires ont été enlevés de cette thèse.

Bien que ces formulaires aient inclus dans la pagination, il n'y aura aucun contenu manquant.

\section{Canada}




\section{ABSTRACT.}

Cystathionine $\beta$-Synthase (CBS) catalyzes the pyridoxal 5'-phosphate (PLP)dependent condensation of L-homocysteine and L-serine to form L-cystathionine. A series of expression constructs, in which human CBS (hCBS) is expressed with a 6-His affinity tag, were created to facilitate the efficient and economical purification of this enzyme. It was determined that a fusion protein partner is not required for the expression of soluble hCBS and that the steady-state kinetic parameters were unaltered by the presence of an amino-terminal, 6-His affinity tag. Site-directed mutants of the residue S289, which is within hydrogen-bonding distance of position $\mathrm{N}-1$ of the pyridine ring of PLP, of the model yeast enzyme were constructed to investigate the manner in which CBS controls the chemistry of this versatile cofactor. The reduction in fluorescence energy transfer, from tryptophan residues to the cofactor, suggests a change in the orientation of the cofactor in both the S289A and S289D mutants. The $\sim 800$-fold decrease in the $k_{c a t} K_{m}{ }^{L-S e r}$ of the S289A mutant suggests that the CBS-catalyzed $\alpha, \beta$-elimination of L-Ser occurs via an $\mathrm{E}_{1}$ mechanism. Although both hCBS and yeast CBS (yCBS) comprise a carboxyterminal, regulatory domain, hCBS is reported to be allosterically activated by $S$-adenosyl methionine (SAM), while yCBS is SAM-insensitive. A series of residues in the regulatory domain of both enzymes were targeted for site-directed mutagenesis based on sequence alignment and the structure of similar domains in other proteins. Residues F443 and D538 were postulated to mediate $\pi$-stacking and hydrogen bonding interactions with the adenine ring and the hydroxyl groups of the ribose ring of SAM, respectively. Although the specific activity of the D538A mutant is similar to the wild-type enzyme and that of F443A is increased by 2.2-fold, neither mutant is activated by SAM. 


\section{ACKNOWLEDGEMENTS.}

I can sum up my experience in a few words: A tough task, but a satisfying one. It is hard to resist that the time to settle the accounts has come. So many people have left an impression on some part or another of this work that I cannot imagine calling all of them to mind to thank them properly. So, I will offer thanks to those to whom I think owe the most obvious debts. Thanks to my friends, who made graduate school at Carleton University a delightful part of my life. Ali Farsi, Nikolai Chepelov, Pratik Lodha, Colleen Woodhouse, Jen Skanes, Justin Kicks, Heidi Los, Navya Kalidindi, Brian Turnbull, and Nikita Rayne all had a hand in this project in some way or another, and I am deeply grateful. I also like to extend my appreciation to Dr. Bill Willmore and Dr. Ken Storey. Besides being terrific advisors, the graduate-level courses I took under them were insightful, thrilling, and very enlightening for my future career. My deepest thanks, though, are to my parents. All that I am or ever hope to be, I owe it to them. In some ways, this thesis is a book about learning, and so I would like to thank my supervisor, Dr. Susan M. Aitken. Sue was my perpetual scholarly resource throughout graduate school, a reader of my thesis, and my constant inspiration for more than three years. Sue's mentoring has given me whatever scholarly issues I have, and I am sure that I will continue to learn from her whatever I have missed along the way. 
3. DEDICATION.

To Mamma and Daddy 


\section{TABLE OF CONTENTS.}

1. ABSTRACT.............................................................................................................................. iii

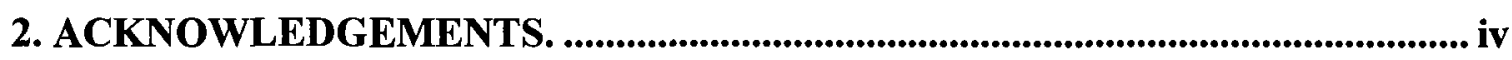

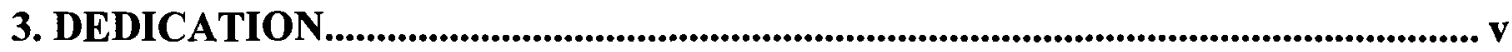

4. TABLE OF CONTENTS. ......................................................................................... vi

5. LIST OF TABLES. ................................................................................................. viii

6. LIST OF FIGURES. ................................................................................................ ix

7. ABBREVIATIONS............................................................................................... xiii

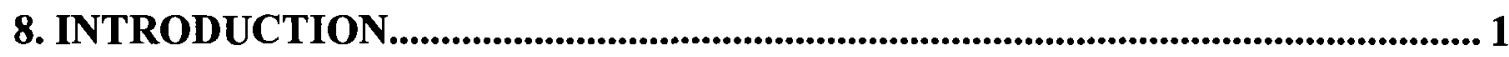

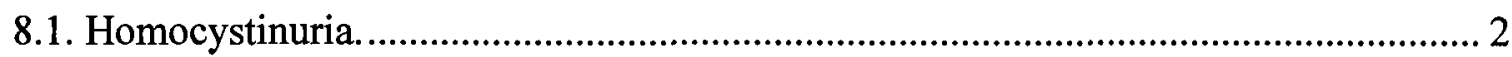

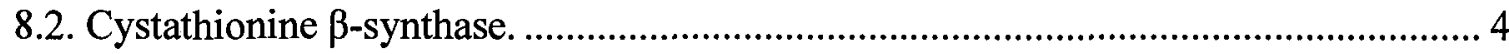

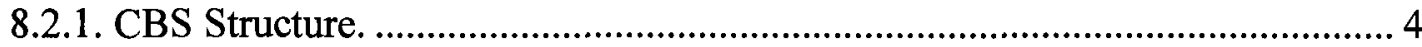

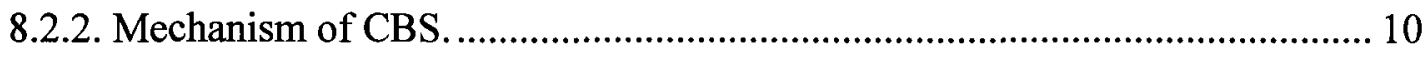

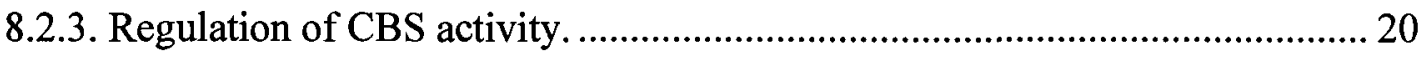

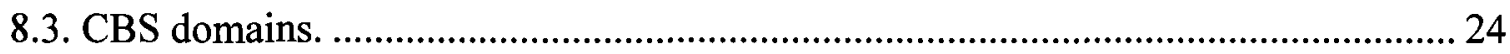

8.3.1. Nucleotide binding in other CBS domain proteins..................................... 28

8.3.1.1. Inosine-5'-monophosphate dehydrogenase. .......................................... 28

8.3.1.2. Chloride-channel protein. ......................................................................... 28

8.3.1.3. AMP-Activated Protein Kinase. ................................................................ 32

8.4. Locating the allosteric-binding site in hCBS......................................................... 33

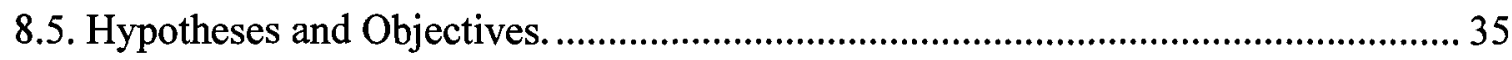

9. Kinetic Characterization of Recombinant Human Cystathionine $\beta$-Synthase

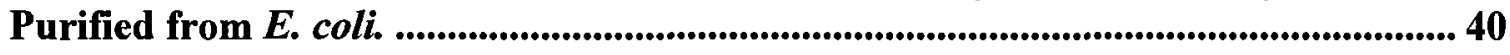

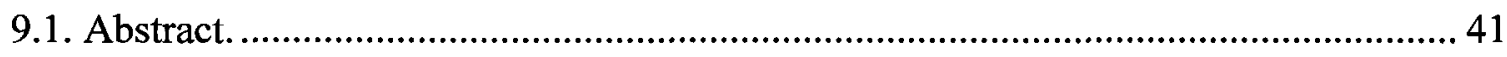

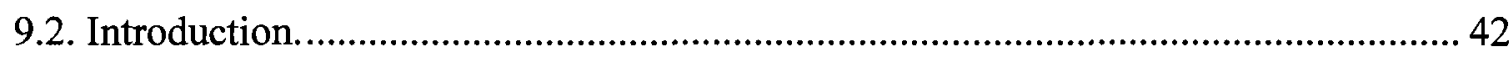

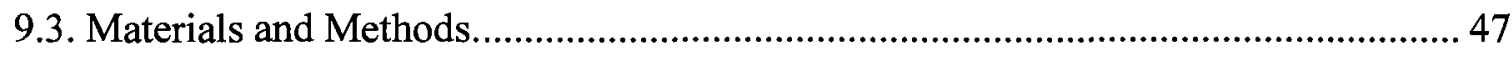

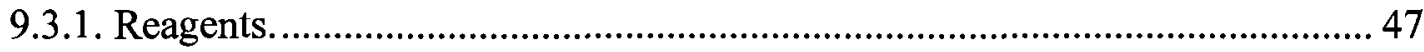

9.3.2. Preparation of the 6-His-GST/hCBS Expression Construct............................ 47

9.3.3. Preparation of the 6-His/hCBS and 6-His-GFP/hCBS Expression Constructs.

9.3.4. Preparation of the hCBS/6-His and 6-His-linker/hCBS Expression Constructs.

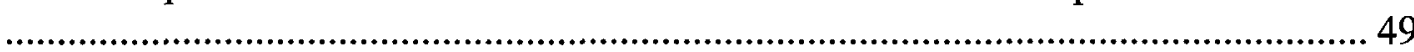

9.3.5. Protein Expression. ...................................................................................... 50

9.3.6. Enzyme Assays. ......................................................................................... 51

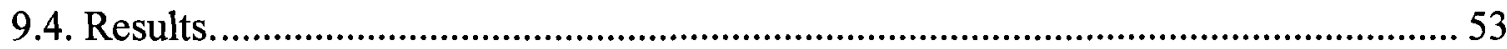

9.4.1. Evaluation of hCBS expression constructs.................................................... 53

9.4.2. Steady-state characterization of hCBS............................................................ 55

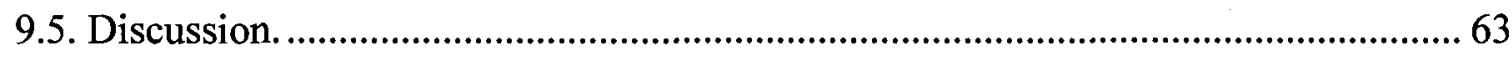




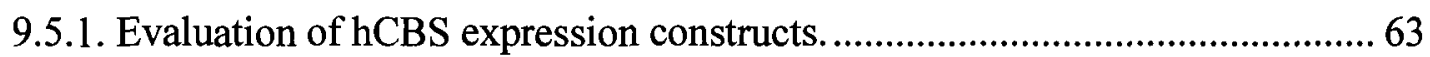

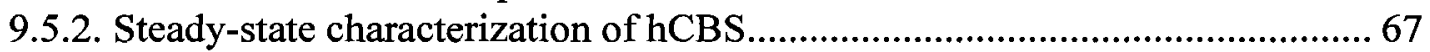

10. Characterization of S289A,D Mutants of Yeast Cystathionine B-Synthase........ 69

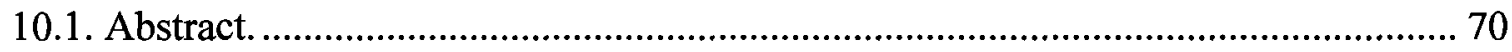

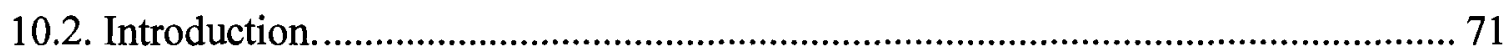

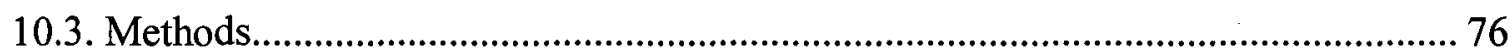

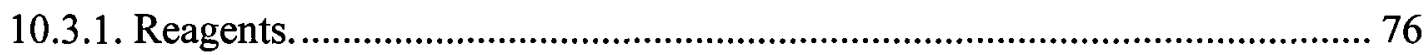

10.3.2. Construction, Expression and Purification of ytCBS-His mutants................ 76

10.3.3. Steady-State Kinetics..................................................................................... 77

10.3.4. Fluorescence Spectroscopy................................................................. 78

10.3.5. Single Turnover Measurements................................................................... 79

10.3.6. Spectrophotometric Titration of the ytCBS Internal Aldimine....................... 80

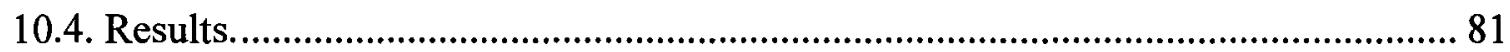

10.4.1. Steady-state kinetic parameters...................................................................... 81

10.4.2. Formation and stability of the aminoacrylate intermediate. ............................ 81

10.4.3. Equilibrium spectroscopic studies. ................................................................. 83

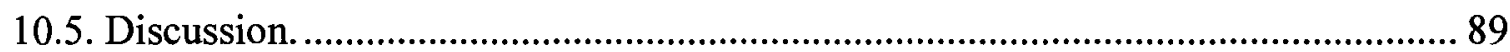

11. Nucleotide recognition by the C-terminal regulatory domain of human Cystathionine $\beta$-Synthase..................................................................................................... 92

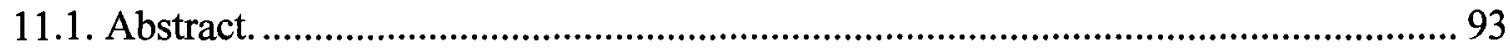

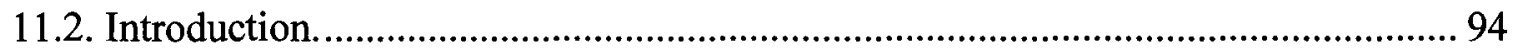

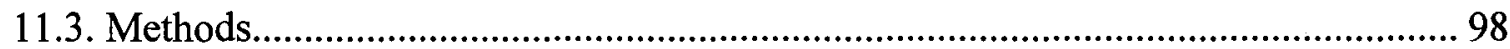

11.3.1. Construction, Expression, and Purification of yeast and human CBS mutants.

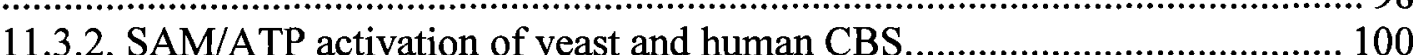

11.3.3. Urea denaturation - yeast CBS stability .................................................. 100

11.3.4. Steady-state kinetics of yeast and human CBS domain mutants................. 101

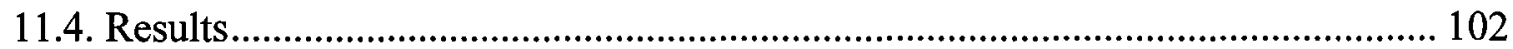

11.4.1. Purification and characterization of Wt and mutant yeast CBS. ................ 102

11.4.2. Purification and characterization of Wt and mutant human CBS................ 103

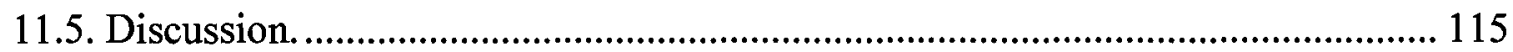

11.5.1. Characterization of F443A and D538A (hCBS)..................................... 115

11.5.2. Mutation of conserved glutamine residues impairs hCBS folding/activity. 116

11.5.3. Characterization of D444N and S466L (hCBS) ....................................... 117

11.5.4. Yeast CBS is SAM insensitive. ......................................................... 118

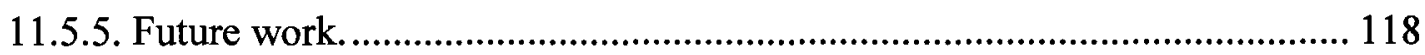

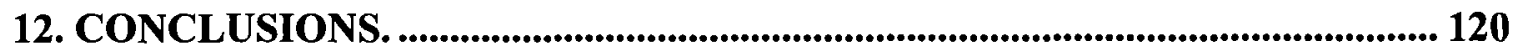

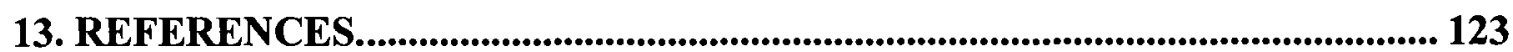




\section{LIST OF TABLES.}

Table 1. Comparison of the purification of hCBS expression constructs..........................56

Table 2. Comparison of the kinetic parameters of hCBS purified from the four aminoterminal, 6-His expression systems.......................................................................... 57

Table 3. Steady-state kinetic parameters of 6-His/hCBS and 6-His-linker/hCBS........... 58

Table 4. Steady-state kinetic parameters for ytCBS. ……….............................................. 84

Table 5. Primer sequences used for constructing $\mathrm{C}$-terminal domain mutants in yCBS

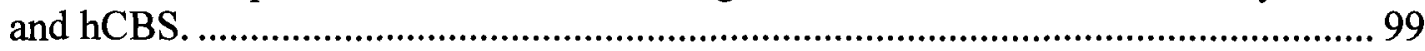

Table 6. Comparison of the kinetic parameters of yCBS-His wild-type and its C-terminal domain mutants.................................................................................................. 113

Table 7. Comparison of the kinetic parameters of his-hCBS and its $\mathrm{C}$ terminal domain mutants. 


\section{LIST OF FIGURES.}

Figure 1. The CBS-catalyzed condensation of L-Ser and L-Hcys forming L-Cth. [Aitken and Kirsch, 2005]

Figure 2. Schematic and cartoon representations of CBS structure. The domain structure of (A) human and (B) yeast CBS are similar, although the latter lacks the Nterminal, heme-binding domain [Jhee et al., 2000b]. (C) Cartoon and surface representation of the dimeric structure of the truncated hCBS enzyme [Meier et al., 2001; PDB ID: 1JBQ]. The PLP and heme cofactors are shown in yellow and red, respectively. One subunit of the dimer is shown in cartoon and the other in surface representation. The catalytic domain of each subunit is pale blue and the hemebinding domain, $\mathrm{N}$-terminal, is red.

Figure 3. The catalytic versatility of PLP is demonstrated by the breadth of reactions catalyzed by this cofactor. The first step of all PLP dependent reactions is the formation of an aldimine linkage with the amine group of the amino acid substrate. Thereafter, the reactions diverge as shown [Toney, 2005].

Figure 4. The chemical mechanism of the $\beta$-replacement reaction catalyzed by CBS. Stage I comprises the $\alpha, \beta$-elimination reaction in which the $\mathrm{C}_{\alpha}$ proton is abstracted and the hydroxyl group of the L-Ser side chain is eliminated, resulting in formation of the external aldimine of aminoacrylate. The aminoacrylate intermediate is subsequently attacked by the thiolate group of L-Hcys, producing L-Cth. The putative quinonoid intermediates are not shown, as they have not been observed for CBS [Miles et al., 2001].

Figure 5. View of the active site of hCBS [PDB: 1JBQ]. PLP (yellow) is shown in internal Schiff base linkage with lysine 119. The 5'-phosphate is anchored to the glycine 256 to the threonine 260 loop via several hydrogen bonds (not all are shown/indicated). Ser 349 is within hydrogen bonding distance to N-1 of the pyridine ring, and $\mathrm{O} 3$ ' of the pyridine ring is within hydrogen bonding distance to Asn 149. Residues S349 and N149, which correspond to S289 and N84 of yCBS are proposed to form hydrogen bonds (represented as solid lines) to the pyridine nitrogen and 3'-hydroxyl group of the PLP ring [Meier et al., 2001]. 18

Figure 6. The metabolism of homocysteine. Homocysteine is formed upon cleavage of $S$ adenosylhomocysteine (SAH), following demethylation of $S$-adenosylmethionine (SAM). Homocysteine can be remethylated, via the action of methionine synthase (MS), a vitamin $B_{12}$-dependent enzyme. In this reaction, methyltetrahydrofolate $\left(\mathrm{CH}_{3} \mathrm{THF}\right)$, which is formed from methylene tetrahydrofolate $\left(\mathrm{CH}_{2} \mathrm{THF}\right)$ through the action of methyltetrahydrofolate reductase (MTHFR), serves as the methyl donor. Alternatively, L-Hcys can be shunted down to the reverse transsulfuration pathway, where it is condensed with L-Ser, through the action of CBS, to form L-Cth, which is subsequently hydrolyzed by cystathionine $\gamma$-lyase (CGL), forming L-Cys, $\alpha$ ketobutyrate and ammonia. Additional enzymes in the remethylation pathway 
include MAT - Methionine adenyltransferase, MTs - Methyltransferases, and SAHH - $S$-adenosyl homocysteine hydrolase [Adapted from Hajjar, 2001].

Figure 7. Structural organization of CBS domains. (A) The cartoon representation of a generalized CBS domain, comprising two $\alpha$-helices and a $\beta$-sheet comprised of three strands [Bateman, 1997]. (B) generalized and (C) surface views of the CBS domain dimer of Streptococcus pyogenes IMPDH (PDB ID: 1ZFJ) [Zhang et al., 1999]. Helices are shown in dark grey and $\beta$-sheets are in light grey. The cleft marks the location of the dimer interface.

Figure 8. Binding interactions formed between adenine ligands and the CBS domains of CLC-5 (A) and AMPK (B). Coloring: $\alpha$-helices - dark grey; $\beta$ - sheets - light grey; loops - white; carbon, nitrogen, oxygen and phosphorus atoms - cyan, blue, red, and purple, respectively; H-bonds represented by dotted lines.

(A). CLC-5 in complex with ATP (PDB: 2J9L; Meyer et al., 2007). ATP stacking interaction of the adenine ring with Y617 of the CBS1 domain and hydrogen bonds between hydroxyl groups of the ribose moiety and D727 of the CBS2 domain. The corresponding residues are F439 (F443) and D501 (D538) in yCBS (hCBS), ........ 30

Residue T596 of CLC-5 forms a pair of hydrogen bonds with the 6'-amino group and the 1'-aza group of the adenine ring and S618 and K726 interact with the $\alpha$ - and $\gamma$ - phosphate groups, respectively, of the ATP ligand. The equivalent residues to S618 are D399 (D444) in yCBS (hCBS).

(B). Complex of AMPK with AMP (PDB ID: 2J9L; Day et al., 2007). Residue V225 of the CBS1 domain packs against the adenine ring, in place of the stacking interaction observed in the CLC-5-ATP complex (A), and hydrogen bonds are formed between S316 and the $\alpha$-phosphate and between D317 and hydroxyl groups of the ribose ring. The ribose sugar-binding motif in CBS2 stabilizes the hydroxyls of the ribose ring and the side chain of D317. The corresponding residues are F439 (F443) and D501 (D538) in yCBS (hCBS).

Residues A205 and A227 of AMPK form hydrogen bonds to the adenine ring of the AMP ligand and S226 interactions with the $\alpha$-phosphate. Residue S226 of AMPK corresponds to D399 (D444) in yCBS (hCBS).

Figure 9. Sequence alignment of CBS domains. Putative ribose binding motifs are shown in bold. Both pairs of SNF4 (the yeast analogue of AMPK) CBS domains (CBS1+2 and $\mathrm{CBS} 3+4$ ) are aligned with mouse CBS, rabbit CBS, rat CBS, yCBS, and hCBS. Residues involved in interactions with AMP in AMPK-1 (CBS3+4) are underlined and bolded. Residue 444 of CBS, which is substituted by asparagine (D444N) in homocystinuria, is shown in underlined italics. The hCBS peptide (511-531) protected by SAM from ${ }^{1} \mathrm{H} /{ }^{2} \mathrm{H}$-exchange is shown in bold italics [Sen et al., 2005]. 
Figure 10. Structures of SAM and ATP. Atoms numbers, symbols, and stereochemistry are shown in SAM.

Figure 11. Schematic representation of the five hCBS expression constructs: (A) 6-HisGST/hCBS, (B) 6-His-GFP/hCBS, (C) 6-His-linker/hCBS, (D) 6-His/hCBS, and (E) hCBS/6-His.

Figure 12. SDS-PAGE analysis of purified recombinant hCBS. Lanes: 1 and 9, protein molecular weight ladder (Benchmark, Invitrogen); 2, 6-His-GST/hCBS; 3, thrombin-treated 6-His-GST/hCBS; 4, 6-His-GFP/hCBS $(5 \mu \mathrm{g}) ; \mathbf{5}$, thrombin-treated 6-His-GFP/hCBS; 6, 6-His/hCBS; 7, 6-His-linker/hCBS; 8, 6-His-linker/hCBS treated with Factor $\mathrm{Xa}$. The $10 \%$ polyacrylamide gel was loaded with $5 \mu \mathrm{g}$ protein per lane and stained with Coomassie brilliant blue.

Figure 13. Steady-state initial velocity kinetics for hCBS showing the dependence of the reaction on [L-Hcys]. The concentration of L-Hcys was varied at fixed concentrations of L-Ser $(1.5 \mathrm{mM}, 0 ; 3.0 \mathrm{mM}, \square ; 6.0 \mathrm{mM}, \times ; 10 \mathrm{mM}, \Delta ; 20 \mathrm{mM}$, $\diamond ; 30 \mathrm{mM},+)$ and the fit of the entire data set to equation 2 is represented by the lines. The fitted parameters are given in Table 3 . Reactions were in a total volume of

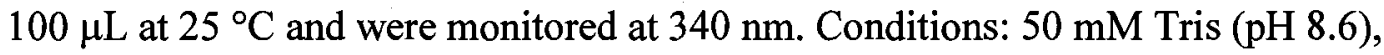
$1.5 \mathrm{mM}$ NADH, $20 \mu \mathrm{M}$ PLP, $1.6 \mu \mathrm{M} \mathrm{CBL}$, and $1.4 \mu \mathrm{M}$ LDH. Reactions were initiated by addition of $0.88 \mu \mathrm{M} \mathrm{hCBS}$.

Figure 14. Proposed mechanism of the $\alpha, \beta$-elimination half reaction of ytCBS demonstrating possible $E_{1}$ and $E_{2}$ reaction pathways, which are employed by $\operatorname{TrpS}$ and OASS, respectively [Jhee et al., 2001, Cook, 2003]. The labeled intermediates correspond to the (1) internal aldimine $(412 \mathrm{~nm}),(2)$ geminal diamine $(320-340 \mathrm{~nm})$, (3) external aldimine of L-Ser $(410-420 \mathrm{~nm}),(4)$ quinonoid $(\sim 480 \mathrm{~nm})$ and (5) external aldimine of aminoacrylate (320 and $460 \mathrm{~nm}$ ) [Jhee et al., 2001].

Figure 15. Dependence of the observed first-order rate constant $\left(k_{o b s}\right)$ for aminoacrylate formation, monitored at $460 \mathrm{~nm}$, on [L-Ser] for the S289A mutant. The S289A (16 $\mu \mathrm{M})$ mutant was incubated with $5-150 \mathrm{mM} \mathrm{L}-\mathrm{Ser}$ in $50 \mathrm{mM}$ Tris, $\mathrm{pH} 8.6$ at $25^{\circ} \mathrm{C}$. The line represents the fit of $k_{o b s} v s$. [L-Ser] fit to eq 6. Inset A: Spectra of $16 \mu \mathrm{M}$ S289A after $200 \mathrm{~s}$ reaction with 0 (solid line), 15, 50, 75, and $150 \mathrm{mM} \mathrm{L-Ser} \mathrm{(dotted}$ lines) show increasing intensity at 320 and $460 \mathrm{~nm}$, corresponding to the aminoacrylate intermediate, with increasing [L-Ser]. Inset B: Stability of the aminoacrylate intermediate of S289A. The S289A mutant $(16 \mu \mathrm{M})$ was equilibrated in $50 \mathrm{mM}$ Tris, $\mathrm{pH} 8.6$, for $15 \mathrm{~min}$ prior to addition of an equimolar concentration of L-Ser and the $460-\mathrm{nm}$ absorbance, corresponding to the aminoacrylate intermediate, was monitored over $360 \mathrm{~min}$. 85

Figure 16. Fluorescence spectra $\left(\lambda_{\mathrm{ex}}=298 \mathrm{~nm}\right.$ ) of S289A and (Inset) wild-type ytCBS alone (solid line) and in the presence of $50 \mathrm{mM} \mathrm{L}-\mathrm{Ser}$ (dashed line). Conditions: ytCBS or S289A $(20 \mu \mathrm{M})$ in $50 \mathrm{mM}$ Tris, pH 8.6. 
Figure 17. SDS-PAGE of 6-His-tagged yCBS and hCBS site-directed mutant enzymes.

(A) SDS-PAGE of fractions from purification of yCBS-D501A by Ni-NTA chromatography. Lane 6 and 17: protein marker; lane 1: cell lysate supernatant;

lane 2: column flow-through following loading; lane 3: column wash, lanes 4, 5, 716 and 18-20: every fifth protein fraction from the elution, starting with the first fraction. (B) SDS-PAGE of fractions from the purification of hCBS-D444N by NiNTA chromatography. Lane 5 and 14: protein marker; lane 1: cell lysate supernatant; lane 2: column flow-through following loading; lane 3: column wash; lanes 4, 5-13 and 15-17: every third fraction from the elution, starting from the first fraction. The $10 \%$ polyacrylamide gel was loaded with protein fractions per lane and stained with Coomassie brilliant blue. 105

Figure 18. Fluorescence-monitored denaturation of yCBS by 0-8 $\mathrm{M}$ urea. (A) The denaturation curves of yCBS $(\square)$, D399A $(\diamond)$, E417 ( $\times$ ), and D501A (O) yCBS samples were incubated overnight at $4^{\circ} \mathrm{C}$ overnight in $10 \mathrm{mM}$ Tris, $\mathrm{pH} 8.6$ containing 0-8 M urea. The other mutants, T414A, S416A, and T498A have similar protein unfolding equilibriums as the wild type and to maintain the pictures clarity, the data is not shown. The fluorescence intensities (f) at $330 \mathrm{~nm}\left(\lambda_{\mathrm{ex}}=280 \mathrm{~nm}\right)$ were normalized by equation 9 and plotted versus urea concentration. B. Emission spectra $\left(\lambda_{\mathrm{ex}}=280 \mathrm{~nm}\right)$ of $20 \mu \mathrm{M}$ yCBS-T414A showing the increasing peak at showing the increasing peak at $\sim 350 \mathrm{~nm}$ with increasing urea concentration $(0,2,2.25,2.5,2.75$, $3,3.25,3.5,4,4.5,5$, and $7 \mathrm{M})$.

Figure 19. Michaelis-Menten plots demonstrating the dependence of the reaction catalyzed by wild-type yCBS $(\diamond)$ and the D399A $(\square)$, and S416A $(\times)$ site-directed mutants on (A) varying [L-Ser] at a fixed L-Hcys concentration of $2 \mathrm{mM}$ and (B) varying [L-Hcys] at a fixed L-Ser concentration of $20 \mathrm{mM}$. The kinetic parameters are given in Table 6. Reactions conditions were $50 \mathrm{mM}$ Tris ( $\mathrm{pH} \mathrm{8.6),} 1.5 \mathrm{mM}$ NADH, $20 \mu \mathrm{M}$ PLP, $0.5 \mu \mathrm{M}$ CBL, $1.4 \mu \mathrm{M}$ LDH and $0.6-1.7 \mu \mathrm{M}$ wild-type or mutant yCBS.

Figure 20. Kinetics of hCBS proteins showing the dependence of the reaction on A. [LSer] with concentration of L-Hcys fixed at $2 \mathrm{mM}$ and B. [L-Hcys] with concentration of L-Ser fixed at $30 \mathrm{mM}$. ( $\diamond$ - hCBS; $\square-\mathrm{F} 443 \mathrm{~A}$; $\square$ - D538A). The fitted parameters are given in Table 7. Reactions conditions were $50 \mathrm{mM}$ Tris (pH 8.6), $1.5 \mathrm{mM}$ NADH, $20 \mu \mathrm{M}$ PLP, $1.6 \mu \mathrm{M}$ CBL, and $1.4 \mu \mathrm{M}$ LDH. Reactions were initiated by addition of $0.3-1.2 \mu \mathrm{M}$ hCBS 


\section{ABBREVIATIONS.}

\begin{tabular}{ll} 
Amp & Ampicilin \\
BSA & Bovine serum albumin \\
AA & Aminoacrylate \\
AMPK & AMP Protein Kinase \\
Asp AT & Aspartate aminotransferase \\
BSA & Bovine Serum Albumin \\
CBL & Cystathionine $\beta$-lyase \\
CGL & Cystathionine $\gamma$-lyase \\
CGS & Cystathionine $\gamma$-synthase \\
hCBS & Human Cystathionine $\beta$-synthase \\
ytCBS & truncated yeast Cystathionine $\beta$-synthase \\
yfCBS & Full-length Cystathionine $\beta$-synthase \\
CAPS & $N$-cyclohexyl-3-aminopropanesulfonic acid \\
CLC & Chloride channel protein \\
L-Cth & L-Cystathionine \\
DMSO & Dimethylsulfoxide \\
DTNB & 5,5 -Dithiobis-(2-nitrobenzoic acid) \\
DTT & Dithiolthretiol \\
E(A & internal aldimine intermediate \\
E(A ex) & external aldimine intermediate formed between the PLP \\
& cofactor and L-Ser \\
E(GD) & gem diamine species \\
E(AA) & $\alpha$-aminoacrylate external aldimine \\
E(Q) & quinonoid intermediate \\
EDTA & Ethylenediamine tetraacetic acid \\
ESB & External Schiff Base of the closed form of the enzyme \\
GFP & Green Fluorescent Protein \\
GST & Glutathione $S$-transferase \\
L-Hcys & L-Homocysteine \\
IMPDH & Inosine 5'-Monophosphate Dehydrogenase \\
IPTG & Isopropyl- $\beta-D$-thiogalactopyranoside \\
ISB & Internal Schiff Base of the free enzyme \\
ITC & Isothermal titration calorimetry \\
$\boldsymbol{K}_{\mathbf{d}(\text { app) }}$ L-Ser & The apparent dissociation constant for the E-AA complex due to L- \\
$\boldsymbol{K}_{\text {iF1 }}$ L-Hcys & Ser association with free enzyme. \\
$\boldsymbol{K}_{\text {iF2 }}$ L-Hcys & The E-Hcys dissociation constant; \\
& The dissociation constant for the E-Ser-Hcys complex due to L- \\
LDH & Hcys association with the E-Ser form of the enzyme before the \\
MES & aminoacrylate (AA) can be formed \\
MOE & Lactate Dehydrogenase \\
N-1 & 2-( $N$-morpholino)-ethanesulfonic acid \\
& Molecular Operating Environment \\
& Pyridine nitrogen in PLP \\
& \\
\hline
\end{tabular}




$\begin{array}{ll}\text { NADH } & \text { Nicotinamide Adenine Dinucleotide } \\ \text { OAS } & \text { O-Acetyl-L-Serine } \\ \text { OASS } & \text { O-Acetylserine Sulfhydrylase (A isozyme) } \\ \text { Ni-NTA } & \text { Ni-nitrilo triacetic acid } \\ \text { PLP } & \text { Pyridoxal 5'-phosphate } \\ \text { PMP } & \text { Pyridoxamine 5'-phosphate } \\ \text { SAH } & \text { S-adenosyl homocysteine } \\ \text { SAM } & \text { S-adenosyl methionine } \\ \text { L-Ser } & \text { L-Serine } \\ \text { trc } & \text { Hybrid trp-lac promoter } \\ \text { Tris } & \text { Tris-[hydroxymethyl]aminomethane } \\ \text { TS } & \text { Threonine Synthase } \\ \text { BTrpS } & \text { Beta subunit of Tryptophan Synthase }\end{array}$




\section{INTRODUCTION.}

Throughout history, diseases have had been a focus of social concern and superstition. Medical research has made great strides in eradicating, or reducing the prevalence of many diseases of historical significance, such as the bubonic plague, tuberculosis, leprosy and polio, particularly since the advent of antibiotics and vaccines. However, effective cures and/or therapeutics for many diseases, particularly those with a genetic component, such as cancer, Alzheimer's and Parkinson's, remain elusive.

Hereditary disorders can be divided into three groups: (1) mutations at the gene level, causing point or frameshift mutations in the protein encoded by the affected gene; (2) chromosomal abnormalities, resulting in a change in chromosome number and/or size; and (3) multi-factorial disorders, where mutations in multiple genes are often coupled with environmental factors. Phenylketonuria, sickle-cell anemia and homocystinuria are members of the first group, while Down syndrome and some forms of cancer are well-known examples of the second and third groups, respectively [Berg et al., 2002]. As our understanding of the genetic basis for many diseases and the availability of genetic testing have developed, screening of fetuses and newborn infants has increased. This is exemplified by phenylketonuria screening, which is widely available in North America and Europe [Shapiro, 1994]. However, as effective therapeutics are not currently available for many of the vast array of hereditary diseases, increased research in this area is necessary to understand their underlying mechanisms. 


\subsection{Homocystinuria.}

The plethora of the genetic disorders currently under investigation often seem to be vying for the spotlight, both in terms of public awareness and research funding. Homocystinuria is one such disorder and in recent years it has attracted attention due to its relationship with vascular disease. The elevated concentration of the amino acid homocysteine in the plasma, that is characteristic of homocystinuria, leads to the multi-systemic manifestations of this disorder, which involve many organs and tissues, notably the cardiovascular system, central nervous system and eyes [Kraus et al., 1999; McCully, 2001]. Homocysteine was discovered by Vincent Du Vigneuad in 1932, although its significance in clinical medicine was not initially appreciated and it was thought simply to be an obscure intermediate of amino acid metabolism [McCully, 2001]. Unlike methionine, its metabolic precursor, homocysteine is a non-proteinogenic amino acid. It was not until 1962, when a group of investigators in Belfast began to screen for amino acids in the urine of children with mental retardation, that the clinical relevance of homocysteine was recognized. Several children, presenting symptoms including dislocated ocular lenses (ectopia lentis), osteoporosis and skeletal problems, as well as vascular occlusions, were found to have homocysteine in their urine [McCully, 2001]. The disorder, which is the most common inborn error of methionine metabolism, was eventually named homocystinuria.

The most common cause of homocystinuria is deficiency of cystathionine $\beta$-synthase (CBS), the first enzyme of the reverse transsulfuration pathway [Kraus et al., 1999] The gene encoding CBS has also been linked to Down syndrome, as it is located on the $21^{\text {st }}$ chromosome in humans [Kamoun, 2001]. As is typical of a hereditary disorder, the incidence of homocystinuria is highly variable and dependent on ancestry. A frequency of $1: 344,000$ is estimated worldwide, but is much higher in specific populations [Mudd et al., 2001]. For 
example, incidences of 1:6,400, 1:65,000 and 1:20,500 have been recently reported in Norway, Ireland and Denmark, respectively, while a frequency of 1:349,000 has been estimated for the Czech Republic and Slovakia [Refsum et al., 2004; Yap and Naughten, 1998; Gallagher et al., 1995; Kozich et al., 1997; Janosik et al., 2001b]. Although small case studies have been conducted investigating the allele prevalence in Australia, Brazil, India, and the Middle East, statistical reports are not yet available for these populations [Maclean et al., 2002; Kaur et al., 1995; Porta et al., 2005; El-Said et al., 2006; Al-Essa et al., 1998].

More than 100 homocystinuria-associated mutations have been identified in the gene encoding CBS [Kraus et al., 1999]. Although several nonsense mutations, such as insertions, inversions and deletions, have been identified, $\sim 80 \%$ of homocystinuria-associated hCBS mutations are missense and result in a change in a single amino acid in the protein sequence. The majority of mutations are referred to as "private mutations" because they are either unique or restricted to only a few families. However, particular mutation(s) are also prevalent in specific populations. For example, G307S, one of the most common homocystinuria-associated mutations of CBS, is prevalent in patients of Celtic origin, particularly in Ireland where it accounts for $>70 \%$ of homocystinuria alleles tested [Kraus et al., 1999]. The prevalence of the I278T and G307S mutations has resulted in their becoming the standard mutations screened for in Northern European populations [Gaustadnes et al., 2000]. 


\subsection{Cystathionine $\beta$-synthase.}

Due to its position at the start of the reverse transsulfuration pathway, CBS plays an essential role in homocysteine metabolism. It catalyses the pyridoxal 5'-phosphate (PLP)dependant condensation of L-serine (L-Ser) and L-homocysteine (L-Hcys) to form L-cystathionine (L-Cth) (Figure 1), which is subsequently converted to L-cysteine (L-Cys), $\alpha$-ketobutyrate and ammonia by cystathionine $\gamma$-lyase (CGL) [Aitken and Kirsch, 2005; Kraus et al, 2002].

The PLP cofactor, required by both CBS and CGL, is common to enzymes catalyzing transformations of amino acids. Cystathionine $\beta$-synthase is typical of the phylogenetic group of PLP-dependent enzymes referred to as fold type II, which generally catalyze $\alpha, \beta$-replacement and elimination reactions of amino acid substrates [Christen and Mehta, 2001]. $O$-acetyl serine sulfhydrylase (OASS) is the prototypical representative of this group of enzymes and the mechanism of the Salmonella typhimurium enzyme has been thoroughly investigated [Christen and Mehta, 2001; Tai and Cook, 2001; Cook, 2003].

\subsubsection{CBS Structure.}

The strong homology between S. tymphirium OASS (stOASS) and the catalytic core of human CBS (hCBS) enabled the former to be used as a model for molecular replacement in the determination of the $2.6-\AA$ resolution crystal structure of the truncated, dimeric form of hCBS (Figure 2) [Meier et al., 2001]. The truncated form of hCBS comprises residues 1-413 and has a subunit molecular weight of $45 \mathrm{kDa}$, while the full-length enzyme is 551 amino acids in length, has a homotetrameric quaternary structure and a subunit molecular weight of $63 \mathrm{kDa}$. Each subunit of the full-length enzyme is comprised of three domains (Figure 3): the N-terminal domain ( $\sim 75$ residues); the highly-conserved catalytic core ( $\sim 340$ residues), which shares $47 \%$ 
sequence identity with stOASS; and the C-terminal, regulatory domain ( 140 residues), which contains the proposed binding site of the allosteric activator molecule, $S$-adenosylmethionine (SAM) [Meier et al., 2001; Bateman, A., 1997]. Full-length hCBS is prone to aggregation, which has hindered biophysical studies, thereby necessitating the removal of the C-terminal 138 residues to enable the production of crystals of this enzyme [Kraus et al., 1998; Kraus et al., 2000; Meier et al., 2001]. Therefore, no structure is available for the regulatory domain of CBS.

The PLP cofactor of hCBS is covalently bound, via a Schiff-base linkage, to K119 in the active site of the enzyme. Each subunit of hCBS also possesses a heme moiety, which is situated in the N-terminal domain (Figure 2), where it is axially coordinated by residues $\mathrm{C} 43$ and $\mathrm{H} 65$ [Meier et al., 2001]. The presence of the heme-binding, $\mathrm{N}$-terminal domain is uniquely associated with mammalian CBS (Figure 3) [Kraus et al., 1996]. The role of the heme cofactor of hCBS has yet to be elucidated, although the possibility of a redox-regulatory mechanism is attractive as it could allow for regulation of the flux through the reverse transsulfuration pathway, which produces the L-Cys required for the biosynthesis of glutathione, a key compound in the maintenance of cellular redox stability [Jhee et al., 2000a; McLean et al., 2000; Burstyn et al., 2004]. 
Figure 1. The CBS-catalyzed condensation of L-Ser and L-Hcys forming L-Cth. [Aitken and Kirsch, 2005] 


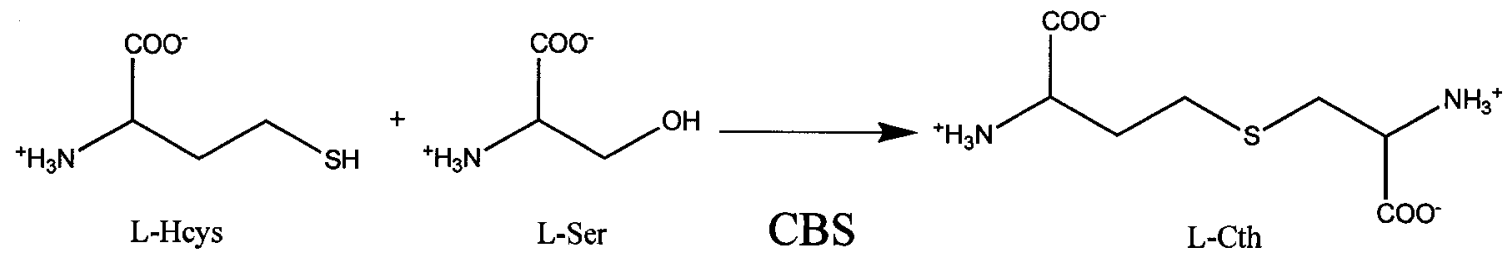


Figure 2. Schematic and cartoon representations of CBS structure. The domain structure of (A) human and (B) yeast CBS are similar, although the latter lacks the N-terminal, heme-binding domain [Jhee et al., 2000b]. (C) Cartoon and surface representation of the dimeric structure of the truncated hCBS enzyme [Meier et al., 2001; PDB ID: 1JBQ]. The PLP and heme cofactors are shown in yellow and red, respectively. One subunit of the dimer is shown in cartoon and the other in surface representation. The catalytic domain of each subunit is pale blue and the hemebinding domain, $\mathrm{N}$-terminal, is red. 
A.
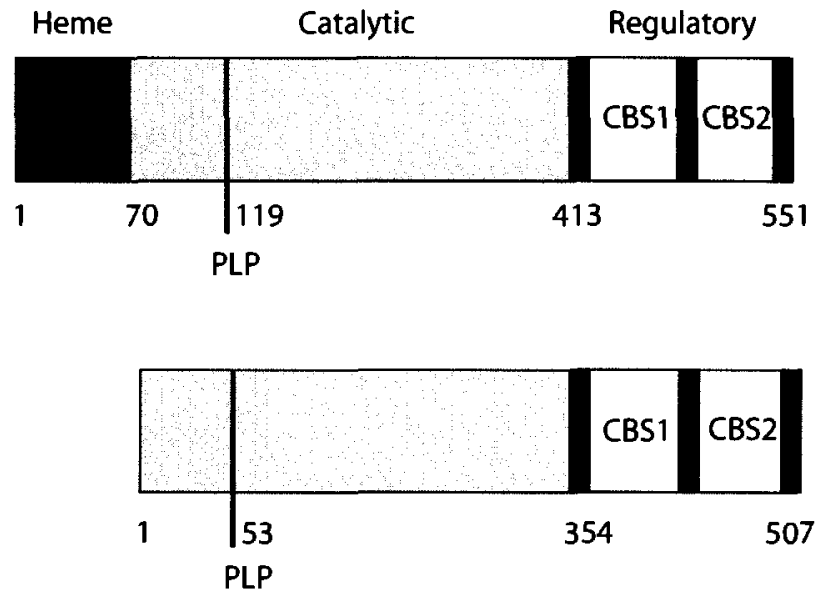

B.

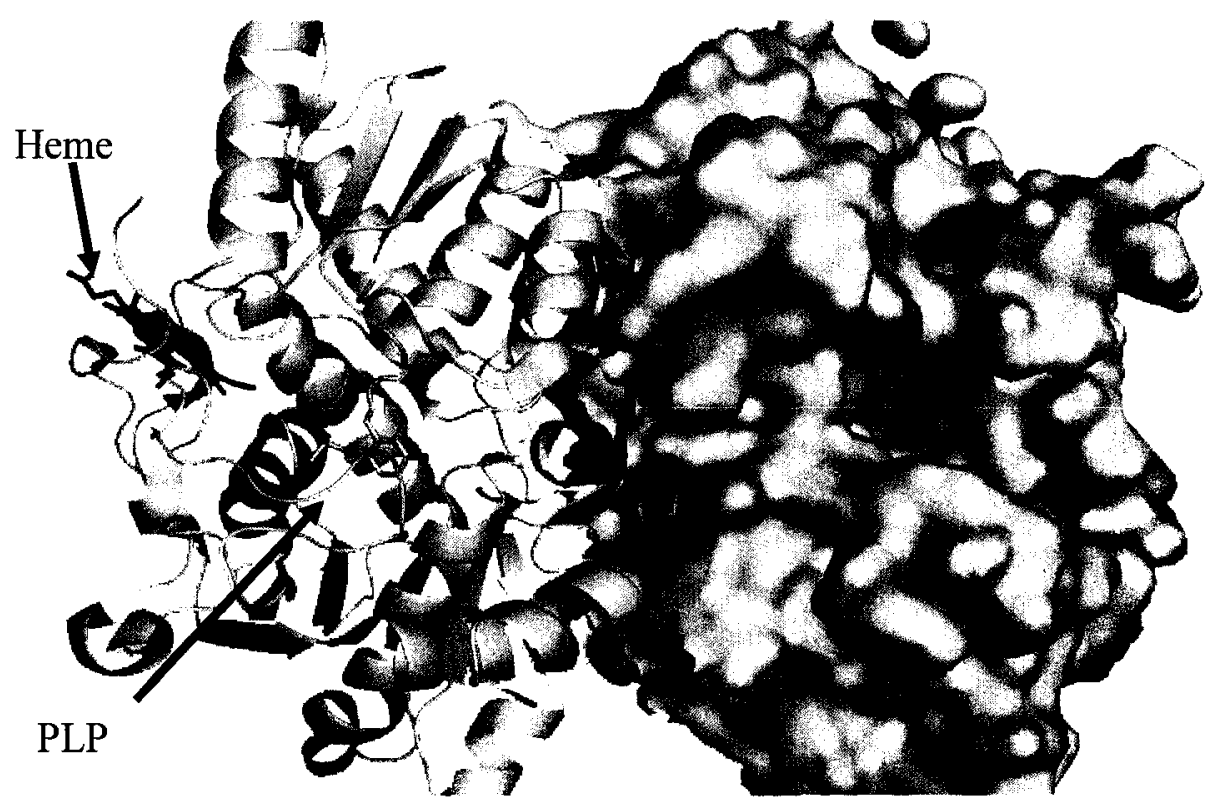




\subsubsection{Mechanism of $C B S$.}

The ping-pong kinetic mechanism of CBS, in which L-Ser binding and release of $\mathrm{H}_{2} \mathrm{O}$ precedes L-Hcys binding and the release of L-Cth, has been established [Jhee et al, 2000b; Banerjee \& Zou, 2005]. However, the mechanism whereby this enzyme regulates the chemistry of the versatile PLP cofactor has not been fully elucidated. Pyridoxal 5'-phosphate-dependant enzymes represent approximately $4 \%$ of those recognized by the Enzyme Commission [Christen and Mehta, 2001]. The PLP cofactor is derived from pyridoxine, also known as Vitamin $\mathrm{B}_{6}$ [Mozzarelli and Betati, 2006]. Oxidation of pyridoxine to the aldehyde form (pyridoxal) and phosphorylation at the $5^{\prime}$ position is required to produce the activated PLP form of the cofactor [Toney, 2004]. Pyridoxal 5'-phosphate-dependent enzymes carry out a diverse array of reactions, including transamination, decarboxylation, racemization, and side chain rearrangements of amino acid substrates (Figure 3) [Christen and Mehta, 2001].

It is the electron-withdrawing capacity of the pyridinium ring of the PLP cofactor that enables cleavage of the bond between $\mathrm{C}_{\alpha}$ and either the $\mathrm{C}_{\alpha}$-proton, side chain or $\alpha$-carboxyl group of amino acid substrates (Figure 4) as well as the stabilization of the ensuing carbanion. The reaction specificity of PLP-dependent enzymes is provided by the protein component of the enzyme, which controls the substrate specificity, modifies the electron-withdrawing nature of the pyridinium ring and determines the orientation of both the cofactor and the substrate in the active site [Eliot and Kirsch, 2004]. The effect of regulating substrate orientation with respect to the aromatic ring of the cofactor is well understood, as it is the bond to the $\mathrm{C}_{\alpha}$ substituent, of the amino acid substrate, in the position orthogonal to the pyridinium ring that is cleaved to generate a carbanion during catalysis [Dunathan, 1966]. 
Figure 3. The catalytic versatility of PLP is demonstrated by the breadth of reactions catalyzed by this cofactor. The first step of all PLP dependent reactions is the formation of an aldimine linkage with the amine group of the amino acid substrate. Thereafter, the reactions diverge as shown [Toney, 2005]. 


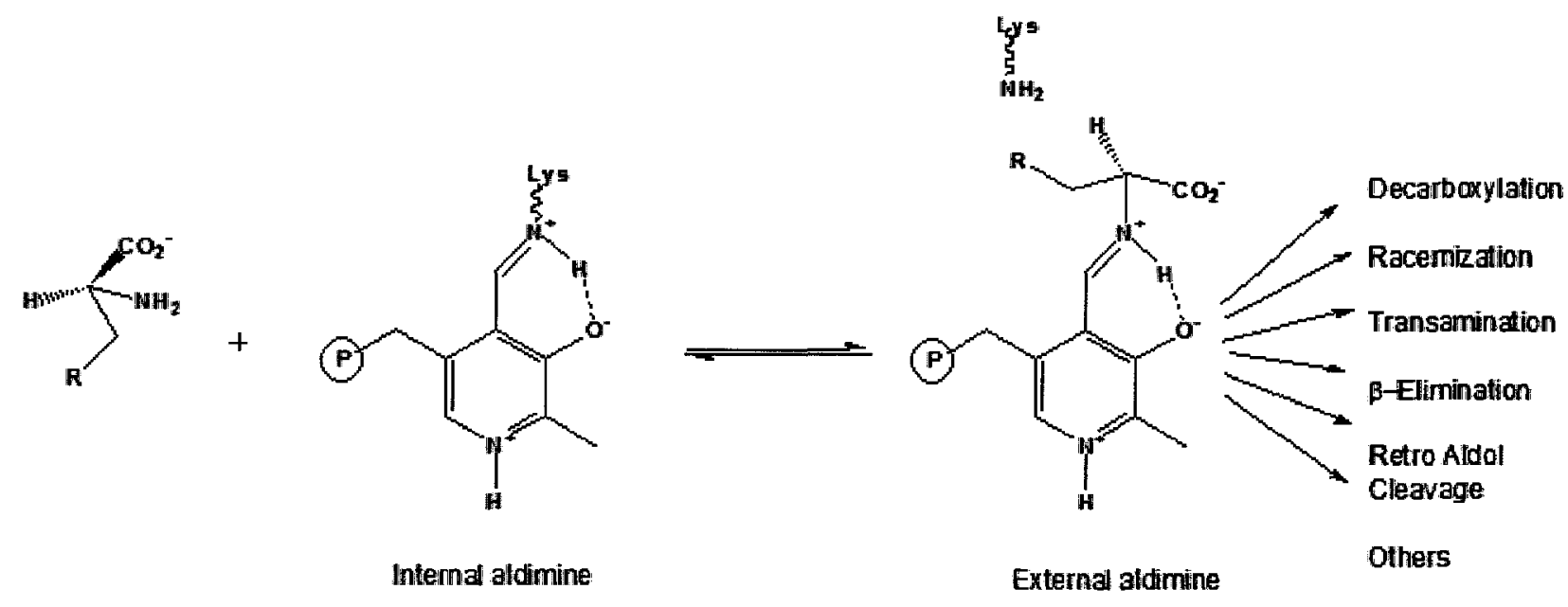


Other effects regulating reaction specificity, such as the nature of the active-site residues interacting with the ring substituents of the cofactor, are more subtle and remain to be defined for many PLP-dependent enzymes, including CBS.

The first step in catalysis, common to all PLP-dependent enzymes catalyzing transformations of amino acid substrates, is a Schiff-base exchange reaction referred to as transimination. The resting state of a PLP-dependent enzyme is the internal aldimine, in which the cofactor is covalently bound, as a Schiff base, to the side chain of an active-site lysine residue (K119 of hCBS, Figure 2). The $\varepsilon$-amino group of the active-site lysine is displaced by the amine group of the substrate, resulting in the formation of an aldimine linkage with the substrate, referred to as the external aldimine (Eliot and Kirsch, 2004). This reaction involves the formation of a geminal diamine intermediate and occurs via a multi-step process that is generally very rapid in comparison with the central steps in the reaction mechanism (e.g., deprotonation of $\mathrm{C}_{\alpha}$ ). The external aldimine is the common central intermediate for all enzymatic and nonenzymatic PLPcatalyzed reactions and divergence in reaction specificity occurs from this point (Figure 4).

Reactions catalyzed by PLP-dependent enzymes occur via a number of steps that generally involve the flow of electrons between the cofactor and the covalently-bound substrate. As the extent of conjugation of the aromatic system of the cofactor is altered as the reaction proceeds (i.e. by protonation and deprotonation of specific positions), PLP intermediates having distinct spectral properties are observed. This property of PLP facilitates pre-steady state investigations of enzymes dependent on this versatile cofactor. However, hCBS is exceptional in this regard, as the Soret band of the heme cofactor masks the weaker absorbance of the PLP intermediates. Since yeast CBS (yCBS) lacks the N-terminal, heme-binding domain that is found in CBS from mammalian sources, and is expressed in $>50$-fold greater quantities than the human 
enzyme, it has proven a useful model for mechanistic studies (Bukovska et al., 1994; Shan and Kruger, 1998; Jhee et al., 2000a; Aitken and Kirsch, 2003, Aitken and Kirsch, 2004).

The reaction catalyzed by CBS is typical of PLP-dependent, $\beta$-replacement reactions in that it is comprised of two distinct stages [Miles et al., 2001] In stage I, a transimination reaction, occurring via a geminal diamine intermediate, results in the formation of the external aldimine of L-Ser (Figure 4). Subsequently, the $\mathrm{C}_{\alpha}$ proton of L-Ser is abstracted and the hydroxyl group of LSer undergoes $\beta$-elimination, resulting in the formation of the external aldimine of aminoacrylate (E-AA). Stage II commences with a nucleophilic attack on the aminoacrylate intermediate by the thiolate group of L-Hcys, followed by reprotonation at $\mathrm{C}_{\alpha}$ to generate the external aldimine of $\mathrm{L}-$ Cth. A final transimination reaction releases the product, L-Cth, regenerating the internal aldimine form of the enzyme [Jhee et al., 2000a; Jhee et al., 2001; Miles et al., 2001; Banerjee and Zou, 2005]. Similar reaction mechanisms have been established for OASS and tryptophan synthase (TrpS), which are also members of fold-type II of PLP enzymes [Miles et al., 1998; Cook, 2003; Mozzarelli and Bettati, 2006; Christen and Mehta, 2001].

The particular PLP intermediates formed during the course of a reaction is an important determinant of reaction specificity that is regulated in-part by interaction between ring substituents and neighboring active-site residues. Investigations of aspartate amino transferase, the archetypical PLP-dependent enzyme, have demonstrated that the nature of interactions with the pyridinium nitrogen, at position $\mathrm{N}-1$, and the hydroxyl group attached to $\mathrm{C} 3$ of the PLP ring, are of particular importance in this regard [Goldberg et al., 1991; Yano et al., 1992]. The residues interacting with the pyridinium nitrogen and the hydroxyl ring substituent in hCBS are S349 and N149, respectively (Figure 5), which correspond to S289 and N84 of yCBS (Meier $e t$ al., 2001). 
$\alpha, \beta$-elimination reactions may proceed, dependent on the leaving group in the $\beta$-position, via an $\mathrm{E}_{1}$ or $\mathrm{E}_{2}$ chemical mechanism. Pyridoxal 5'-phosphate-dependent $\alpha, \beta$-elimination reactions are generally thought to occur via an $\mathrm{E}_{1}$ mechanism in which the carbanion formed upon abstraction of the $\mathrm{C}_{\alpha}$ proton is stabilized by resonance delocalization in the quinonoid intermediate, which would not be formed in a concerted, $\mathrm{E}_{2}$ reaction [Mayer et al., 1984; Tai and Cook, 2001]. A quinonoid intermediate, the formation of which requires either protonation of or formation of a hydrogen bond to N-1 of the PLP cofactor, is observed in both stages of the Salmonella typhimurium $\operatorname{TrpS}$ (stTrpS) reaction (Jhee et al., 1998a). In st TrpS, it is the serine residue at position 377 (S377) that forms a hydrogen bond to $\mathrm{N}-1$ of the cofactor, thereby enabling formation of the quinonoid intermediate in this enzyme. The S377D site-directed mutant of TrpS displays a prominent band around $500 \mathrm{~nm}$, typical of a quinonoid, in the presence of the L-Ser substrate, that is not observed in equilibrium studies with the wild-type enzyme, demonstrating that the presence of a carboxylate group in proximity to N-1 of the PLP cofactor forces protonation of the pyridinium nitrogen and stabilizes the quinonoid intermediate to an greater extent than the wild-type enzyme [Jhee et al., 1998a]. In contrast, no quinonoid intermediate has been detected in pre-steady state studies of OASS, which follows an $E_{2}$ mechanism, or CBS [Tai and Cook, 2001; Jhee et al., 2001]. 
Figure 4. The chemical mechanism of the $\beta$-replacement reaction catalyzed by CBS. Stage I comprises the $\alpha, \beta$-elimination reaction in which the $C_{\alpha}$ proton is abstracted and the hydroxyl group of the L-Ser side chain is eliminated, resulting in formation of the external aldimine of aminoacrylate. The aminoacrylate intermediate is subsequently attacked by the thiolate group of L-Hcys, producing L-Cth. The putative quinonoid intermediates are not shown, as they have not been observed for CBS [Miles et al., 2001]. 
Stage I

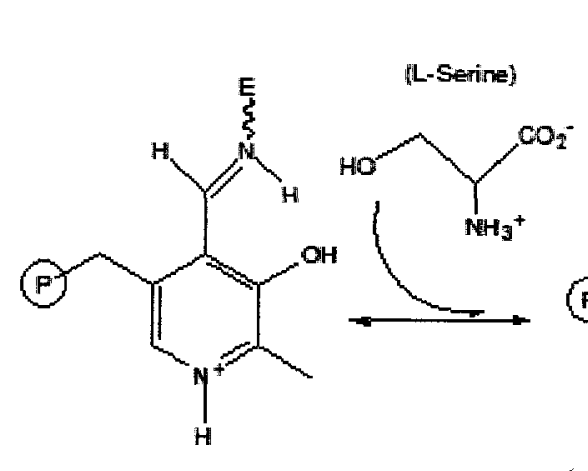

E

$412 \mathrm{~nm}$<smiles>CCNC(N[C@@H](C)CO)c1c(CBr)c[nH+]c(C)c1[O-]</smiles>

E-GD-I

$320-340 \mathrm{~nm}$

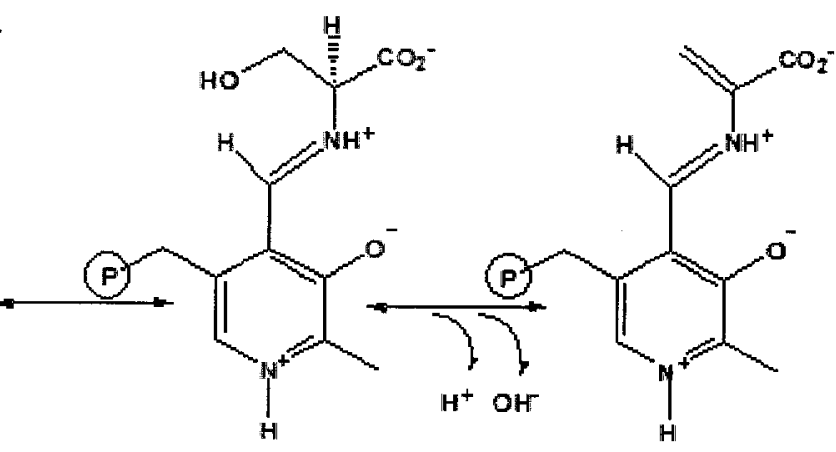

E-Ser 410-420 nm
E.AA

$460 \mathrm{~nm}$

$320 \mathrm{~nm}$

Stage II

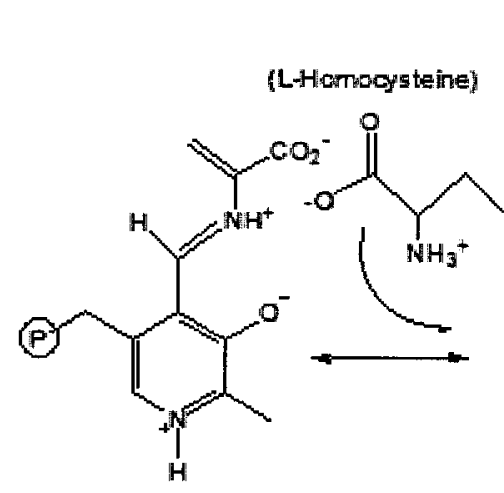

E-AA

$460 \mathrm{~nm}$ $320 \mathrm{~nm}$<smiles>CSOC(=O)C([NH3+])CSCC(/N=C/c1c(CBr)cnc(C)c1[O-])C(=O)O</smiles>

E-Cyst $420 \mathrm{~nm}$

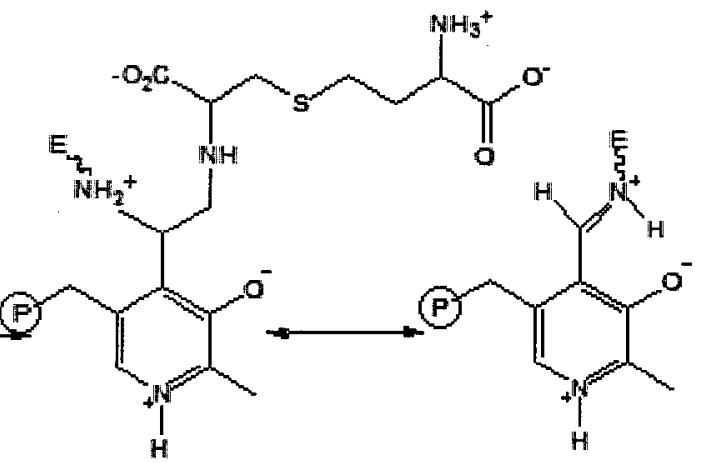

E-GDII 320-340 nm
E 
Figure 5. View of the active site of hCBS [PDB: 1JBQ]. PLP (yellow) is shown in internal Schiff base linkage with lysine 119. The 5'-phosphate is anchored to the glycine 256 to the threonine 260 loop via several hydrogen bonds (not all are shown/indicated). Ser 349 is within hydrogen bonding distance to $\mathrm{N}-1$ of the pyridine ring, and $\mathrm{O} 3$ ' of the pyridine ring is within hydrogen bonding distance to Asn 149. Residues S349 and N149, which correspond to S289 and N84 of yCBS are proposed to form hydrogen bonds (represented as solid lines) to the pyridine nitrogen and 3'-hydroxyl group of the PLP ring [Meier et al., 2001]. 


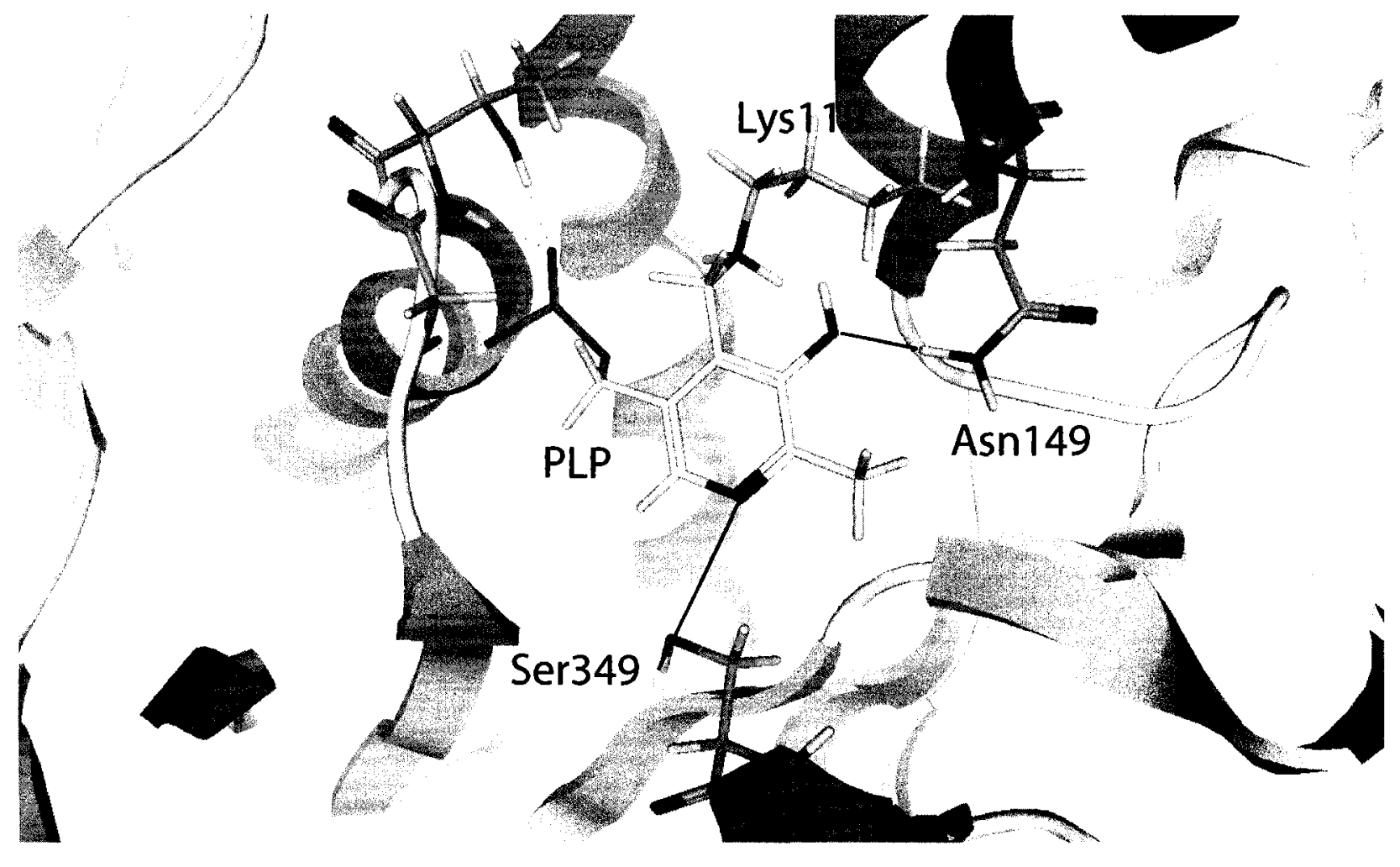


Tai and Cook have reasoned that formation of a quinonoid intermediate is disfavored in the reaction catalyzed by OASS because the $\mathrm{p} K_{a}$ 's of the pyridine nitrogen of the PLP and the adjacent S272 are unmatched, making unlikely that S272 unlikely to donate a proton to N1 of the cofactor [Tai et al., 2003]. Alignment of the sequences of yCBS (or hCBS) with stOASS and stTrpS demonstrate that while S289 of yCBS corresponds to S272 of stOASS, the corresponding residue of stTrpS is a glutamate. Although S377 of stTrpS interacts with the N-1 of the PLP ring, this residue is not structurally equivalent to S272 of stOASS and S289 of yCBS. Therefore, the role of S289 of yCBS is of particular interest because while this enzyme is more closely related structurally to stOASS, which employs an $E_{2}$ reaction mechanism that does not involve the formation of a quinonoid intermediate, the first stage of yCBS catalysis is identical to that of stTrpS, which employs an $E_{1}$ mechanism [Cook, 2003]. The role of S289 in the regulation of cofactor chemistry in yCBS will be investigated in this study.

\subsubsection{Regulation of CBS activity.}

Homocysteine is formed in vivo upon cleavage of $S$-adenosylhomocysteine (SAH), which is produced following loss of the methyl group of $S$-adenosylmethionine (SAM), a key methyl donor in cellular metabolism and precursor of a variety of compounds, including nucleotides. The transmethylation and reverse transsulfuration pathways compete for the branch-point metabolite L-Hcys, converting it to L-Met, the immediate precursor of SAM, and L-Cys,

respectively (Figure 6) [Selhub, 1999; Skovby et al., 1995]. The relative flux through these two pathways is roughly equal and is controlled, via allosteric regulation, by SAM [Kraus et al., 1994]. Allosteric activation of hCBS by SAM increases the flux of L-Hcys through the reverse transsulfuration pathway when the cellular methionine pool exceeds the level required to 
maintain homeostasis, a concentration that may vary depending upon the metabolic state of the cell [Finkelstein et al., 1975; Kraus et al., 2001]. Human CBS is activated 2- to 3-fold by SAM, which is proposed to bind to an, as-yet uncharacterized, site within the regulatory domain [Finkelstein et al., 1975].

The C-terminal regulatory domain of CBS contains two hydrophobic sub-domains, referred to as CBS1 and CBS2 (Figure 2). This motif, known as a CBS domain because it was first observed in CBS, is also found in other proteins, including inosine-5'-monophosphate dehydrogenase (IMPDH) and AMP protein kinase (AMPK), in which it has been demonstrated to contain a binding site for adenosine-containing ligands, such as SAM and adenosine triphosphate (ATP) [Bateman, 1997; Ignoul and Eggermount, 2005]. Proteolytic cleavage, expression of the truncated enzyme form, thermal activation and specific homocystinuriaassociated mutations have also been observed to result in an elevation of hCBS activity [Banerjee et al., 2005]. These findings demonstrate that it may be possible to develop drugs that interact with the $\mathrm{C}$-terminal domain of CBS to treat elevated homocysteine in humans.

The yeast and human CBS enzymes have similar domain architectures, although the yeast enzyme lacks the N-terminal, heme-binding domain of hCBS. However, while the yeast enzyme, unlike hCBS, is reported to be unresponsive to SAM, removal of the regulatory domain of both $\mathrm{hCBS}$ and yeast CBS (yCBS) results in a truncated, dimeric form of the enzyme that is activated $\sim 3$-fold compared to the full-length tetrameric form [Jhee et al., 2000b]. Therefore, as no allosteric activator of $\mathrm{yCBS}$ has been reported, the role of the regulatory domain within the context of this enzyme is unclear. 
Figure 6. The metabolism of homocysteine. Homocysteine is formed upon cleavage of $S$ adenosylhomocysteine (SAH), following demethylation of $S$-adenosylmethionine (SAM). Homocysteine can be remethylated, via the action of methionine synthase (MS), a vitamin $B_{12-}$ dependent enzyme. In this reaction, methyltetrahydrofolate $\left(\mathrm{CH}_{3} \mathrm{THF}\right)$, which is formed from methylene tetrahydrofolate $\left(\mathrm{CH}_{2} \mathrm{THF}\right)$ through the action of methyltetrahydrofolate reductase (MTHFR), serves as the methyl donor. Alternatively, L-Hcys can be shunted down to the reverse transsulfuration pathway, where it is condensed with L-Ser, through the action of CBS, to form $\mathrm{L}$-Cth, which is subsequently hydrolyzed by cystathionine $\gamma$-lyase (CGL), forming L-Cys, $\alpha$ ketobutyrate and ammonia. Additional enzymes in the remethylation pathway include MAT Methionine adenyltransferase, MTs - Methyltransferases, and SAHH - S-adenosyl homocysteine hydrolase [Adapted from Hajjar, 2001]. 


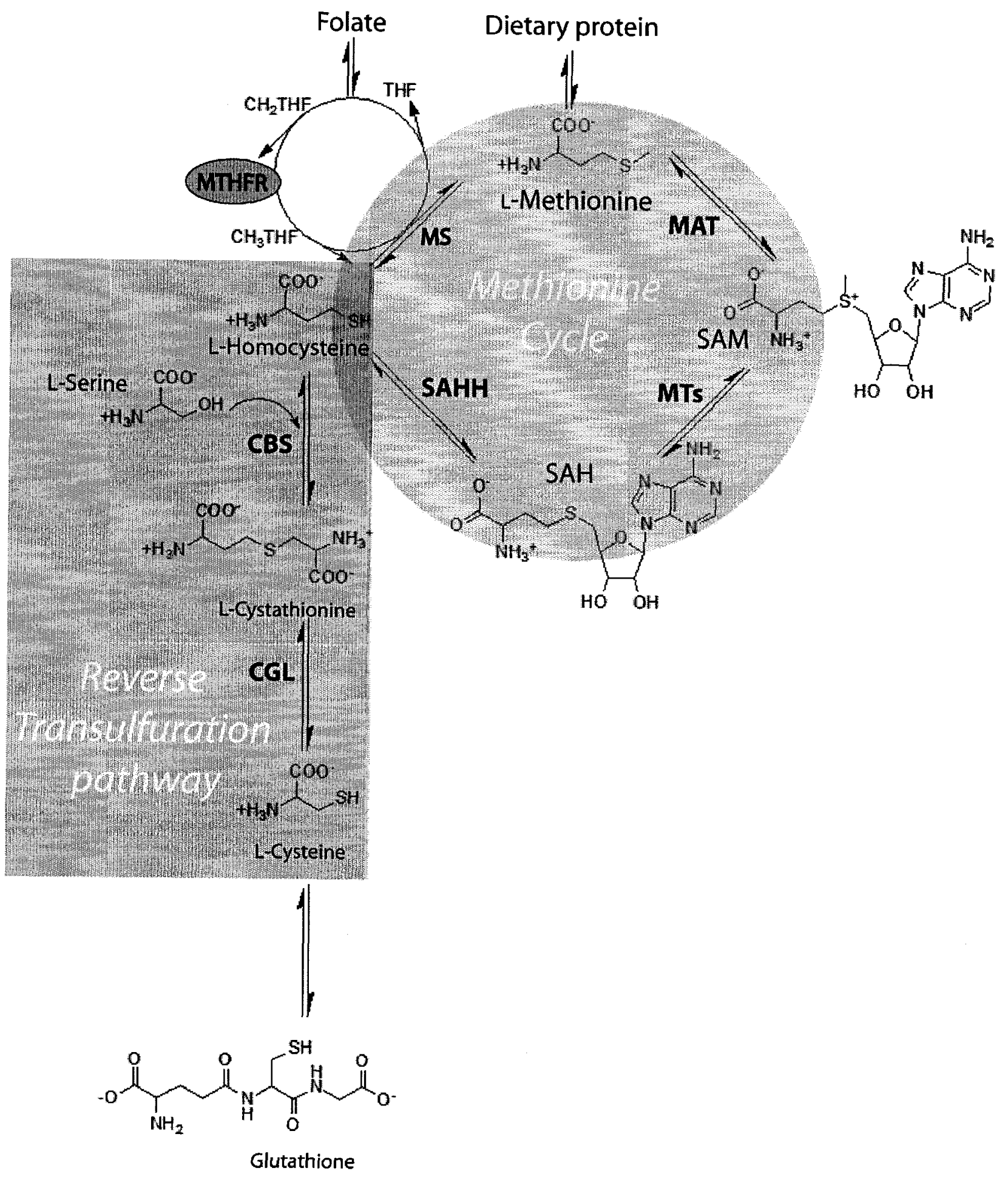




\subsection{CBS domains.}

CBS domains have been found in proteins from archaebacterial, eubacterial and eukaryotic sources [Bateman, A., 1997]. Point mutations in the CBS domains of a range of proteins are linked to hereditary diseases: IMPDH with retinitis pigmentosa, CBS with homocystiniuria, AMPK with familial hypertrophic cardiomyopathy and Wolff-Parkinson-White syndrome, and the different isoforms of chloride-channel protein (CLC-2, CLC-5, CLC-6) with various disorders including Dent's Disease, congenital myotonia, idiopathic generalized epilepsy, hypercalciuric nephrolithiasis [Kennan et al., 2002; Nozaki et al., 2001; Blair et al., 2001; Maduke et al., 1999]. Although their specific role likely varies with the nature of the protein each is a component of, involvement in oligomerization and the binding of adenosinecontaining ligands are common properties of CBS domains [Ignoul and Eggermont, 2005; Scott et al., 2004]. Their role as determinants of the oligomeric status of CBS is demonstrated by the observation that removal of the 141 or 154 residues comprising the C-terminal, regulatory domains of human and yeast CBS, respectively, converts the quaternary structure of these enzymes from homotetrameric to homodimeric [Kery et al., 1998; Miles et al., 2000].

CBS domains are comprised of a conserved $\beta_{1}-\alpha_{1}-\beta_{2}-\beta_{3}-\alpha_{2}$ motif and generally occur in pairs, as observed in both human and yeast CBS, CLC proteins and IMPDH, or double pairs, as exemplified by AMPK and its yeast homolog, Snf4. Structures of CBS domains from IMPDH, AMPK and Snf4 demonstrate that dimerization of CBS domain pairs results in the formation of a symmetrical unit [Zhang et al., 1999; Tong et al., 2006; Hardie et al., 1998]. Although the two amphipathic $\alpha$ helices of each monomer contribute to the inter-domain surface, dimer formation is largely driven by interactions between the $\beta$-sheets of the two domains, resulting in the formation of a characteristic cleft at the interface (Figure 7). Several reports indicate that the 
regulatory domain is the site of SAM binding in hCBS [Kraus et al., 1998, Kruger et al., 2001; Kraus et al., 2001; Banerjee et al., 2002]. For example, deletion of the $145 \mathrm{C}$-terminal amino acids of CBS abolishes activation of the enzyme by SAM [Kraus et al., 2004]. A selection of homocystinuria-associated mutations resulting in insensitivity to SAM binding have also been identified. These mutations, including C431A and I435T, D444N and S466L (corresponding to V382, I390, D399 and R421 of yCBS), are situated within the regulatory domain and may provide clues to the location of the binding site of SAM or the mechanism of allosteric activation [Kluijtmans et al., 1996; Kruger and Cox, 1995; Shan et al., 2001]. The S466L and I435T mutant enzymes are constitutively activated and bind SAM without further activation [Janosik et al., 2001b], suggesting that these residues may not be directly involved in SAM binding. In contrast, biophysical characterization of the D444N mutant revealed that although it is conformationally locked in an activated state, its affinity for SAM is reduced by 15 -fold [Hardle et al., 2004; Banerjee et al., 2005]. Similarly the C431S mutant is constitutively activated, but does not bind SAM [Kraus et al., 2006], suggesting the possibility that this residue may be involved in a disulfide bridge that maintains the appropriate structure of the SAM-binding site. Another clue to the location of the SAM binding site was provided by an experiment employing ${ }^{1} \mathrm{H} /{ }^{2} \mathrm{H}$-exchange and peptide mass mapping to identify residues protected from exchange upon SAM binding. A single peptide, extending from residues 511-531, was identified as being sensitive to the presence of SAM [Banerjee et al., 2005]. 
Figure 7. Structural organization of CBS domains. (A) The cartoon representation of a generalized CBS domain, comprising two $\alpha$-helices and a $\beta$-sheet comprised of three strands [Bateman, 1997]. (B) generalized and (C) surface views of the CBS domain dimer of

Streptococcus pyogenes IMPDH (PDB ID: 1ZFJ) [Zhang et al., 1999]. Helices are shown in dark grey and $\beta$-sheets are in light grey. The cleft marks the location of the dimer interface. 
A.

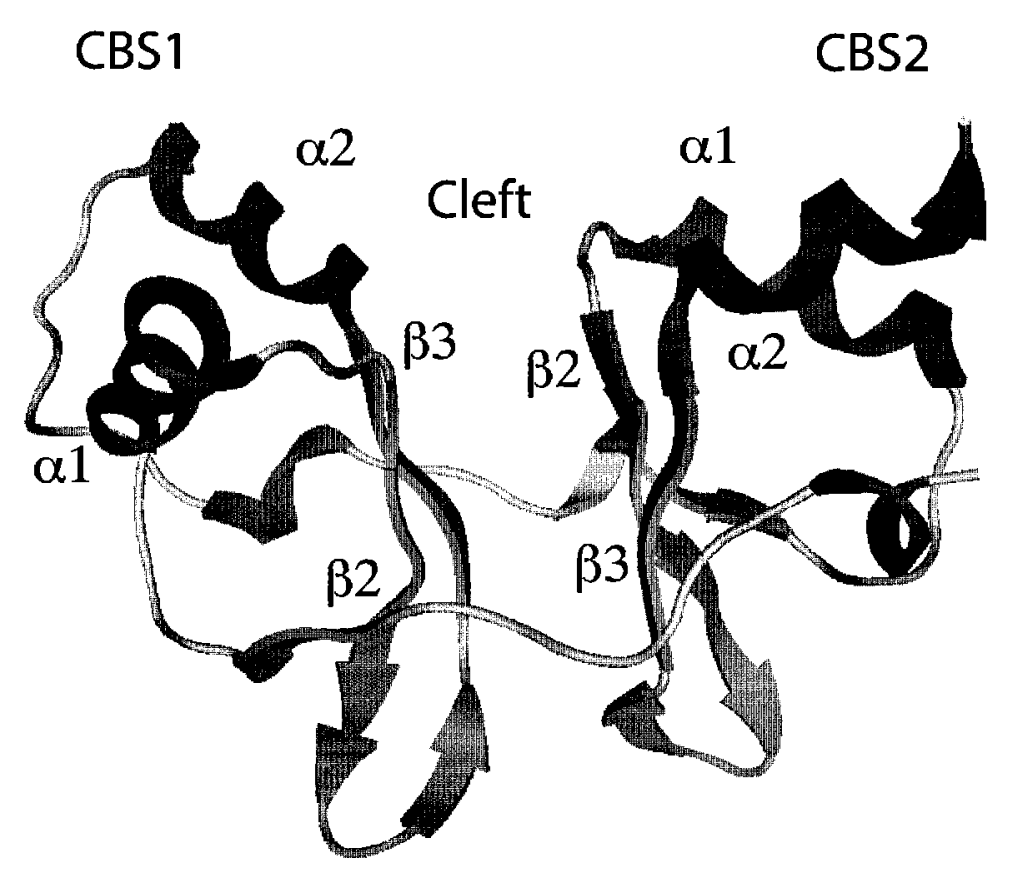

B.

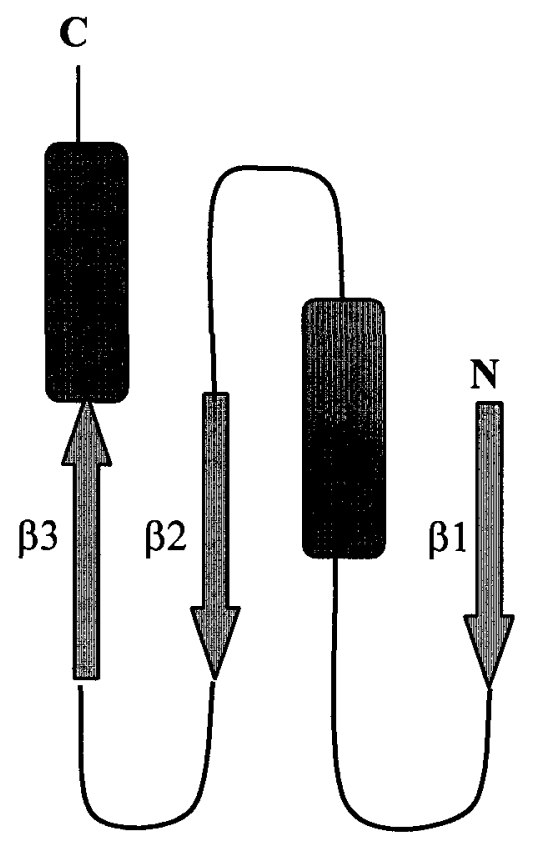

C.

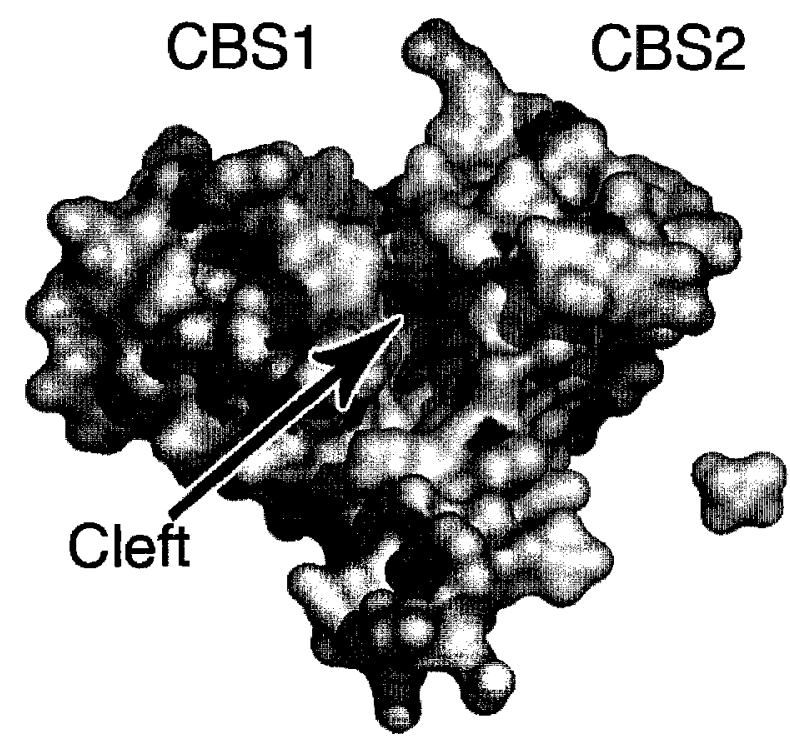




\subsubsection{Nucleotide binding in other CBS domain proteins.}

The tendency of full-length CBS to aggregate has hindered structural investigations of this enzyme, necessitating removal of the regulatory domain to solve the crystal structure of residues 1-413 of hCBS [Meier et al., 2001; Kraus et al., 2000]. Therefore, detailed structural information is not available for the C-terminal regulatory domain and clues to its structural organization, the location of the allosteric site and the role of selected amino acids in the allosteric activation and oligomerization of the enzyme must be obtained from biochemical observations and modeling studies based on the structures of CBS domains in other proteins.

\subsubsection{Inosine-5'-monophosphate dehydrogenase.}

IMPDH is a key enzyme in the de novo synthesis of guanine nucleotide biosynthesis [Morris et al., 1975]. The native enzyme is a homotetramer, the core of which is formed by the interaction of the catalytic domain of each subunit, while the four pairs of CBS domains are situated at the periphery and are approximately $35 \AA$ from the active site [Zhang et al., 1999]. Binding of ATP, the allosteric activator of IMPDH, results in increased cooperativity of substrate binding, which is eliminated by the naturally-occurring R224P mutation found in retinitis pigmentosa patients [Scott et al., 2004; Bowne et al., 2002].

\subsubsection{Chloride-channel protein.}

Chloride channels $(\mathrm{ClC})$ are membrane proteins and are involved in a wide variety of cellular functions including membrane excitation potentials, cell volume regulation and cell proliferation [Jentsch et al., 1990; Jentsch et al., 1996; Maduke et al., 2000; Jentsch et al., 1999]. All mammalian ClC proteins possess cytoplasmic CBS domains [Ponting, 1997]. Their role in 
$\mathrm{ClC}$ channel function remains unresolved, although several reports indicate that $\mathrm{CBS}$ domains may be involved in the regulation of protein function by intracellular nucleotides like ATP, ADP, and AMP [Scott et al., 2004; Bennetts et al., 2005; Wellhauser et al., 2006; Meyer et al., 2007]. Scott et al. [2004] showed that isolated CBS domains from ClC proteins are able to bind nucleotides. Similarly, Wellhauser et al. [2006] shows low affinity binding of ATP at the C terminus of ClC-5 that could be competed by $1 \mu \mathrm{M}$ AMP. Moreover, Bennettes et al. [2005] demonstrated that the activity of $\mathrm{ClC}-1$ is regulated by intracellular ATP and other nucleotides. A $5 \mathrm{mM}$ ATP concentration shifted open the gate by $\sim+50 \mathrm{mV}$ and this effect was enhanced by acidification of the intracellular solutions [Benettes et al., 2007; Tseng et al., 2007].

The recent structure of $\mathrm{ClC}-5$ in complex with ATP provides insight into the ligand binding site of CBS domains [Meyer et al., 2007]. As expected, ATP binds at the interface in the cleft opening of the two CBS domains forming several hydrogen bonds. The adenine base is stabilized, in a predominantly hydrophobic binding pocket, via a $\pi$-stacking interaction with Y617 on one face and by contacts with the aliphatic side chains of an isoleucine and a leucine residue on its other face (Figure 8) [Meyer et al., 2007]. The point mutation Y617A, which eliminates the stacking interaction with the adenine base, and the mutation D727A, which removes the side chain interactions with the two hydroxyl groups of the ribose moiety, both abolish ATP binding [Meyer et al., 2007]. In contrast, the mutation S618A, which removes the interaction of the side chain with the $\gamma$-phosphate, does not alter the binding affinity. Infact, the binding affinities, $K_{\mathrm{d}}$ 's $(\sim 100 \mu \mathrm{M})$, remain consistent among AMP, ADP, and ATP demonstrating the predominant contributions of the adenosine moiety and the ribose sugar in ClC-5 [Meyer et al., 2007]. 
Figure 8. Binding interactions formed between adenine ligands and the CBS domains of CLC-5 (A) and AMPK (B). Coloring: $\alpha$-helices - dark grey; $\beta$ - sheets - light grey; loops - white; carbon, nitrogen, oxygen and phosphorus atoms - cyan, blue, red, and purple, respectively; $\mathrm{H}$-bonds represented by dotted lines.

(A). CLC-5 in complex with ATP (PDB: 2J9L; Meyer et al., 2007). ATP stacking interaction of the adenine ring with Y617 of the CBS1 domain and hydrogen bonds between hydroxyl groups of the ribose moiety and D727 of the CBS2 domain. The corresponding residues are F439 (F443) and D501 (D538) in yCBS (hCBS).

Residue T596 of CLC-5 forms a pair of hydrogen bonds with the 6'-amino group and the 1'-aza group of the adenine ring and S618 and K726 interact with the $\alpha$ - and $\gamma$-phosphate groups, respectively, of the ATP ligand. The equivalent residues to S618 are D399 (D444) in yCBS (hCBS).

(B). Complex of AMPK with AMP (PDB ID: 2J9L; Day et al., 2007). Residue V225 of the CBS1 domain packs against the adenine ring, in place of the stacking interaction observed in the CLC-5-ATP complex (A), and hydrogen bonds are formed between S316 and the $\alpha$-phosphate and between D317 and hydroxyl groups of the ribose ring. The ribose sugar-binding motif in CBS2 stabilizes the hydroxyls of the ribose ring and the side chain of D317. The corresponding residues are F439 (F443) and D501 (D538) in yCBS (hCBS).

Residues A205 and A227 of AMPK form hydrogen bonds to the adenine ring of the AMP ligand and S226 interactions with the $\alpha$-phosphate. Residue S226 of AMPK corresponds to D399 (D444) in yCBS (hCBS). 
A

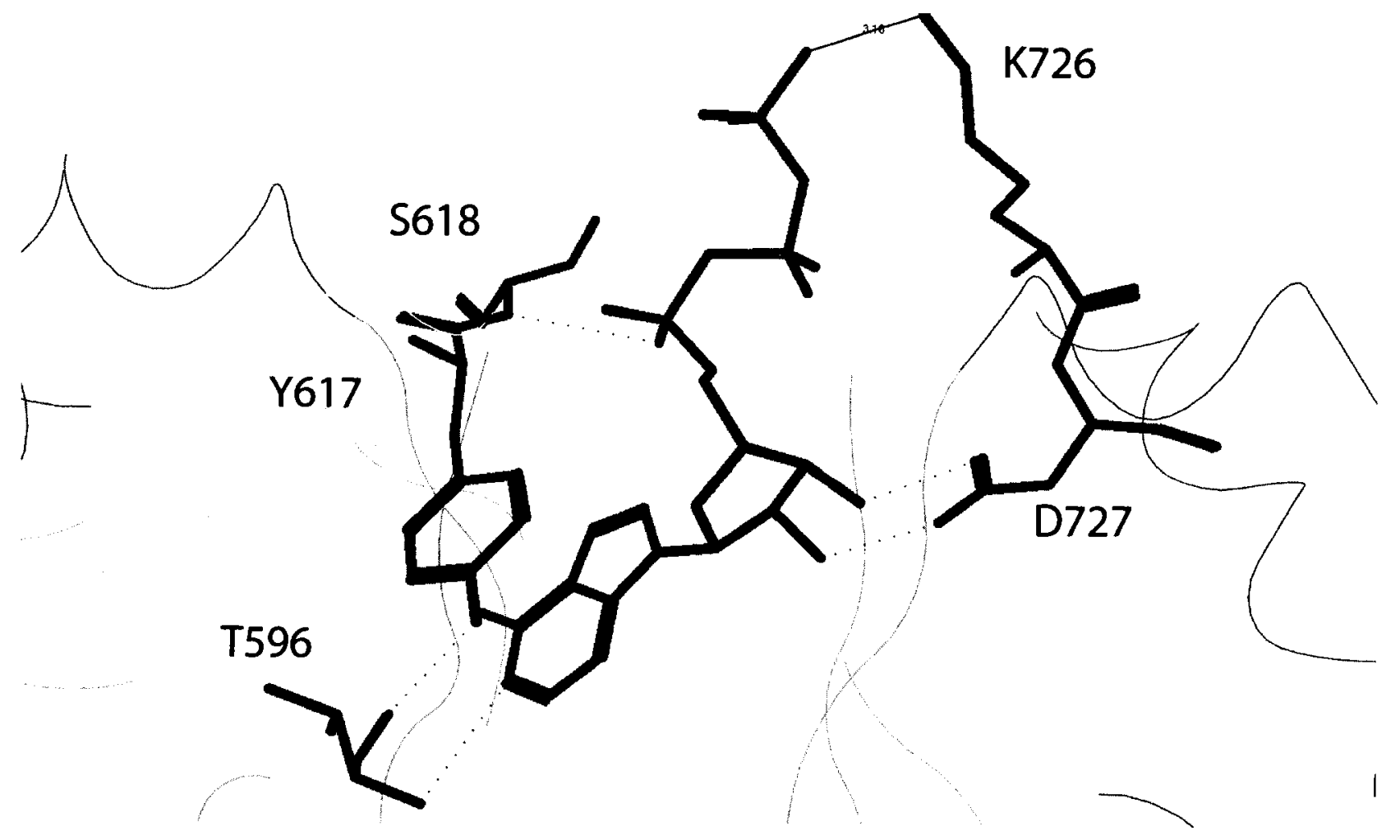

B

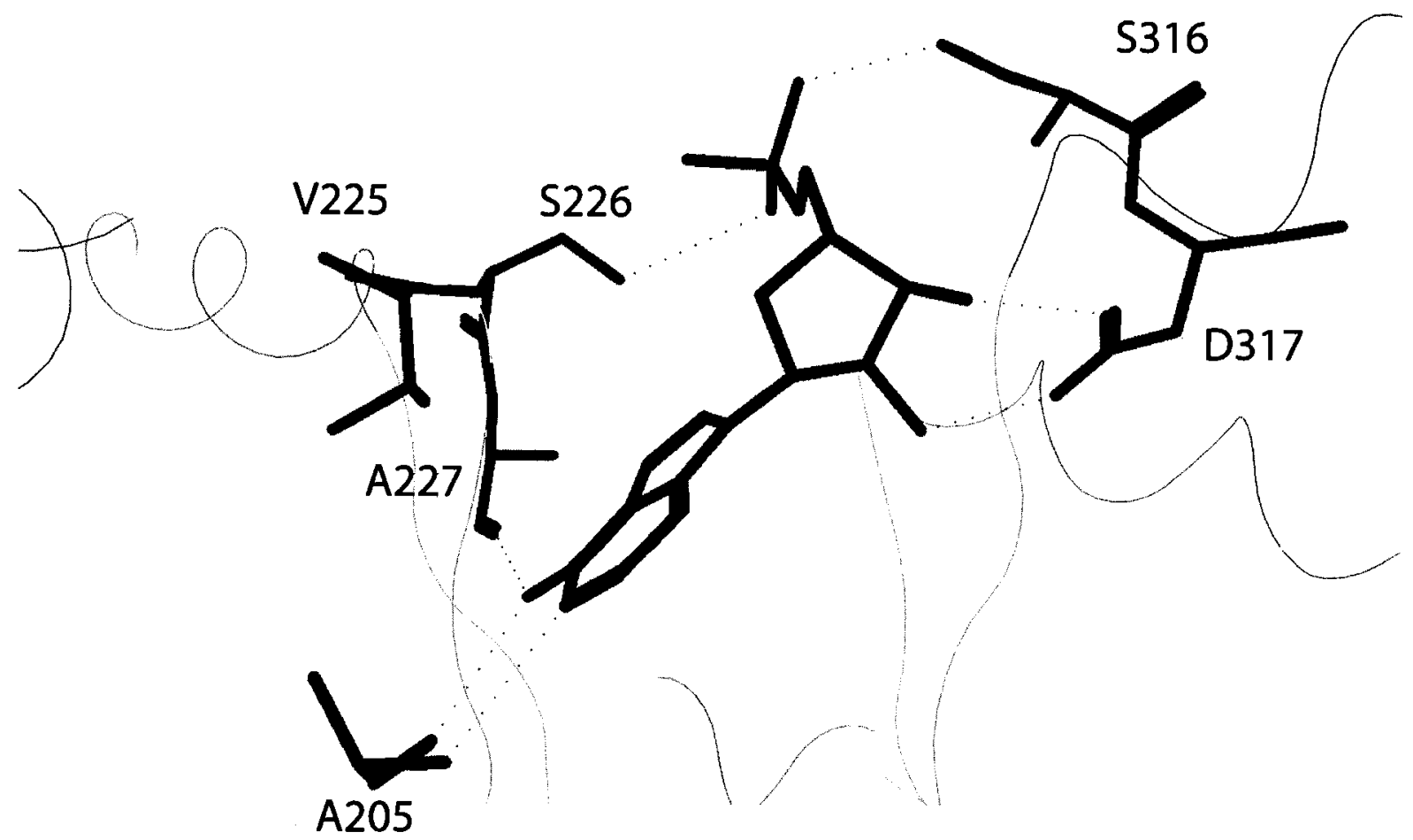


Residues Y617, S618 and D727 correspond to F439, D399, and D501, respectively, of yCBS and the corresponding F443, D444, and D538, of hCBS. The phenylalanine at position F439 of yCBS and F443 of hCBS offers the prospect of a conserved stacking interaction to the adenine ring in SAM. Coincidentally, though, D444 is a residue associated with the patient-derived D444N mutation of hCBS that results in a 15-fold decrease in SAM affinity.

\subsubsection{AMP-Activated Protein Kinase.}

The AMPK cascade functions as a sensor of cellular energy and plays an important role in restoring the cellular ATP balance following periods of metabolic stress [Kahn et al., 2005; Kemp et al., 2003]. Activation of AMPK, which occurs when the cellular energy balance drops (low ATP, high AMP concentrations), requires both binding of AMP and phosphorylation by AMPK kinase [Hardie et al., 2003]. Once activated, AMPK alters metabolic flux, via phosphorylation of regulatory metabolic enzymes, increasing the flux through catabolic pathways and decreasing that of anabolic pathways in an effort to raise the cellular ATP level [Hardie et al., 2003].

AMPK is a heterotrimeric protein, composed of alpha, beta and gamma subunits [Hardie et al., 1998]. The gamma subunit is comprised of four CBS domains, forming two pairs: CBS1 and -2 and CBS3 and -4 [Hardie et al., 1998]. The structure of a CBS3/4-domain pair from human AMPK-1 in complex with the physiological activator AMP was recently solved (Figure 8B) (Day et al., 2007). The nucleotide-binding site of AMPK is situated in the inter-domain cleft, as observed in the ClC-5 complex with AMP [Day et al., 2007; Metzler et al., 2007]. It is bounded by strands $\beta 1$ and $\beta 7$ of CBS4 and $\beta 2$ and $\beta 3$ of CBS3 and the side chains of residues of Thr200, Ile204, Val225 and Ile312 contact the AMP ligand [Day et al., 2007]. The adenine 
ring of moiety of ATP interacts with V225, the residue corresponding to Y617 of ClC-5. The hydroxyl groups of the ribose moiety are coordinated by the side chain of D317 (Yon et al., 2007). This also aligns with D727 of CLC-5, D501 in yCBS, and D538 in hCBS.

\subsection{Locating the allosteric-binding site in $h C B S$.}

It is apparent that the disease-associated point mutations in the CBS domains of diverse proteins likely exert their effect by reducing the binding of nucleotide-based allosteric activators, including, ATP, AMP and SAM, or altering the structure of the CBS domains, thereby resulting in regulatory disturbances. The structures of CBS domains from other proteins, particularly those in complex with the nucleotide ligands, offer valuable insight for the localization of the allosteric binding site of CBS and identification of residues involved in SAM binding. Analysis of multiple sequence alignments and the mapping of naturally-occurring mutations, that compromise ligand binding or allosteric activation, provide complementary clues. For example, the homocystinuriaassociated D444N mutation of hCBS (corresponding to D399 of yCBS), which reduces the binding affinity of the SAM allosteric activator, aligns with S226 of AMPK- $\gamma 1$, a residue that forms a direct hydrogen bond to the phosphate of AMP (Figure 10). Assuming that nucleotide molecules bind in the same pocket in CBS domains from diverse proteins, as observed in the ClC-5-ATP and AMPK-AMP complexes, hCBS residues corresponding to those observed to form H-bonding and hydrophobic interactions in ClC-5 and AMPK likely interact with SAM.

The AMPK-AMP structure revealed a ribose binding motif (GhxS/TxS/T, where $\mathrm{x}$ is any amino acid and $\mathrm{h}$ is a hydrophobic residue) that is conserved in the CBS4 domain of the $\gamma$ chains of AMPK from many species (Yon et al., 2007). Analysis of the full sequence of the $\gamma$ subunit of human AMPK demonstrated the presence of a similar sequence in the CBS1 domain 
(GMLTITD). The CBS domain pairs from human, rat, mouse, rabbit CBS and IMPDH all show these putative motifs in the second CBS domain (Figure 9) and the structure of the ClC-5-ATP complex confirms the interaction of the ribose hydroxyl moieties with residues D727 (D501 yCBS and D538 - hCBS) of this motif (GIITKKD). The two CBS domains of both hCBS and yCBS possess putative ribose-binding motifs, although that of CBS1 (GLVTLSE) demonstrates greater conservation than that of CBS2 (HIVTKMD) in the yeast enzyme, while that of CBS1 (GMVTLGN) demonstrates less conservation than that of CBS2 (GVVTAID) in the human enzyme. However, despite the conservation of this motif, the yeast enzyme, unlike hCBS, is reported not to be allosterically activated by SAM (Jhee et al., 2000b). The ability of SAM, or other adenine-containing ligands, to bind to yCBS has not been rigorously investigated and the reported lack of activation does not necessarily indicate that SAM does not bind to the regulatory domain of yCBS. The identification of a putative ribose-binding motif in CBS1 of yCBS offers the opportunity to investigate the role of these residues within the context of the model yeast enzyme.

Interestingly, the ribose-binding motif in CBS2 of hCBS is immediately adjacent to the peptide protected by SAM binding that was identified by peptide mass mapping following ${ }^{1} \mathrm{H} /{ }^{2} \mathrm{H}$-exchange (Figure 9) [Sen et al., 2005]. The nature of the other residues involved in binding, depends on the nature of the allosteric activator as, for example, positively-charged residue(s) may be required for binding of phosphate-bearing nucleotides, as observed both AMPK and IMPDH (R224P). Alternatively, negatively charged residue(s), may interact with positively-charged groups of the SAM ligand, as may be the role of D444 of hCBS (Figure 10). Due to the role of the regulatory domain of CBS in the oligomerization of the enzyme, it can be inferred that any change, whether within the CBS domains or residues interfacing with the 
domains may result in changes in the quaternary structure of CBS, in addition to its allosteric activation.

\subsection{Hypotheses and Objectives.}

The goal of my M.Sc. project was to investigate the mechanisms of regulation of CBS activity. The main objectives of this project were:

1. Development of an improved expression system for hCBS. This work was carried out in collaboration with Muluken Belew (M.Sc., 2008), who developed two of the five hCBS expression constructs described in section 9 of this thesis.

2. Characterization of the role of active-site residue S289 of yCBS in regulation of the chemistry of the versatile PLP cofactor. Construction of the S289A and S289D mutants and their $\mathrm{pH}$ titration were performed by Dr. Susan Aitken.

3. Investigation of the regulatory domain of yCBS and hCBS, with emphasis on the identification of residues involved in the allosteric activation of the enzyme. 
Figure 9. Sequence alignment of CBS domains. Putative ribose binding motifs are shown in bold. Both pairs of SNF4 (the yeast analogue of AMPK) CBS domains (CBS1+2 and CBS3+4) are aligned with mouse CBS, rabbit CBS, rat CBS, yCBS, and hCBS. Residues involved in interactions with AMP in AMPK-1 (CBS3+4) are underlined and bolded. Residue 444 of CBS, which is substituted by asparagine (D444N) in homocystinuria, is shown in underlined italics. The hCBS peptide (511-531) protected by SAM from ${ }^{1} \mathrm{H} /{ }^{2} \mathrm{H}$-exchange is shown in bold italics [Sen et al., 2005]. 
Human AMPK y1-1+2 Yeast SNF4-1+2

Human AMPK y $1-3+4$

Yeast SNF4-3+4

Mouse CBS

Rat CBS

Human CBS

Yeast CBS

Human IMPDHII

Human $\mathrm{ClC}-5$
Human AMPK y1-1+2 Yeast SNE4-1+2

Human AMPK y $1-3+4$

Yeast SNF4-3+4

Mouse CBS

Rat CBS

Human CBS

Yeast CBS

Human IMPDHII

Human $\mathrm{ClC}-5$

Human AMPK $\mathrm{Y}^{1-1+2}$

Yeast SNE4-1+2

Human AMPK y1-3+4

Yeast SNF4-3+4

Mouse CBS

Rat CBS

Human CBS

Yeast CBS

Human IMPDHII

Human $\mathrm{ClC}-5$

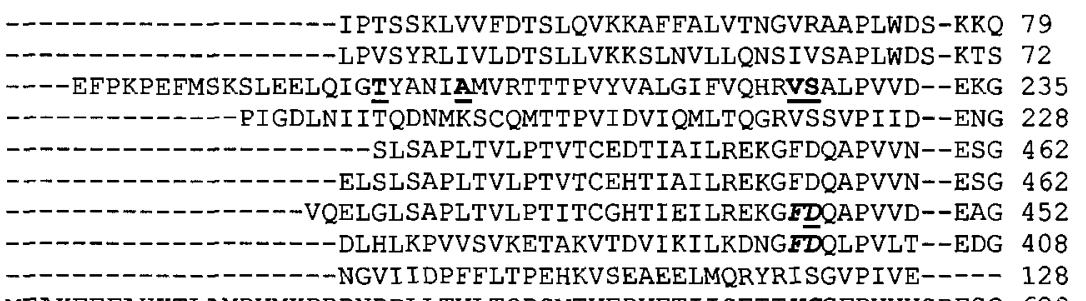

MEAKEEFAHKTLAMDVMKPRRNDPLLTVLTQDSMTVEDVETIISETTYSGFPVVVSRESQ 629

SEVGMLTITDFINILHRYYKS-------ALVQIYELEEHKIETWREVY-LQDSFKPLVCI 132 REAGILTTTDFINVIQYYFSNPD-----KFELVDKLQLDGLKDIERAL-GVDQLD-TASI 125 RVVDIYSKEDVIN-LAAEKTY--------NNLDVSVTKALQHRSHY-FEGVLK----C 279 YLINVYEAYDVLG-LIKGGIY--------NDLSLSVGEALMRRSDD-FEGVYT----C 274 AILGMVTLGNMLSSLLAGKVR--------PSDEVCKVLYKQFKPIH-LTDTLG----T 508 AILGMVTLGNMLSSLLAGKVR---------PSDEVCKVLYKQFKPIH-LTDTIG----M 508 VI LGMVTLGNMLSSLLAGKVQ--_- - PSDQVGKVIYKOFKOIR-LTDTLG----R 498 KLSGLVTLSELLRKLSINNSN---- ----NDNTIKGKYLDFKKLNNENDVSSYNENK 457 ----TLANRKLVGI ITNRDMR---------EISDYNAPISEHMTSEHLVTAAVG---- 169 RLVGFVLRRDLIISIENARKKQDGVVSTSIIYFTEHSPPLPPYTPPTLKLRNILDLSPFT 689

SPNASLFDAVSSLIRNKIHRLPVIDPESGN-----TLYILTHKRILKFLK---------- 177 HPSRPLFEACLKMLESRSGRI PLIDODEETHR-EIVVSVLTOYRILKFVA-...-_-_-_ 175 YLHETLETI INRLVEAEVHR--IVVVDEND----VVKGIVSLSDILQALV---------- 323 TKNDKLSTIMDNIRKARVHR--FFVVDDVG----RLVGVLTLSDILKYIL-_-_-_--_- 318 LSHILEMDHFALVVHEQIQYCSNGMSSKQQ----MVFGVVTAIDLLNFVAAREQTQT--- 561 LSHILEMDHFALVVHEQIQYRNNGVSSKQL----MVFGVVTAIDLLNFVAAREQTRK--- 561 LSHILEMDH FALV VHEQIQYHSTGKSSQRQ----MVFGVVTAIDLLNFVAAQERDQK--- 551 SGKKKFIKFDENSKLSDLNRFFEKNSSAVITDGLKPIHIVTKMDLLSYLA-- - - - - 507 ---TDLETAERILHEHRIEKLPLVDNSGRLS-----GLITIKDIEKVIE--- - - --- 210 VTDLTPMEIVVDI ERKLGLRQCLVTHNGRL------LGIITKKDVLKHIAQMANQDPDS I 743 
Figure 10. Structures of SAM and ATP. Atoms numbers, symbols, and stereochemistry are shown in SAM. 

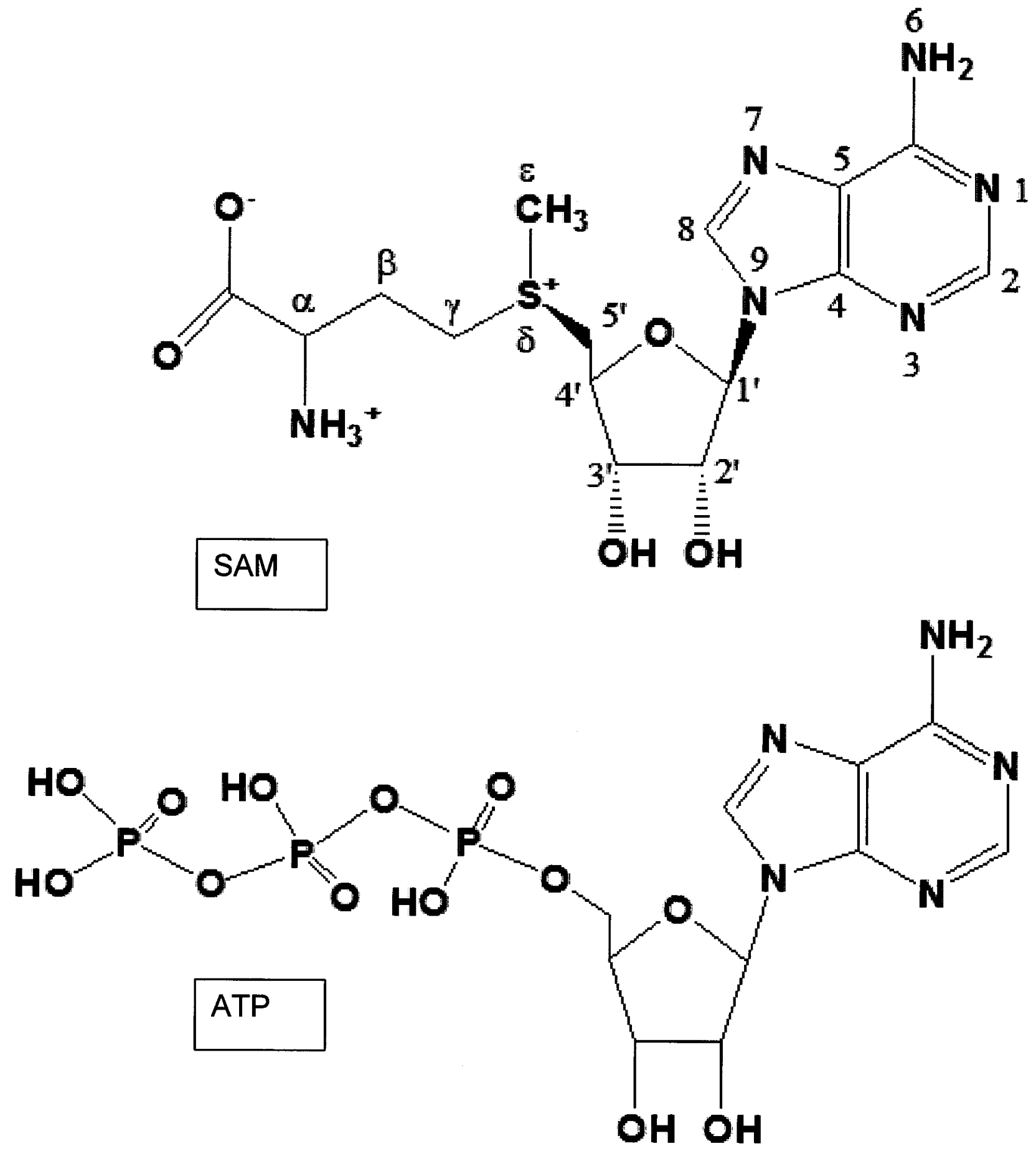
9. Kinetic Characterization of Recombinant Human Cystathionine $\beta$-Synthase Purified from $E$. coli. 


\subsection{Abstract.}

Cystathionine $\beta$-synthase (CBS) catalyzes the pyridoxal-5'-phosphate-dependent condensation of L-serine and L-homocysteine to form L-cystathionine in the first step of the transsulfuration pathway. Although effective expression systems for recombinant human CBS (hCBS) have been developed, they require multiple chromatographic steps as well as proteolytic cleavage to remove the fusion partner. Therefore, a series of five expression constructs, each incorporating a 6-His tag, were developed to enable the efficient purification of hCBS via immobilized-metal-ion affinity chromatography. Two of the constructs express hCBS in fusion with a protein partner, while the others bear only the affinity tag. The addition of an aminoterminal, 6-His tag, in the absence of a protein fusion partner and in the absence or presence of a protease-cleavable linker, was found to be sufficient for the purification of soluble hCBS and resulted in enzyme with $86-91 \%$ heme saturation and with activity similar to that reported for other hCBS expression constructs. The continuous assay for L-Cth production, employing cystathionine $\beta$-lyase and L-lactate dehydrogenase as coupling enzymes, was employed here for the first time to determine the steady-state kinetic parameters of hCBS, via global analysis, and revealed previously unreported substrate inhibition by L-Hcys $\left(K_{i}^{L-H c y s}=2.1 \pm 0.2 \mathrm{mM}\right)$. The kinetic parameters for the hCBS-catalyzed hydrolysis of L-Cth to L-Ser and L-Hcys were also determined and the $k_{c a t} / K_{m}{ }^{L-C t h}$ of this reaction is only $\sim 2$-fold lower than the $k_{c a t} / K_{m}{ }^{L-S e r}$ of the physiological, condensation reaction. 


\subsection{Introduction.}

Cystathionine $\beta$-synthase (CBS) catalyzes the condensation of L-serine (L-Ser) and Lhomocysteine (L-Hcys), a toxic metabolic intermediate of L-methionine (L-Met) metabolism, to produce L-cystathionine (L-Cth). Mammalian CBS is the only enzyme known to contain both pyridoxal 5'-phosphate (PLP), the catalytic cofactor, and heme [Kery et al., 1994], which is bound by the $\sim 70$-amino-acid, N-terminal domain [Meier et al., 2001; Taoka et al., 2002]. In contrast, CBS from yeast (Saccharomyces cervisiae) lacks the $\mathrm{N}$-terminal domain and does not bind heme [Jhee et al., 2000b; Maclean et al., 2000]. The gene encoding CBS is located on the $21^{\text {st }}$ chromosome in humans and is linked to the genetic disorders of homocystinuria and Down syndrome [Munke et al., 1988; Kraus et al., 1999; Kamoun, 2001]. Homocystinuria is an autosomal recessive disease, characterized by elevated plasma L-Hcys levels, with clinical manifestations including atherosclerosis-like vascular damage, mental retardation, osteoporosis, and skeletal abnormalities [Kraus et al., 1999; McCully, 2005].

As the substrate of the transmethylation and transsulfuration pathways, L-Hcys is situated at a metabolic branch point. The flux of L-Hcys through these competing pathways is regulated, via allosteric regulation, by the ubiquitous methyl donor $S$-adenosylmethionine (SAM) [Selhub and Miller, 1992; Finkelstein et al., 1975]. Human CBS is activated 2-3-fold by SAM, thereby increasing the flux of L-Hcys through the transsulfuration pathway when the cellular methionine pool exceeds the level required to maintain homeostasis [Finkelstein et al., 1975; Janosik et al., $2001 \mathrm{~b}$; Prudova et al., 2005]. The ultimate product of the transsulfuration pathway, L-Cys, is the immediate precursor of glutathione, the major compound responsible for maintaining cellular redox homeostasis. It has been proposed that the heme cofactor of hCBS plays a redoxregulatory role, thereby providing a potential feedback link between glutathione and the 
transsulfuration pathway [Banerjee and Zhou, 2005]. However, recent evidence suggests that a simple regulatory mechanism is unlikely and that heme-mediated regulation would be both temperature and pH-dependent [Pazicni et al., 2004; Pazicni et al., 2005; Cherney et al., 2007].

Purification of hCBS from mammalian tissues is complicated by its susceptibility to proteolysis and tendency to aggregate [Kraus et al., 1978; Kraus and Rosenberg, 1983]. Therefore, recombinant systems were developed for the expression of hCBS in Escherichia coli. For example, Bukovska et al. [1994] developed a system, in which hCBS is expressed as a fusion protein with $\beta$-galactosidase ( $\beta$-gal). Alternative $E$. coli expression systems, in which $\mathrm{hCBS}$ is expressed in conjunction with glutathione-S-transferase (GST), were subsequently developed by Shan and Kruger [Shan and Kruger, 1998] and by Janosik et al. [2001], although the hCBS produced retains an 11- or 23-residue extension at the amino-terminus, respectively, following proteolytic cleavage. Recently, a modified GST-hCBS expression construct was reported, which reduced the tag remaining at the amino-terminus of hCBS, following proteolytic cleavage, to a single glycine residue [Frank et al., 2008]. Expression of hCBS as a fusion protein with GST in $E$. coli has allowed yields of $2-11 \mathrm{mg} / \mathrm{L}$ and the variability in yield of purified hCBS reported is largely due to the aggregative tendency of the full-length enzyme [Janosik et al., 2001a; Frank et al., 2008; Taoka et al., 1998].

Each of the hCBS expression systems currently employed rely upon GST-based affinity purification and share a requirement for protease cleavage to remove the fusion partner, followed by an additional purification step. In contrast, the addition of a 6-His affinity tag to the carboxyterminus of truncated yeast CBS (ytCBS, residues 1-353) enabled its purification via a single chromatographic step and, as the kinetic parameters of this enzyme are identical to wild-type ytCBS, subsequent removal of the 6-His tag is not required [Aitken and Kirsch, 2004]. 
Therefore, to investigate the feasibility of employing Ni-nitrilo triacetic acid (Ni-NTA) affinity chromatography to purify hCBS, a 6-His tag was incorporated in a series of expression constructs: 6-His-GST/hCBS, 6-His-GFP/hCBS, 6-His-linker/hCBS, 6-His/hCBS and hCBS/6His. The constructs bearing an amino-terminal 6-His tag were found to be optimal for the rapid purification of active, soluble hCBS with heme saturation of $\sim 90 \%$. Steady-state kinetic parameters for the physiological condensation of L-Ser and L-Hcys were determined via global fit analysis, enabling quantification of substrate inhibition by L-Hcys. 
Figure 11. Schematic representation of the five hCBS expression constructs: (A) 6-HisGST/hCBS, (B) 6-His-GFP/hCBS, (C) 6-His-linker/hCBS, (D) 6-His/hCBS, and (E) hCBS/6His. 


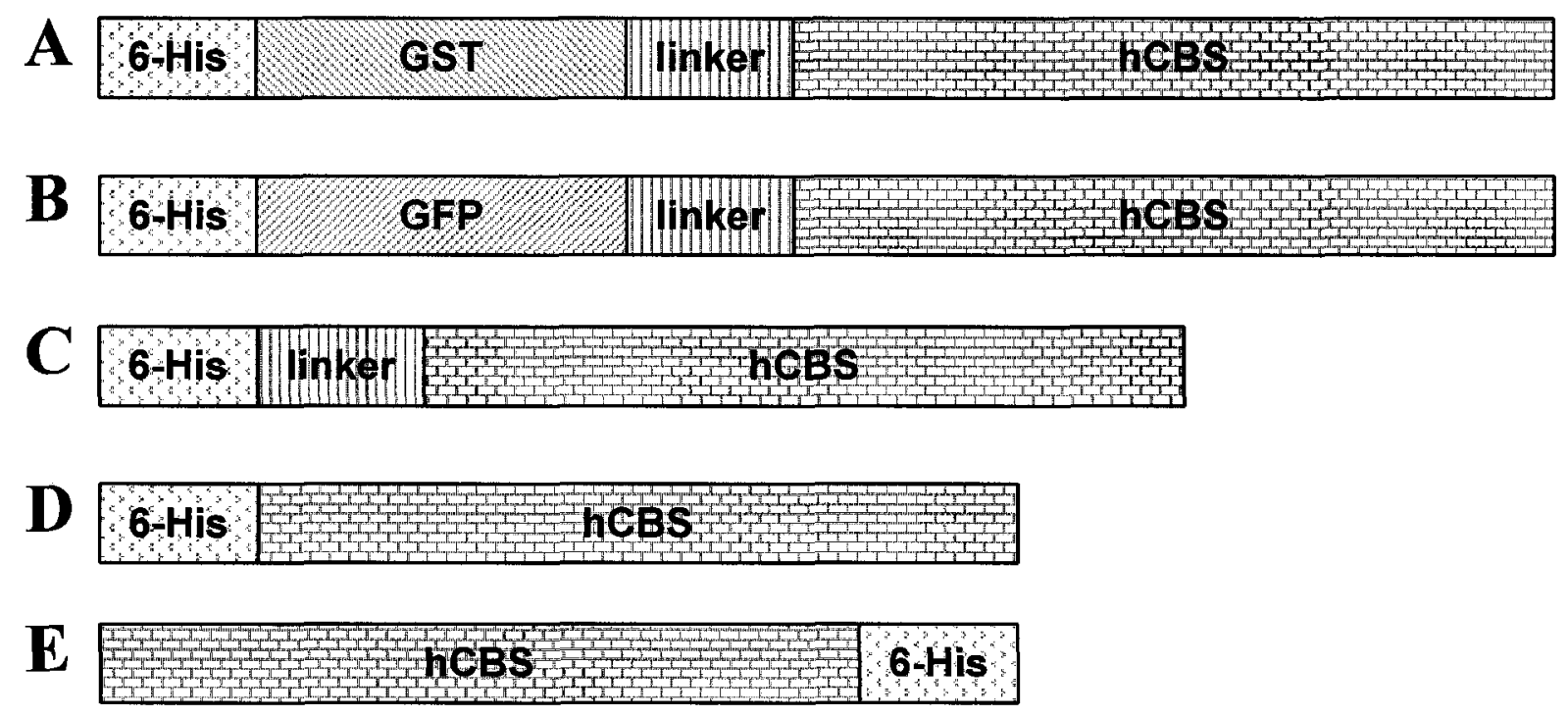




\subsection{Materials and Methods.}

\subsubsection{Reagents.}

$\delta$-Aminolevulinic acid ( $\delta$-ALA), L-lactate dehydrogenase (LDH), $\beta$-nicotinamide adenine dinucleotide ( $\beta$-NADH, reduced form), Tris- $\mathrm{HCl}$, thrombin, dithiothreitol (DTT), isopropyl $\beta$-D1-thiogalactopyranoside (IPTG), DNase I, lysozyme, L-Cth, L-Ser and L-Hcys thiolactone were purchased from Sigma-Aldrich. L-Hcys was prepared from the thiolactone via the method described by Kashiwamata and Greenberg [1970]. Protease inhibitor tablets were a Roche product and nickel-nitrilotriacetic acid (Ni-NTA) resin was from Qiagen. Potassium phosphate (monobasic and dibasic), 5, 5'-dithio-bis-(2-nitrobenzoic acid) (DTNB) and imidazole were Fisher Scientific products. The 6-His-tagged cystathionine $\beta$-lyase (CBL) coupling enzyme, employed in the CBS assay, was expressed and purified as described previously [Aitken and Krisch, 2004]. Oligonucleotide primers were synthesized by Integrated DNA Technologies Inc. All restriction endonucleases and T4 DNA ligase were obtained from New England BioLabs. The pGEX4T-hCBS expression construct was the generous gift of Dr. Warren Kruger (Fox Chase Cancer Center, Philadelphia).

\subsubsection{Preparation of the 6-His-GST/hCBS Expression Construct.}

The 6-His-GST/hCBS expression constructed was produced by Dr. Susan Aitken. The sequence spanning both the GST and hCBS coding regions was amplified from the pGEX4ThCBS vector with the GSTf-His-NcoI (CAT GCC ATG GCC CAC CAC CAT CAC CAC CAT TCC CCT ATA CTA GGT TAT TGG) and hfCBSr-Xbal primers (GCT CTA GAT CAC TTC TGG TCC CGC TCC TGG GCG GCC ACG), which comprise a NcoI restriction endonuclease 
site and 6-His tag and a $X b a I$ site, respectively, and introduced at the corresponding sites of the pTrc-99a expression vector, under control of the IPTG-inducible trc promoter, to produce the 6His-GST/hCBS expression construct [Shan and Kruger, 1998].

\subsubsection{Preparation of the 6-His/hCBS and 6-His-GFP/hCBS Expression Constructs.}

The 6-His/hCBS and 6-His-GFP/hCBS expression constructed were created by Muluken Belew. The $e G F P-1$ gene was amplified from the pEGFP-1 plasmid (Clontech) using the GFPFc-NcoI-His (5'-TAG ACC ATG GCG CAT CAT CAC CAT CAC CAT ATG GTG AGC AAG GGC GAG-3') and GFP-Rc-KpnI (5'-GGC ATG GAC GAG CTG TAC AAG TAA-3') primers and inserted between the $\mathrm{NcoI}$ and $\mathrm{KpnI}$ sites of pTrc-99a. A 6-His tag was incorporated immediately following the start codon of GFP, in the GFP-Fc-NcoI-His primer. The pTrc99aGFP construct was subsequently modified to introduce a linker sequence, encoding a thrombin proteolytic cleavage site, at the KpnI site at the 3' end of the eGFP-1 gene. The linker was designed to incorporate a unique SpeI restriction site, not present in the vector, such that digestion with SpeI endonuclease, followed by re-ligation of the vector, would enable selection of a construct containing a single copy of the linker. The KpnI site, at the 3' end of the linker, was subsequently converted to a NdeI site via whole-plasmid, DpnI-mediated mutagenesis [Weiner et al., 1994; Kery et al., 1995]. The pTrc99a vector contains a NdeI site, outside of the multiple cloning site, which was subsequently converted to a BgllI site. The hCBS gene was amplified from the pGEX4T-hCBS vector with the hCBS-Fc-NdeI (5'-GAC AAA CAT ATG CCT TCT GAG ACC CCC C-3') and hCBS-Rc-SalI (5'-GGA TCC GAT CGA CTT CAC TTC TGG TCC CGC-3') primers. The pTrc99a/GFP-linker vector contains a pair of NdeI sites, one situated at the junction of the 6-His tag and the GFP gene and the second at the 3' end of linker 
sequence. Therefore, insertion of the amplified hCBS sequence between the $N d e \mathrm{I}$ and $S a l \mathrm{I}$ sites of this plasmid substituted the GFP-linker sequence with the hCBS amplicon to produce the 6His/hCBS expression construct. The $N d e I$ site between 6-His tag and GFP of the pTrc99a/GFPlinker plasmid was subsequently changed to a $B m t I$ site via whole-plasmid, DpnI-mediated mutagenesis and insertion of the amplified hCBS sequence at the $N d e I$ and $S a l I$ sites of this plasmid produced the 6-His-GFP/hCBS expression construct.

\subsubsection{Preparation of the hCBS/6-His and 6-His-linker/hCBS Expression Constructs.}

Whole-plasmid, DpnI-mediated mutagenesis was employed to replace the NcoI site of the pTrc-99a multiple cloning site with a $N d e I$ site and to remove the existing $N d e I$ site to produce a modified pTrc-99a vector. The hCBS sequence was amplified from the pGEX4T-hCBS vector with the hCBS-Fc-NdeI (GGG AAT TCC ATA TGC CTT CT GAGA CCC CC) and hCBS-RcHis-SalI (TAT ATG TCG ACA TGA TGA TGA TGA TGA TGT CAC TTC TGG TCC CGC TCC TGG GCG GC) primers, the latter encoding a 6-His tag, and inserted at the NdeI and SalI sites of the modified pTrc-99a vector to produce the hCBS/6-His expression construct. The modified pTrc-99a expression vector was also altered to incorporate a sequence encoding a 6-His tag and a Factor Xa protease site at the 5' end of the poly-linker. A mixture of $1 \mathrm{nmol}$ of each of the linker-top (5'-TAG CCA TCA TCA TCA TCA TCA TAG CAG CGG CCA TAT CGA AGG TCG TCA) and linker-bottom (5'-TAT GAC GAC CTT CGA TAT GGC CGC TGC TAT GAT GAT GAT GAT GAT GGC) oligonucleotide primers were incubated at $70^{\circ} \mathrm{C}$ for $5 \mathrm{~min}$, annealing buffer was added (to a final concentration of $100 \mathrm{mM}$ Tris $\mathrm{HCl}, \mathrm{pH} \mathrm{7.5,} 70 \mathrm{mM}$ $\mathrm{MgCl}_{2}$ ) and the mixture was incubated at $70^{\circ} \mathrm{C}$ for 5 min prior to gradual cooling to $25^{\circ} \mathrm{C}$, over a period of one hour. The annealed linker was inserted at the introduced NdeI site of the modified 
pTrc-99a plasmid, the translation initiation site was subsequently reintroduced via wholeplasmid, DpnI-mediated mutagenesis and the hCBS sequence, amplified from the hCBS/6-His construct with the pSECseq0 (GGC GTC AGG CAG CCA TCG GAA GCT G) and hCBS-RcSalI vector and gene-specific primers, respectively, was inserted at the $N d e I$ and SalI sites to produce the 6-His-linker/hCBS expression construct.

\subsubsection{Protein Expression.}

All hCBS constructs were expressed and purified using the same procedure. A $100-\mathrm{mL}$, overnight culture of $E$. coli strain DH10b (Amersham), containing the hCBS expression construct, was grown in LB media containing $100 \mu \mathrm{g} / \mathrm{mL}$ ampicillin. Three liters of LB media, containing $75 \mathrm{mg} / \mathrm{L} \delta$-aminolevulinic acid ( $\delta$-ALA), were inoculated with the overnight culture media (at a 1:50 inoculant-to-media ratio) in baffled, 2.8-L Fernbach flasks (1 L per flask) [29]. Cells were grown at $30^{\circ} \mathrm{C}$ and IPTG was added to a final concentration of $0.2 \mathrm{mM}$ when the $\mathrm{OD}_{600}$ reached 0.5 . The cells were grown at $30^{\circ} \mathrm{C}$ for a further $16 \mathrm{~h}$ and harvested by centrifugation at $4000 \mathrm{rpm}$ for $10 \mathrm{~min}$ at $4^{\circ} \mathrm{C}$. The cell pellets were washed by resuspension in $100 \mathrm{~mL}$ of $0.85 \% \mathrm{NaCl}$ followed by centrifugation at $4000 \mathrm{rpm}$ for $10 \mathrm{~min}$. Cell pellets were resuspended in $40 \mathrm{~mL}$ of lysis buffer $(50 \mathrm{mM}$ potassium phosphate, $\mathrm{pH} 7.8,10 \mathrm{mM}$ imidazole, $20 \mu \mathrm{M}$ PLP), containing one "EDTA-free" protease inhibitor tablet (Roche), $1 \mathrm{mg} / \mathrm{mL}$ lysozyme and $10 \mu \mathrm{g} / \mathrm{mL}$ DNase I, and incubated at room temperature for 20 minutes prior to sonication ( 8 cycles of $30 \mathrm{sec}$ at $50 \%$ duty cycle; Vibra Cell sonicator, Sonic and Material, Inc.). The cell lysate was centrifuged at $15000 \mathrm{rpm}$ for 45 minutes at $4^{\circ} \mathrm{C}$ and the supernatant was loaded on a 2-mL Ni-nitrilo triacetic acid (Ni-NTA) column, equilibrated with lysis buffer. The column was subsequently washed with at least 40 column volumes of lysis buffer and the protein was eluted 
with a 200-mL linear gradient of 10-200 mM imidazole in lysis buffer. Fractions containing pure hCBS protein were pooled, concentrated and dialyzed against hCBS storage buffer (50 mM potassium phosphate buffer, $20 \mu \mathrm{M}$ PLP, and $1 \mathrm{mM}$ EDTA), glycerol was added to $20 \%$ (v/v) and the aliquoted enzyme was stored at $-80^{\circ} \mathrm{C}$. The protein concentration was determined by the Bradford method (BioRad), using bovine serum albumin (BSA) as a standard [Bradford, 1970]. The heme content of each enzyme was quantified using the pyridine hemochromogen method using hemin hydrochloride as a standard [Frank et al., 2008; Morrison and Horie, 1965]. The purified 6-His-GST/hCBS and 6-His-GFP/hCBS proteins were treated with thrombin to remove the fusion partner. The protein was incubated for $6 \mathrm{~h}$ with 1 unit of thrombin (Sigma-Aldrich) per mg of hCBS fusion protein in hCBS storage buffer and the cleavage products were separated via Ni-NTA chromatography prior to measurements of activity and heme saturation. Similarly, the 6-His-linker/hCBS enzyme was digested with $20 \mu \mathrm{g}$ of Factor Xa protease (New England BioLabs) per $\mathrm{mg}$ of hCBS protein in protease buffer $(20 \mathrm{mM}$ Tris- $\mathrm{HCl}$, $\mathrm{pH} 8.0,100 \mathrm{mM} \mathrm{NaCl}, 2$ $\mathrm{mM} \mathrm{CaCl}_{2}$ ) for $6 \mathrm{~h}$ to remove the amino-terminal 6-His tag and linker sequence.

\subsubsection{Enzyme Assays.}

Enzyme activity was measured in a total volume of $100 \mu \mathrm{L}$ at $25^{\circ} \mathrm{C}$ with a Molecular Devices Spectramax 390 spectrophotometer. The assay buffer comprised $50 \mathrm{mM}$ Tris, $\mathrm{pH}$ 8.6, and $20 \mu \mathrm{M}$ PLP. A background rate, for all components except the hCBS enzyme, was recorded for each sample before initiating the reaction by the addition of hCBS. Data was fit by nonlinear regression with SAS (SAS Institute, Cary, NC). The reverse-physiological hydrolysis of L-Cth to L-Ser and L-Hcys by hCBS was detected via the reaction of 5, 5'-dithiobis-(2-nitrobenzoic acid) (DTNB) with the free thiol of the L-Hcys product [Aitken and Kirsch, 2003]. Reactions were 
carried out in assay buffer containing $2 \mathrm{mM}$ DTNB and $0.01-6.3 \mathrm{mM} \mathrm{L}-\mathrm{Cth}$ and $0.44-0.53 \mu \mathrm{M}$ hCBS. Absorbance changes were monitored at $412 \mathrm{~nm}\left(\Delta \varepsilon_{412}=13,600 \mathrm{M}^{-1} \mathrm{~cm}^{-1}\right)$. Values of $k_{\text {cat }}$ and $K_{m}$ were obtained by fitting the data to the Michaelis-Menten equation, and $k_{c a t} / K_{m}$ was obtained independently from equation 1 .

$$
\frac{v}{[E]}=\frac{k_{c a t} / K_{m} \times[S]}{1+\left[S / / K_{m}\right.}
$$

The CBS-catalyzed condensation of L-Ser and L-Hcys to produce L-Cth was assayed via the continuous CBL-LDH assay [Aitken and Kirsch, 2003]. Reactions were carried out in assay buffer containing 1.5 mM NADH, 1.6 $\mu \mathrm{M} \mathrm{CBL}, 1.4 \mu \mathrm{M} \mathrm{LDH}, 0.1-11.3 \mathrm{mM} \mathrm{L-Hcys}$ and 0.2-30 $\mathrm{mM} \mathrm{L-Ser}$ and were initiated by the addition of $0.88-1.06 \mu \mathrm{M} \mathrm{hCBS}$. Kinetic parameters were determined, via global analysis, from the fit of the data to equation 2, in which $K_{i}^{L-H c y s}$ is the inhibition constant for substrate inhibition by L-Hcys [Jhee et al., 2000b].

$$
\frac{v}{[E]}=\frac{k_{c a t}[L-S e r][L-H c y s]}{K_{m}^{L-H c y s}[L-S e r]+K_{m}^{L-S e r}\left[L-H c y s\left(1+\frac{[L-H c y s}{K_{i}^{L-H c y s}}\right)+[L-S e r][L-H c y s]\right.}
$$




\subsection{Results.}

\subsubsection{Evaluation of hCBS expression constructs.}

Five constructs were developed and compared in an attempt to create an hCBS expression system enabling Ni-NTA affinity chromatography to be employed for purification and to investigate the need for co-expression with a fusion partner (Figure 11). The constructs all include a 6-His tag, but differ in its location and the presence of a fusion partner and linker. The 6-His-GST/hCBS and 6-His-GFP/hCBS constructs bear the affinity tag at the amino-terminus of the GST or GFP proteins, respectively, which are expressed in fusion with hCBS. In contrast, 6His/hCBS and hCBS/6-His include only a non-cleavable, amino- or carboxy-terminal 6-His tag, respectively. The 6-His-linker/hCBS construct, which also encodes an amino-terminal 6-His tag, is distinguished from the 6-His/hCBS construct by the presence of a 9-amino-acid linker, containing a Factor Xa cleavage site, between the affinity tag and the hCBS sequence (Figure 11).

Purification of 6-His-GST/hCBS and 6-His-GFP/hCBS, via Ni-NTA chromatography, resulted in 60 and 40-fold increases in specific activity, respectively (Table 1), compared to the crude lysate, and yielded the predicted fusion protein of $\sim 90 \mathrm{kDa}$, as estimated by SDS-PAGE (Figure 12). Thrombin treatment of both proteins resulted in loss of the $\sim 90-\mathrm{kDa}$ protein band with concomitant production of the expected 26 or 28 and $63 \mathrm{kDa}$ bands, corresponding to GST, GFP and hCBS, respectively (Figure 12). The 6-His/hCBS and 6-His-linker/hCBS proteins have molecular weights of $\sim 63-\mathrm{kDa}$ and purification with Ni-NTA resin resulted in 70 and 50 -fold increases in specific activity, respectively (Table 1), compared to the crude lyase, and produced protein that is $>90 \%$ pure, as demonstrated by SDS-PAGE (Figure 12). Treatment of the 6-Hislinker/hCBS protein with Factor Xa, protease effectively removes the 1.8 -kDa amino-terminal 
sequence (MHHHHHHSSGHIEGR) to produce hCBS enzyme bearing only a single histidine residue extension at the amino terminus (Figure 12). Expression of the hCBS/6-His construct, with the carboxy-terminal, 6-His tag, did not produce soluble protein, suggesting that the presence of the affinity tag in this location destabilized the oligomeric structure of the enzyme. The yield of hCBS, for each of the four soluble constructs, was in the $0.5-2.2 \mathrm{mg} / \mathrm{L}$ range and the heme saturation was between 76-96\% (Table 1).

The four soluble enzymes were assayed using the continuous CBS assay, following thrombin cleavage to remove the GST and GFP fusion partners of 6-His-GST/hCBS and 6-HisGFP/hCBS, respectively, and before and after removal of the affinity tag and linker from 6-Hislinker/hCBS [Aitken and Kirsch, 2003]. Comparison of their kinetic parameters at $25^{\circ} \mathrm{C}$ demonstrates that the $k_{c a t} / K_{m}{ }^{L-S e r}$ and $k_{c a t} K_{m}{ }^{L-H c y s}$ are between $150-250$ and $1200-3500 \mathrm{M}^{-1} \mathrm{~s}^{-1}$, respectively (Table 2). Although thrombin cleavage of the GST and GFP fusion proteins results in a residual, amino-terminal tag of 11 (GSPEFPGRPAG) and 3 (GSH) amino acids, respectively, the $k_{c a t} / K_{m}{ }^{L-S e r}$ and $k_{c a t} / K_{m}{ }^{L-H c y s}$ of these enzymes differ only marginally. The $k_{c a t} / K_{m}{ }^{L-S e r}$ of the affinity tagged enzymes, 6-His/hCBS and 6-His-linker/hCBS, was similar to that of hCBS from the cleaved GST and GFP fusion proteins, while the $k_{\text {car }} K_{m}{ }^{L-H c y s}$ of the tagged enzymes was 2-3-fold greater. Removal of the 6-His tag and linker from 6-His-linker/hCBS did not alter the kinetic parameters of the enzyme, demonstrating that proteolytic cleavage is not required for kinetic characterization (Table 2). Therefore, the two tagged enzymes, which require only a single chromatographic step to produce homogeneous enzyme, were selected for further characterization. 


\subsubsection{Steady-state characterization of hCBS.}

The presence of substrate inhibition by L-Hcys is evident in Figure 13. Therefore, global analysis, in which both substrates are varied simultaneously, was employed and the data was fit to equation 2 , which incorporates a substrate inhibition term $\left(K_{i}^{L-H c y s}\right)$, as described by Jhee et al. [2000b] for the model yCBS enzyme. With the exception of a 1.6-fold difference in $K_{m}{ }^{L-H c y s}$, the kinetic parameters of the $\beta$-replacement reaction catalyzed by 6-His/hCBS and 6His-linker/hCBS are identical, within their experimental error (Table 3), demonstrating that the addition of the 9-residue linker between the 6-His tag and hCBS does not alter activity. The ability of 6-His/hCBS and 6-His-linker/hCBS to catalyze the hydrolysis of L-Cth, producing LSer and L-Hcys, was also measured and the $k_{c a t}$ and $K_{m}{ }^{L-C t h}$ of the hydrolysis reaction are $\sim 70-$ fold and $\sim 40$-fold lower than the $k_{c a t}$ and $K_{m}{ }^{L-S e r}$, respectively, of the condensation reaction (Table 3). 


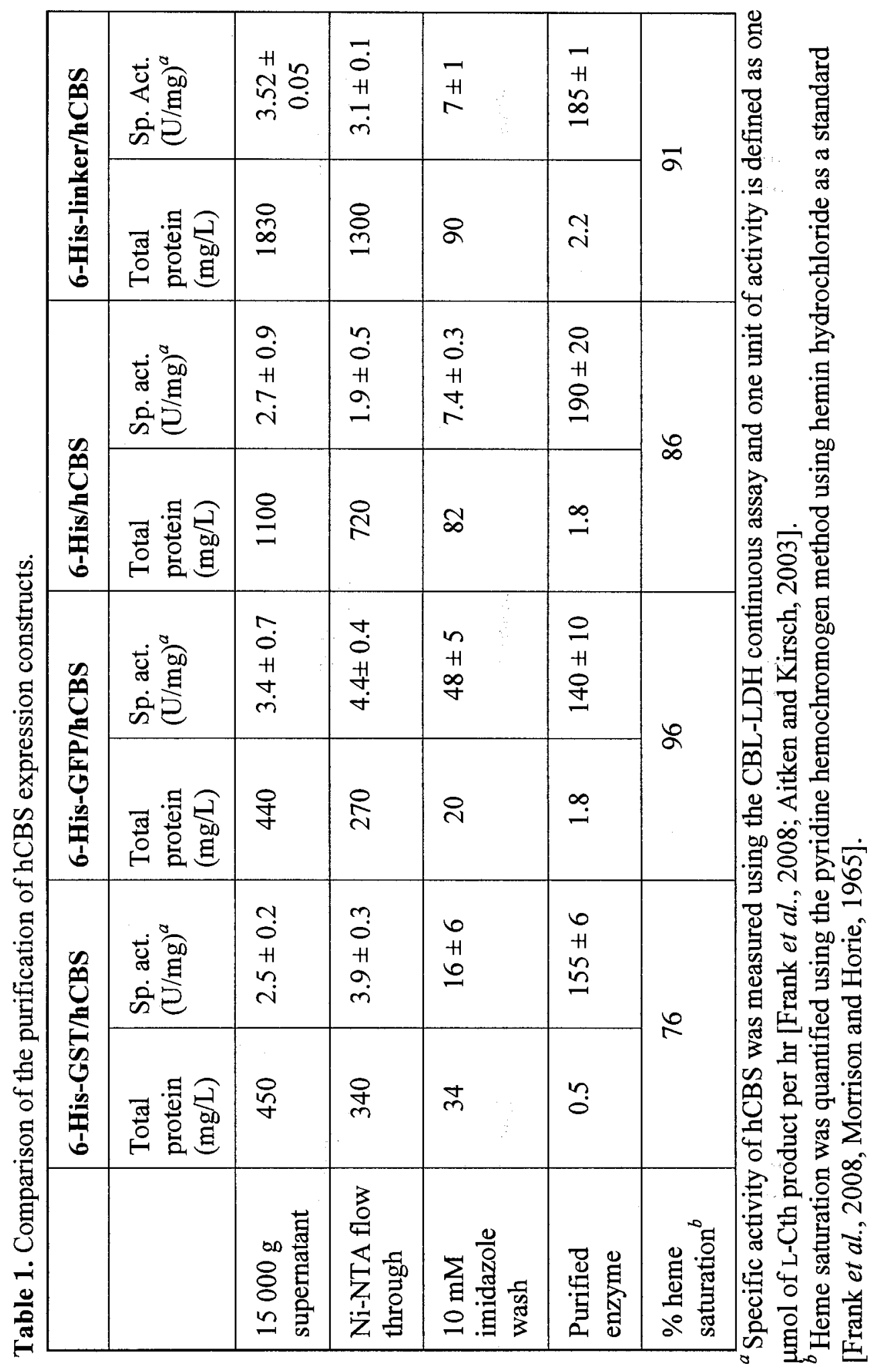




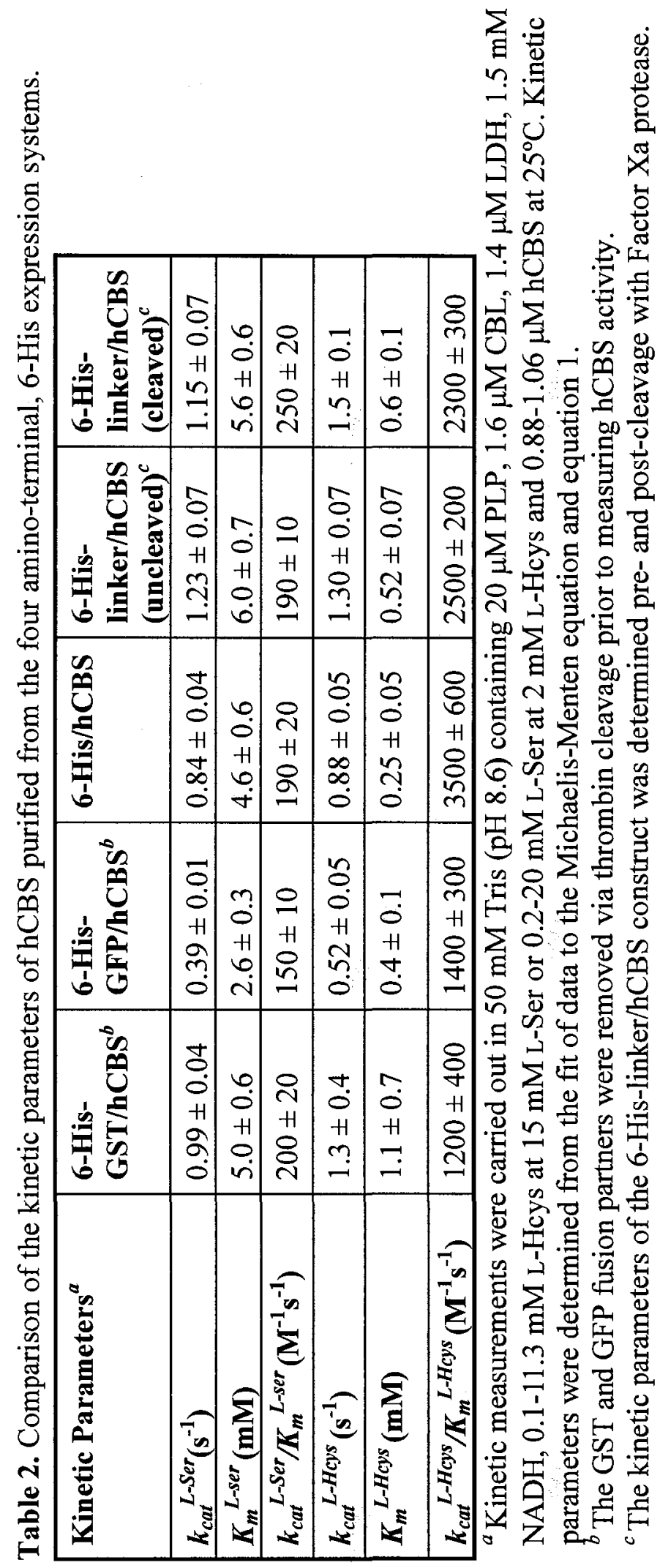


Table 3. Steady-state kinetic parameters of 6-His/hCBS and 6-His-linker/hCBS.

\begin{tabular}{|c|c|c|}
\hline Kinetic Parameter & 6-His/hCBS & 6-His-linker/hCBS \\
\hline \multicolumn{3}{|c|}{ L-Ser + L-Hcys $\rightarrow$ L-Cth ${ }^{a}$} \\
\hline$k_{c a t}\left(\mathrm{~s}^{-1}\right)$ & $1.67 \pm 0.07$ & $1.69 \pm 0.06$ \\
\hline$K_{m}{ }^{L-S e r}(\mathrm{mM})$ & $5.0 \pm 0.5$ & $5.1 \pm 0.5$ \\
\hline$K_{m}{ }^{L-H c y s}(\mathrm{mM})$ & $0.43 \pm 0.04$ & $0.27 \pm 0.03$ \\
\hline$k_{c a t} / K_{m}{ }^{L-S e r}\left(M^{-1} \mathrm{~s}^{-1}\right)$ & $340 \pm 30$ & $330 \pm 30$ \\
\hline$k_{c a t} / K_{m}{ }^{L-H c y s}\left(\mathrm{M}^{-1} \mathrm{~s}^{-1}\right)$ & $3800 \pm 200$ & $6400 \pm 400$ \\
\hline$K_{i}^{L-H c y s}(\mathrm{mM})$ & $2.1 \pm 0.2$ & $2.0 \pm 0.3$ \\
\hline \multicolumn{3}{|c|}{ L-Cth $\rightarrow$ L-Ser+ L-Hcys ${ }^{b}$} \\
\hline$k_{c a t}\left(\mathbf{s}^{-1}\right)$ & $0.0240 \pm 0.0006$ & $0.0291 \pm 0.0008$ \\
\hline$K_{m}{ }^{L-C C h}(\mathbf{m M})$ & $0.13 \pm 0.02$ & $0.19 \pm 0.02$ \\
\hline$k_{c a t} / K_{m}^{L-C t h}\left(M^{-1} s^{-1}\right)$ & $180 \pm 20$ & $160 \pm 20$ \\
\hline
\end{tabular}

${ }^{a}$ Kinetic measurements were carried out in $50 \mathrm{mM}$ Tris (pH 8.6) containing $20 \mu \mathrm{M}$ PLP, 1.5 mM NADH, 1.6 $\mu \mathrm{M}$ CBL, 1.4 $\mu \mathrm{M}$ LDH, 0.1-11.3 mM L-Hcys, 0.2-30 mM L-Ser and $0.88-1.06 \mu \mathrm{M} h \mathrm{CBS}$ at $25^{\circ} \mathrm{C}$. Data were fit to equation 2 .

${ }^{b}$ Kinetic measurements were carried out in $50 \mathrm{mM}$ Tris ( $\mathrm{pH} \mathrm{8.6)} \mathrm{containing} 20 \mu \mathrm{M}$ PLP, $2 \mathrm{mM}$ DTNB, 0.01-6.3 mM L-Cth, and 0.44-0.53 $\mu \mathrm{M}$ hCBS. Data were fit to the Michaelis-Menten equation and equation 1. 
Figure 12. SDS-PAGE analysis of purified recombinant hCBS. Lanes: 1 and $\mathbf{9}$, protein molecular weight ladder (Benchmark, Invitrogen); 2, 6-His-GST/hCBS; 3, thrombintreated 6-His-GST/hCBS; 4, 6-His-GFP/hCBS $(5 \mu \mathrm{g})$; 5, thrombin-treated 6-HisGFP/hCBS; 6, 6-His/hCBS; 7, 6-His-linker/hCBS; 8, 6-His-linker/hCBS treated with Factor $\mathrm{Xa}$. The $10 \%$ polyacrylamide gel was loaded with $5 \mu \mathrm{g}$ protein per lane and stained with Coomassie brilliant blue. 


\section{$\begin{array}{lllllllll}1 & 2 & 3 & 4 & 5 & 6 & 7 & 8 & 9\end{array}$}

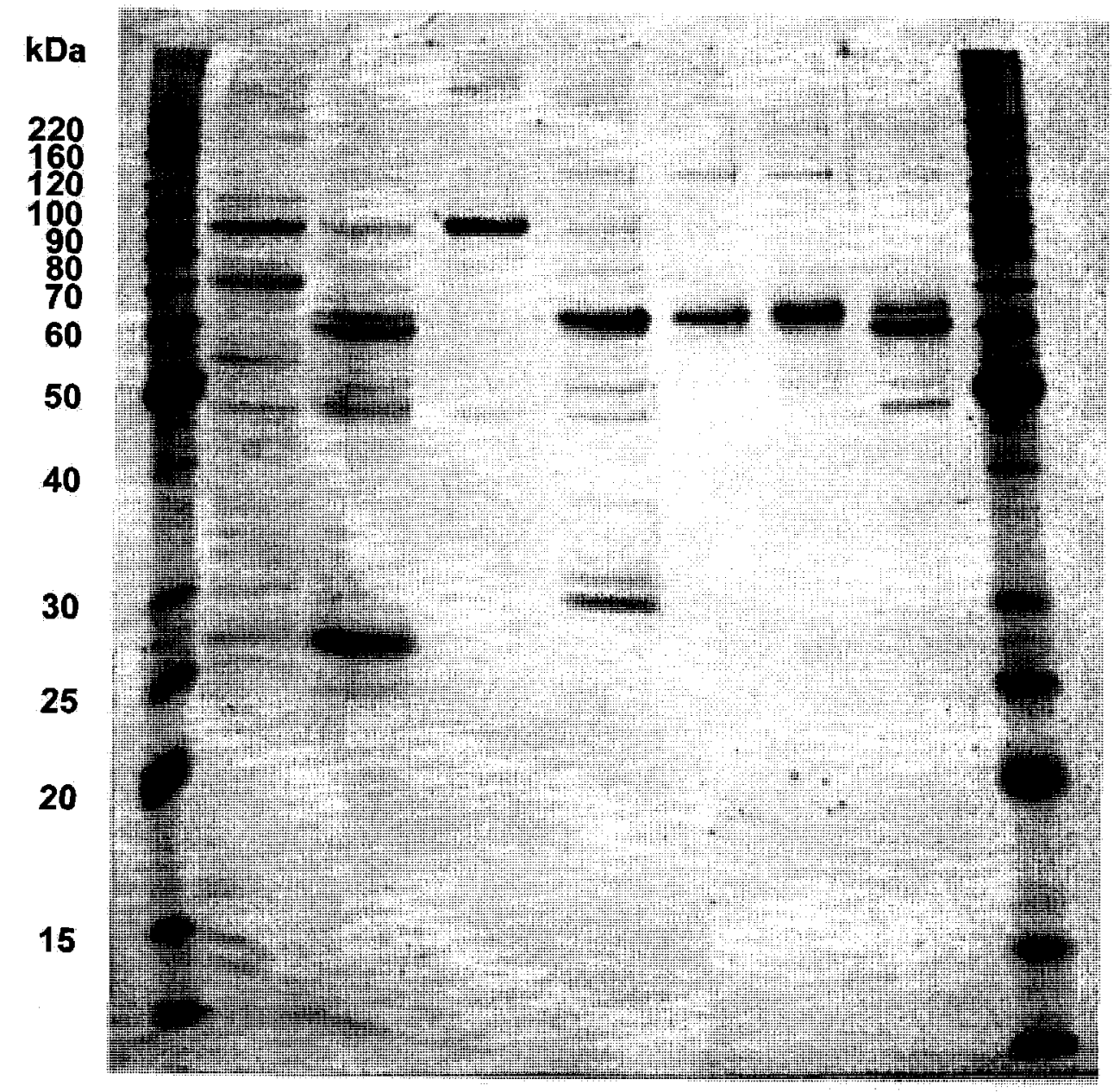


Figure 13. Steady-state initial velocity kinetics for hCBS showing the dependence of the reaction on [L-Hcys]. The concentration of L-Hcys was varied at fixed concentrations of L-Ser (1.5 mM, O; 3.0 mM, $\square ; 6.0 \mathrm{mM}, \times ; 10 \mathrm{mM}, \Delta ; 20 \mathrm{mM}, \diamond ; 30 \mathrm{mM},+)$ and the fit of the entire data set to equation 2 is represented by the lines. The fitted parameters are given in Table 3. Reactions were in a total volume of $100 \mu \mathrm{L}$ at $25^{\circ} \mathrm{C}$ and were monitored at $340 \mathrm{~nm}$. Conditions: $50 \mathrm{mM}$ Tris (pH 8.6), $1.5 \mathrm{mM}$ NADH, $20 \mu \mathrm{M}$ PLP, 1.6 $\mu \mathrm{M} C B L$, and $1.4 \mu \mathrm{M} L D H$. Reactions were initiated by addition of $0.88 \mu \mathrm{M}$ hCBS. 


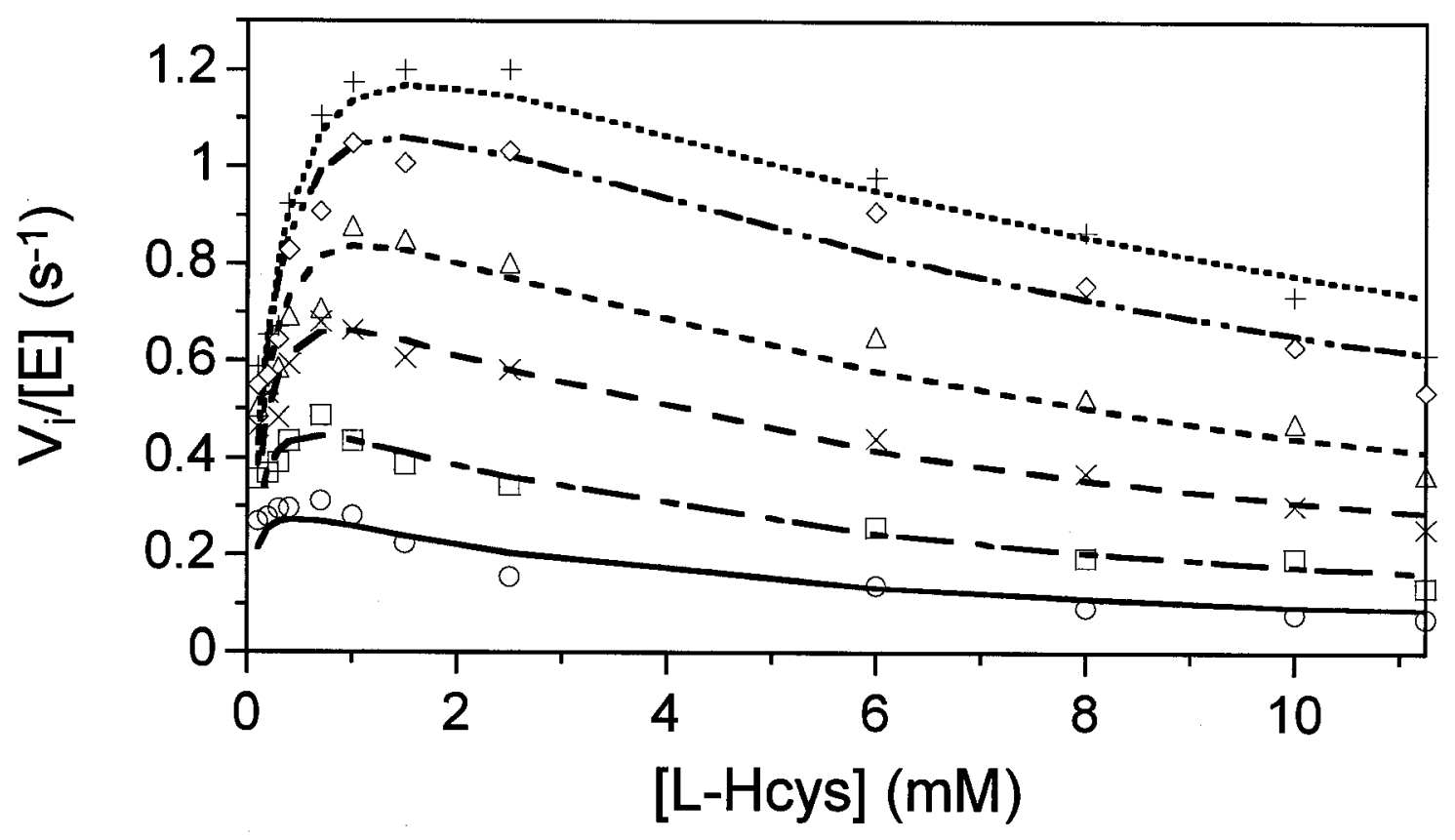




\subsection{Discussion.}

\subsubsection{Evaluation of hCBS expression constructs.}

The purification of hCBS from source is complicated by its tendency to aggregate and its susceptibility to proteolysis [Kraus and Roseburg, 1983]. Bukovska et al. [1998] developed a recombinant expression system, in which hCBS is expressed as a fusion protein with $\beta$-gal in $E$. coli, to circumvent this problem. However, proteolytic cleavage within the $E$. coli host cells was observed during expression of the $\beta$-gal/hCBS construct, thereby limiting the feasibility of affinity purification [Bukovska et al., 1998]. Two alternative systems were subsequently developed in which hCBS is expressed as a fusion protein with GST and cleaved with thrombin or Factor Xa protease [Shan and Kruger, 1998; Janosik et al., 2001a]. However, following protease cleavage of the GST/hCBS protein a 11- or 23-residue tag remains attached to hCBS, which was reported to alter the kinetic parameters of the enzyme [Frank et al., 2008]. Frank et al., [2008] effectively summarized the challenges involved in the purification of hCBS, from source or via recombinant expression, and developed a modified GST/hCBS expression construct that reduced the tag remaining at the $\mathrm{N}$-terminus of hCBS, following cleavage with PreScission protease, to a single glycine residue.

The three effective recombinant hCBS expression systems reported to date all rely upon expression of hCBS in fusion with GST and at least one step of affinity purification with glutathione-Sepharose affinity resin [Shan and Kruger, 1998; Janosik et al., 2001a; Frank et al., 2008; Taoka et al., 1998]. Therefore, in this study a 6-His tag was added to the $\mathrm{N}$-terminus of the GST/hCBS coding sequence, producing the 6-His-GST/hCBS construct, with the goal of reducing the cost of purification. The imidazole required for 
elution in Ni-NTA chromatography is $\sim 10-20$-fold less expensive than the glutathione used for elution/regeneration of glutathione-Sepharose affinity resin. However, although purification and thrombin cleavage of the 6-His-GST/hCBS enzyme was successful, the homogeneity of the hCBS produced was less than $90 \%$, as judged by SDS-PAGE, and the yield was $\sim 20$-fold lower than the $11.5 \mathrm{mg} / \mathrm{L}$ reported for the corresponding untagged GST/hCBS construct (Figure 12, Table 1) [Taoka et al., 1998]. This reduction in yield and purity may be due to the difference in plasmid and cell line used for expression; pTrc-99a in E. coli strain DH10b in this study $v$. the pGEX-4T1 vector in $E$. coli strain BL-21 employed by Taoka et al. [Shan and Kruger, 1998; Taoka et al., 1998].

To investigate the possibility that interaction with the glutathione $S$-transferase fusion partner may compound the tendency of the homotetrameric hCBS enzyme to aggregate, a new expression system was developed employing the green fluorescent protein (GFP), which has a similar subunit size as GST [Rufer et al., 2005; Yang et al., 1996]. The sequence of the linker between the GFP and hCBS proteins was designed to reduce the number of amino acids remaining at the amino-terminus of hCBS, following thrombin cleavage, to only three residues, and a 6-His tag was added to the aminoterminus of GFP to allow purification via Ni-NTA affinity chromatography. Although the purification of 6-His-GFP/hCBS was successful, the yield was $\sim 2$-3-fold lower (Table 1) than the 3-6 mg/L recently reported, for the GST-hCBS construct with the re-engineered linker region, by Frank et al. [2008]. The similar yields of the 6-His-GST/hCBS and 6His-GFP/hCBS expression constructs, 0.5 and $1.8 \mathrm{mg} / \mathrm{L}$, respectively (Table 1 ), suggest that the quaternary structure of GST or GFP does not impact the yield of soluble hCBS. 
All of the recombinant systems reported to date have relied upon expression of $\mathrm{hCBS}$ in fusion with a protein partner and require protease cleavage to release hCBS. Development of a purification procedure that eliminated the need for this step would result in savings in both cost and time. The effectiveness of the recently-described GST/hCBS expression construct, which incorporated a sufficiently long linker between the GST and hCBS fusion partners to allow independent folding [Frank et al., 2008], suggests that hCBS does not require the GST fusion partner to promote folding in E. coli. Therefore, expression constructs were designed in which only an N-terminal or Cterminal 6-His tag (Figure 11), in lieu of the $\beta$-gal, GST or GFP fusion partners employed to date, were added to enable Ni-NTA affinity chromatography. The hCBS/6-His construct, bearing the C-terminal affinity tag was insoluble. The carboxy-terminal $\sim 140$ residues of hCBS comprises the regulatory domain, removal of which was required to reduce aggregation in order to solve the crystal structure of the dimeric catalytic core of hCBS [Meier et al., 2001; Taoka et al., 1998]. Therefore, addition of an affinity tag to the regulatory domain of hCBS may destabilize its oligomeric structure, resulting in aggregation. In contrast, the 6-His/hCBS construct yielded enzyme of $>90 \%$ homogeneity with a single chromatographic step (Figure 12, Table 1). This is the first report of the expression and purification of recombinant hCBS from $E$. coli with an affinity tag instead of a protein fusion partner. An expression construct bearing a protease-cleavable, 6-His tag at the N-terminus (6-His-linker/hCBS) was also tested, as the ability to remove the affinity tag may be required for specific applications. Similar to the GST/hCBS construct described by Frank et al. [2008], which retains a single glycine residue following PreScission protease cleavage, 6-His-linker/hCBS is designed to retain only a single 
histidine residue at the amino-terminus after treatment with Factor Xa protease. The $>85 \%$ heme saturation of $6-\mathrm{His} / \mathrm{hCBS}$ and $6-\mathrm{His}-$ linker/hCBS are similar to that reported by Frank et al. [2008] for GST-hCBS and the yield is within $~ 2-3$-fold. Therefore, these constructs provide an alternative hCBS expression system with the advantages of decreased cost and time required for purification. When corrected by a factor of 2.4 , to compensate for the 12-degree temperature difference between the $25^{\circ} \mathrm{C}$ measurements described here (Table 2) and those reported at $37^{\circ} \mathrm{C}$, the $k_{\text {cat }}$ of 6-His/hCBS $\left(\sim 2.1 \mathrm{~s}^{-1}\right)$ and 6-His-linker/hCBS $\left(\sim 3.1 \mathrm{~s}^{-1}\right)$ are similar to the values of $2.8 \mathrm{~s}^{-1}\left(V_{\max }=159 \mu \mathrm{mol} / \mathrm{h} / \mathrm{mg}\right)$ and 3.7-4.7 $\mathrm{s}^{-1}$ reported by Taoka et al. [1998] and by Frank et al. [2008], respectively, for different GST/hCBS constructs. The kinetic parameters in Table 2 were determined under conditions of saturating one substrate while varying the other. Although this method does not allow for consideration of substrate inhibition, and can therefore result in the underestimation of $k_{\text {cat }}$, it was selected for comparison of kinetic parameters with reported values, which have been consistently determined in this manner for hCBS to date. The kinetic parameters of the 6-His-linker/hCBS enzyme are not altered by removal of the affinity tag and linker (Figure 12, Table 2). Therefore, the reduction of $K_{m}{ }^{L \text {-Hcys }}$ observed for the GST-hCBS construct of Janosik et al. [2001b] that retains a 23-residue $\mathrm{N}$-terminal extension following proteolytic cleavage, compared to that expressed from the $\beta$-gal/hCBS construct [Bukovska et al., 1994; Frank et al., 2008] is not observed for 6-His-linker/hCBS. 


\subsubsection{Steady-state characterization of $h C B S$.}

The continuous CBS assay, which relies on CBL and LDH as coupling enzymes, was employed for the first time in this study to determine the steady-state kinetic parameters of $\mathrm{hCBS}$. Reports of kinetic parameters for $\mathrm{hCBS}$ show a wide range of variation. For example, reported values of $K_{m}{ }^{L-S e r}, K_{m}{ }^{L-H c y s}$ and $k_{c a t}$ range between $1.15-$ $4.9 \mathrm{mM}, 0.17-7.17 \mathrm{mM}$ and $3.5-6.2 \mathrm{~s}^{-1}$, respectively [Janosik et al., 2001b; Kraus et al., 1978; Bukovska et al., 1994; Frank et al., 2008; Taoka et al., 1998]. While the use of various expression systems may be a contributing factor in the large variation in reported kinetic parameters, the ${ }^{14} \mathrm{C}-\mathrm{L}-\mathrm{Ser}$ endpoint assay employed to measure hCBS activity also merits consideration. The laborious nature of this assay has precluded the collection of large data sets necessary for the global analysis required for a bi-substrate system. Therefore, all kinetic parameters reported have been under the assumption of saturating concentration of either L-Ser or L-Hcys and the data fit to the Michaelis-Menten equation. Substrate inhibition has not been previously reported for the wild-type human enzyme, although yeast CBS is known to be similarly inhibited at elevated L-Hcys concentration [Jhee et al., 2000b].

Frank et al. [2008] determined the steady-state kinetic parameters of hCBS, bearing only an amino-terminal glycine residue following proteolytic cleavage form GST, using the ${ }^{14} \mathrm{C}$-L-Ser-based endpoint assay at $37^{\circ} \mathrm{C}$, and reported $K_{m}{ }^{L-H c y s}$ and $K_{m}{ }^{L-S e r}$ values of $1.04 \pm 0.24 \mathrm{mM}$ and $1.41 \pm 0.35 \mathrm{mM}$, respectively. Although the $K_{m}{ }^{L-S e r}$ and $K_{m}{ }^{\mathrm{L}-\mathrm{Hcys}}$ of 6-His/hCBS and 6-His-linker/hCBS are $\sim 5$-fold greater and $\sim 3-5$-fold lower, respectively (Table 3), than those reported by Frank et al. [2008], they are within the broad range of values reported for hCBS $\left(K_{m}{ }^{L-S e r}=1.15-4.9 \mathrm{mM}\right.$ and $K_{m}{ }^{L-H c y s}=0.17-$ 
7.17 mM) [Janosik et al., 2001b; Kraus et al., 1978; Bukovska et al., 1994; Frank et al., 2008; Taoka et al., 1998]. When corrected by a factor of 2.4 , to compensate for the $12-$ degree temperature difference between the $25^{\circ} \mathrm{C}$ measurements described here (Table 2) and those reported at $37^{\circ} \mathrm{C}$, the value of $4.0 \mathrm{~s}^{-1}$ for the $k_{c a t}$ of both 6-His/hCBS and 6-Hislinker/hCBS (Table 9.3) is not significantly different from the distinct values of $k_{c a t}{ }^{L-H c y s}$ $\left(4.66 \pm 0.67 \mathrm{~s}^{-1}\right)$ and $k_{c a t}^{L-S e r}\left(3.67 \pm 0.67 \mathrm{~s}^{-1}\right)$ reported by Frank et al. [2008]. The ability of hCBS to catalyze the reverse-physiological hydrolysis of L-Cth was also investigated (Table 3) and the $k_{\text {cat }}$ and $K_{m}{ }^{L-C t h}$ values are 5-fold and 2-fold lower, respectively, than the corresponding values for the model yeast enzyme [Aitken and Kirsch, 2003]. Similar to $\mathrm{yCBS}$, the $k_{\text {cat }}$ of the hCBS-catalyzed condensation reaction is $\sim 70$-fold greater than that of the L-Cth hydrolysis reaction.

In summary, the 6-His/hCBS and 6-His-linker/hCBS constructs provide costeffective and efficient expression systems for the purification and subsequent kinetic studies of wild type and mutants of hCBS. Characterization of hCBS expressed from two constructs demonstrated that, similar to the yeast enzyme, hCBS is substrate inhibited by L-Hcys and has the ability to catalyze both the physiological reaction in which L-Ser and L-Hcys are condensed to form L-Cth and the reverse-physiological hydrolysis of L-Cth. 
10. Characterization of S289A,D Mutants of Yeast Cystathionine $\beta$-Synthase. 


\subsection{Abstract.}

Cystathionine $\beta$-synthase (CBS) catalyzes the pyridoxal 5'-phosphate (PLP)dependent condensation of L-serine and L-homocysteine to form L-cystathionine in the first step of the reverse transsulfuration pathway. Residue S289 of yeast CBS, predicted to form a hydrogen bond with the pyridine nitrogen of the PLP cofactor, was mutated to alanine and aspartate. The $k_{c a t} / K_{m}{ }^{L-S e r}$ of the S289A mutant is reduced by a factor of $\sim 800$ and the $\beta$-replacement activity of the S289D mutant is undetectable. Fluorescence energy transfer between tryptophan residue(s) of the enzyme and the PLP cofactor, observed in the wild-type enzyme and diminished in the S289A mutant, is absent in S289D. These results demonstrate that residue $\mathbf{S} 289$ is essential in maintaining the properties and orientation of the pyridine ring of the PLP cofactor. The reduction in activity of ytCBSS289A suggests that ytCBS catalyzes the $\alpha, \beta$-elimination of L-Ser via an $\mathrm{E}_{1}$ mechanism. 


\subsection{Introduction.}

Cystathionine $\beta$-synthase (CBS; E.C. 4.2.1.22.) catalyzes the condensation of Lserine (L-Ser) and L-homocysteine (L-Hcys) to form L-cystathionine (L-Cth) [Borcsok and Abeles, 1982]. The enzymes tryptophan synthase $(\operatorname{TrpS})$ and $O$-acetylserine sulfhydralase (OASS), also members of the $\beta$-subfamily of PLP-dependent enzymes, catalyze similar $\beta$-replacement reactions in the synthesis of tryptophan and cysteine, respectively [Cook and Wedding, 1976; Miles, 2001; Mehta and Christen, 2000]. The first half reaction of these enzymes is an $\alpha, \beta$-elimination that consists of abstraction of the $\mathrm{C}_{\alpha}$ proton and $\beta$ elimination of the acetate moiety of $O$-acetyl-L-serine (OAS) by OASS or the hydroxyl group of L-Ser by TrpS and CBS, resulting in formation of PLP-bound aminoacrylate. The reaction of this intermediate with $\mathrm{H}_{2} \mathrm{~S}$, indole or L-Hcys comprises that second half reaction of OASS, TrpS and CBS, respectively [Cook and Wedding, 1976; Miles, 2001; Jhee et al., 2000a].

While $\alpha, \beta$-elimination reactions may proceed by either an $E_{1}$ or $E_{2}$ mechanism, depending on the nature of the leaving group, those dependent on pyridoxal 5'-phosphate (PLP) are generally thought to follow an $E_{1}$ mechanism in which the carbanion formed upon abstraction of the $C_{\alpha}$ proton is resonance stabilized in the quinonoid intermediate [Dunathan, 1971; Saunders, 1976; Tai and Cook, 2001]. This is exemplified by the reaction catalyzed by the $\beta$-subunit of $\operatorname{TrpS}$, in which $\beta$-elimination of the hydroxyl group of L-Ser occurs in a stepwise manner [Drewe and Dunn, 1985; Jhee et al., 1998b]. In contrast, no quinonoid intermediate is observed during the $\beta$-elimination of acetate from OAS by OASS, and a concerted $\mathrm{E}_{2}$ mechanism has been demonstrated for this reaction (Figure 14) [Tai and Cook, 2001; Daum et al., 2003]. 
The interactions between active-site residues and the PLP cofactor modify the electronic properties of the latter and maintain its catalytically-productive orientation in the active site [Eliot and Kirsch, 2004]. The pyridine nitrogen of the PLP ring is within $2.9,2.8$ and $3.0 \AA$ of the oxygen atom of the hydroxyl side chain of an active-site serine residue in TrpS (S377), OASS (S272) and human CBS (S349, which corresponds to S289 of yeast CBS), respectively [Hyde et al., 1988; Meier et al., 2001; Burkhard et al., 1998]. However, while the structurally-conserved S349 and S272, of human CBS (hCBS) and OASS, respectively, are located at the si face of the cofactor, S377 of TrpS is structurally distinct and situated at the re face [Hyde et al., 1988; Burkhard et al.,1998; Taoka et al., 2002]. The transient quinonoid intermediate of $\operatorname{TrpS}$ is stabilized under equilibrium conditions and the $\mathrm{p} K_{a}$ of the imine nitrogen of the internal aldimine, which is $\mathrm{pH}$ independent in the wild-type enzyme, is shifted to $\sim 7.7$ in the S377D mutant of this enzyme [Jhee et al., 1998a]. The near-native catalytic efficiency of the OASS-S272A,D mutants, contrasts with the drastic reduction in the activity of the corresponding S377A,D mutants of TrpS, providing further evidence of the $\mathrm{E}_{2}$ mechanism of OASS [Jhee et al., 1998a; Daum et al., 2003].

The reaction catalyzed by CBS shares distinct features with those of $\operatorname{TrpS}$ and OASS, but also has several unique characteristics. For example, only a transient geminal diamine and the external aldimine of aminoacrylate are observed upon reaction of yeast CBS (yCBS) with L-Ser (Figure 14) [Jhee et al., 2001]. In contrast with TrpS and OASS, the external aldimine of the L-Ser substrate is not detected and there is no primary isotope effect for abstraction of the $\mathrm{C}_{\alpha}$ proton by yCBS [Drewe et al., 1985; Jhee et al., 2001; Woehl et al., 1996]. This, in combination with the lack of a detectable quinonoid 
intermediate for yCBS, in contrast with $\operatorname{TrpS}$, suggests that either the quinonoid does not accumulate or that abstraction of the $C_{\alpha}$ proton and $\beta$-elimination of the hydroxyl leaving group are concerted, as in OASS [Jhee et al., 2001; Cook, 2003].Although, the CBScatalyzed $\beta$-elimination of hydroxide from L-Ser likely occurs via the $\mathrm{E}_{1}$ mechanism of TrpS, residue S289 of yCBS is structurally conserved with S272 of OASS and neither yCBS or OASS form an observable quinonoid intermediate under presteady-state conditions [Tai and Cook, 2001; Jhee et al., 2001; Woehl et al., 1996]. Therefore, the S289A,D site-directed mutants were constructed to probe the mechanism of yCBS. The yeast enzyme is employed as it lacks the heme prosthetic group of hCBS, which masks the absorbance of the PLP cofactor and precludes pre-steady state investigations [Jhee et $a l ., 2000 \mathrm{~b}]$. Analysis of the S289A,D mutants suggests that, like TrpS, the $\alpha, \beta-$ elimination catalyzed by yCBS follows an $\mathrm{E}_{1}$ mechanism. 
Figure 14. Proposed mechanism of the $\alpha, \beta$-elimination half reaction of ytCBS demonstrating possible $E_{1}$ and $E_{2}$ reaction pathways, which are employed by $\operatorname{TrpS}$ and OASS, respectively [Jhee et al., 2001, Cook, 2003]. The labeled intermediates correspond to the (1) internal aldimine (412 nm), (2) geminal diamine (320-340 nm), (3) external aldimine of L-Ser $(410-420 \mathrm{~nm}),(4)$ quinonoid $(480 \mathrm{~nm})$ and $(5)$ external aldimine of aminoacrylate ( 320 and $460 \mathrm{~nm})$ [Jhee et al., 2001]. 

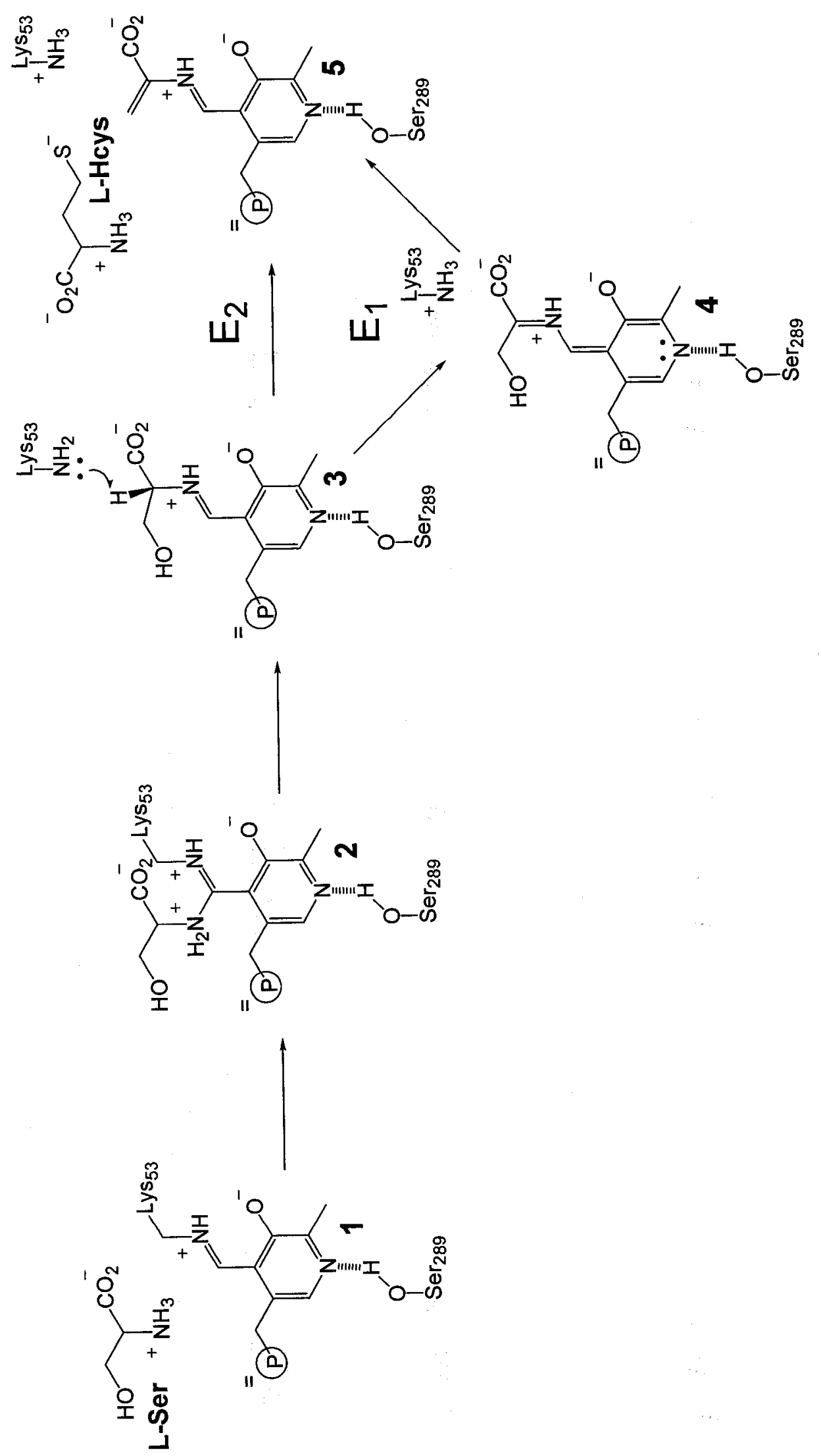


\subsection{Methods.}

\subsubsection{Reagents.}

L-Cth [S-(2-amino-2-carboxyethyl)-L-homocysteine], $\beta$-NADH ( $\beta$-nicotinamide adenine dinucleotide, reduced form), L-Ser and L-Hcys thiolactone were purchased from Sigma. Dithiothreitol (DTT), ampicillin, 5,5'-dithio-bis-(2-nitrobenzoic acid) (DTNB) and imidazole were obtained from Fisher Scientific and L-lactate dehydrogenase (LDH) and protease inhibitor (Complete EDTA-free) tablets were from Roche Diagnostics. Restriction endonucleases and T4 DNA ligase were from New England Biolabs. Nickelnitrilotriacetic acid (Ni-NTA) resin was from Qiagen. L-Hcys was prepared from the thiolactone according to the method of Kashiwamata and Greenberg [Kashiwamata and Greenberg, 1970]. Cystathionine $\beta$-lyase (CBL) was expressed and purified as described previously [Aitken and Kirsch, 2003].

\subsubsection{Construction, Expression and Purification of ytCBS-His mutants.}

The S289A and S289D site-directed mutants were constructed by Dr. Susan Aitken. Overlap-extension polymerase chain reaction (PCR), with the pSECseq1 (CGG TTC TGG CAA ATA TTC TGA AAT GAG CTG) pSECseq7r (GCC CGC CAC CCT CCG GGC CGT TGC TTC GC) flanking primers and either the S289Af (CTT GGT GGG TGG TGC CTC CGG TTC TGC CTT CAC) or S289Df(CTT GGT GGG TGG TGA TTC CGG TTC TGC CTT CAC) mutagenic primers and their reverse complements, was employed to introduce the S289A and S289D site-directed mutations in the pTSECb-His plasmid, which contains the gene encoding the truncated yCBS 
(ytCBS; residues 1-353), lacking the regulatory domain, with a C-terminal, 6-His affinity tag [Aitken and Kirsch, 2004; Higuchi, 1990]. The amplification product was digested with BamHI and PstI, inserted at the corresponding sites of the pT-SECb-His vector and transformed into the Escherichia coli strain DH10B (Gibco BRL) via electroporation (Gene Pulser, BioRad). The S289A and S289D site-directed mutants were expressed and purified via Ni-NTA affinity chromatography (Qiagen), as previously described for the heterologous expression of 6-His tagged ytCBS [Aitken and Kirsch, 2004].

\subsubsection{Steady-State Kinetics.}

Enzyme activity was measured in a total volume of $1 \mathrm{~mL}$ at $37^{\circ} \mathrm{C}$ with a model HP8453 spectrophotometer (Agilent). The assay buffer comprised $50 \mathrm{mM}$ Tris, $\mathrm{pH} 8.6$ and $20 \mu \mathrm{M}$ PLP. A background rate, for all components except the ytCBS enzyme, was recorded for each sample before initiating the reaction by the addition of ytCBS. Data was fit by nonlinear regression with the program SAS (SAS Institute, Cary, NC). The continuous CBL-LDH assay, in which formation of L-Cth is detected continuously, was employed to monitor the ytCBS $\beta$-replacement activity. The $\beta$-replacement assay was done by Dr. Aitken. The LDH and 5,5'-Dithiobis-(2-Nitrobenzoic Acid) (DTNB) assays were employed to monitor the $\beta$-elimination of $\mathrm{L}$-Ser, producing pyruvate and $\mathrm{NH}_{3}$, and the hydrolysis of L-Cth to L-Ser and L-Hcys, respectively [Aitken and Kirsch, 2003; Aitken and Krisch, 2004]. The $k_{c a t E}$ and $K_{m E}{ }^{L-S e r}$ and the $k_{c a t R}$ and $K_{m R}{ }^{L-C t h}$ for the $\beta$ elimination and the L-Cth hydrolysis reactions, respectively, were determined from the fit of the data to the Michaelis-Menten equation. The $\mathrm{E}$ and $\mathrm{R}$ subscripts denote the $\beta$ - 
elimination and reverse-physiological reactions, respectively. The $k_{c a t} / K_{m}$ for each reaction was obtained independently from equation 3.

$$
\frac{v}{[E]}=\frac{k_{c a t} / K_{m} \times[L-C t h]}{1+\left[L-C t h / K_{m}\right.}
$$

The kinetic parameters of the $\beta$-replacement reaction were determined from the fit of the data to equation 4 , where the $\mathrm{F}$ subscript denotes the physiological, $\beta$-replacement activity [Aitken and Kirsch, 2004; Jhee et al., 2000b]. Equation 4 was rearranged by dividing all terms by $K_{m F}{ }^{L-S e r}$ or $K_{m F}{ }^{L-H c y s}$ to obtain independent values for $k_{c a t F} / K_{m F}{ }^{L-S e r}$ and $k_{c a t F} / K_{m F}^{L-H c y s}$, respectively.

$$
\frac{v}{[\mathrm{E}]}=\frac{k_{\text {catF }}[\mathrm{L}-\mathrm{Ser}[\mathrm{L}-\mathrm{Hcys}]}{K_{\mathrm{mF}}^{\mathrm{L}-\mathrm{Hcys}}[\mathrm{L}-\mathrm{Ser}]+K_{\mathrm{mF}}^{\mathrm{L}-\mathrm{Ser}}\left[\mathrm{L}-\mathrm{Hcys}\left(1+\frac{[\mathrm{L}-\mathrm{Hcys}]}{K_{\mathrm{iF} 1}^{\mathrm{L}-\mathrm{Hcys}}}\right)+[\mathrm{L}-\mathrm{Ser}]\left[\mathrm{L}-\mathrm{Hcys}\left(1+\frac{[\mathrm{L}-\mathrm{Hcys}]}{K_{\mathrm{iF} 2}^{\mathrm{L}-H c y s}}\right)\right.\right.}
$$

\subsubsection{Fluorescence Spectroscopy.}

Fluorescence spectra were acquired with a Cary Eclipse spectrofluorimeter (Varian) at $37^{\circ} \mathrm{C}$ in $50 \mathrm{mM}$ Tris, $\mathrm{pH}$ 8.6. To investigate the possibility of energy transfer from one or more of the four tryptophan residues of ytCBS to the PLP cofactor of ytCBS the fluorescence spectrum $\left(\lambda_{\mathrm{ex}}=298 \mathrm{~nm}\right)$ of $20 \mu \mathrm{M}$ enzyme was recorded from $320-650$ $\mathrm{nm}$ at $2 \mathrm{~nm}$ resolution with excitation and emission slit widths of $8 \mathrm{~nm}$ in the absence and 
presence of $50 \mathrm{mM} \mathrm{L-Ser} \mathrm{[McClure} \mathrm{and} \mathrm{Cook,} \mathrm{1994].} \mathrm{The} \mathrm{apparent} \mathrm{dissociation} \mathrm{constant}$ for the E-AA complex due to L-Ser association with free enzyme $\left(K_{d(a p p)}^{L-S e r}\right)$ was determined by the protocol described by Jhee et al. [2000b], where a $1.0 \mu \mathrm{M}$ solution of ytCBS enzyme was titrated with aliquots of L-Ser, and the increase in fluorescence at 540 $\mathrm{nm}\left(\lambda_{\mathrm{ex}}=460 \mathrm{~nm}\right)$, due to formation of the aminoacrylate (AA) intermediate, was monitored. The change in fluorescence at $540 \mathrm{~nm}$ was plotted versus [L-Ser] and fit to equation 5 [Jhee et al., 2000b].

$$
\Delta F=\frac{\Delta F_{\max }[L-S e r]}{K_{d(a p p)}^{L-S e r}+[L-S e r]}
$$

The method of Adams [1969] was adapted for determination of the PLP saturation of wild-type and mutant enzymes. The enzymes were diluted (to $0.8-1.2 \mu \mathrm{M}$ ) in $200 \mu \mathrm{L}$ in $5 \mathrm{mM}$ phosphate buffer, $\mathrm{pH}$ 7.4. Following the addition of an equal volume of $11 \%$ TCA and incubation for $15 \mathrm{~min}$ at $50^{\circ} \mathrm{C}, 140 \mu \mathrm{L}$ of $3.3 \mathrm{M} \mathrm{K}_{2} \mathrm{HPO}_{4}$ and $50 \mu \mathrm{L}$ of $0.02 \mathrm{M}$ $\mathrm{KCN}$ were added and samples incubated at $50^{\circ} \mathrm{C}$ for a further $25 \mathrm{~min}$ prior to the addition of $70 \mu \mathrm{L}$ of $28 \% \mathrm{H}_{3} \mathrm{PO}_{4}$ and $640 \mu \mathrm{L}$ of $2 \mathrm{M}$ potassium acetate, $\mathrm{pH}$ 3.8. The PLP concentration of the samples was determined from comparison of the fluorescence intensity at $425 \mathrm{~nm}\left(\lambda_{\mathrm{ex}}=325 \mathrm{~nm}\right)$ to a standard curve of 0-20 $\mu \mathrm{M}$ PLP. The PLP saturation of each enzyme was determined from the ratio of PLP concentration to the concentration of enzyme monomer.

\subsubsection{Single Turnover Measurements.}

The reaction of $20 \mu \mathrm{M}$ S289D with $150 \mathrm{mM} \mathrm{L-Ser}$ in $50 \mathrm{mM}$ Tris, $\mathrm{pH} 8.6$, at 25 
${ }^{\circ} \mathrm{C}$ was monitored for formation of the external aldimine of aminoacrylate at 320 and 460 $\mathrm{nm}$ for $40 \mathrm{~min}$ [Jhee et al., 2001]. The reaction of $20 \mu \mathrm{M}$ S289A with 5-200 mM L-Ser was monitored at $320 \mathrm{~nm}$ and $460 \mathrm{~nm}$, under the same conditions, and the $460 \mathrm{~nm}$ data were fit to equation 6 to obtain $k_{o b s}$ for the formation of the aminoacrylate intermediate at each concentration of L-Ser.

$$
A_{460}=A_{\min }+\Delta A e^{-k_{o b s} t}
$$

\subsubsection{Spectrophotometric Titration of the ytCBS Internal Aldimine.}

The experimental data for the $\mathrm{pH}$ titration study was obtained from Dr. Aitken. The internal aldimine of wild-type ytCBS and the S289A and S289D site-directed mutants were titrated versus $\mathrm{pH}$. The $\mathrm{pH}$ of a solution of $20 \mu \mathrm{M}$ enzyme in 5 mM TAPS ( $\mathrm{p} K_{\mathrm{a}} 8.4, \mathrm{pH} 7.2$ ), containing $0.5 \mathrm{M} \mathrm{KCl}$, was varied by successive additions of $0.5 \mathrm{M}$ AMPSO (pK $K_{\mathrm{a}}$ 9.0, $\left.\mathrm{pH} 10.6\right)$ below $\mathrm{pH} 9.0 ; 0.5 \mathrm{M}$ CAPS $\left(\mathrm{p} K_{\mathrm{a}} 10.4, \mathrm{pH} 11.5\right)$ between $\mathrm{pH}$ 9.0- 10.5; and $1.0 \mathrm{M} \mathrm{NaOH}$ above $\mathrm{pH} 10.5$. The enzyme solution was drawn through a $0.2-\mu \mathrm{m}$ filter to reduce light scattering from precipitate, and the $\mathrm{pH}$ of the solution was determined prior to each absorbance measurement. Spectra were recorded from 250 to $500 \mathrm{~nm}$ on a UVIKON 360 double-beam spectrophotometer (KONTRON Instruments). 


\subsection{Results.}

\subsubsection{Steady-state kinetic parameters.}

The protein expression levels of the S289A (77 mg/L) and S289D (78 mg/L) sitedirected mutants were comparable to wild-type ytCBS (36 mg/L). The $\beta$-replacement activity of ytCBS-S289D is undetectable and the $k_{c a t F} / K_{m F}{ }^{L-S e r}$ of the S289A mutant is

reduced by $\sim 800$-fold, compared to the wild type enzyme (Table 4$)$. The $K_{d(a p p)}{ }^{L-S e r}$ value of S289A is the same as wild-type, suggesting that the L-Ser binding site of this mutant is unperturbed. The lack of aminoacrylate formation precluded measurement of $K_{d(a p p)}{ }^{L-S e r}$ for S289D (Table 4). The $K_{m F}^{L-H c y s}$ of S289A is unchanged, while the $K_{m F}{ }^{L-S e r}$ is increased $\sim 40$-fold. The reverse-physiological L-Cth hydrolysis reaction is undetectable for S289D and the $\sim 1400$-fold reduction of $k_{\text {catR }} / K_{m R}^{L-C t h}$ for S289A is dominated by a 230-fold decrease in $k_{c a t R}$. Although the S289A mutant possesses a marginal $\beta$-elimination activity $\left(k_{\text {catE }} / K_{m E}^{L-S e r}=0.11 \mathrm{M}^{-1} \mathrm{~s}^{-1}\right)$, the S289D mutant, like the wild-type enzyme, does not catalyze the conversion of L-Ser to pyruvate and ammonia (Table 4).

\subsubsection{Formation and stability of the aminoacrylate intermediate.}

The degree of PLP saturation of the wild-type, S289A and S289D enzymes is $96 \%, 44 \%$ and $41 \%$, respectively, demonstrating that binding of the cofactor to the mutants is impaired, but not precluded. Formation of the aminoacrylate intermediate is not observed for the S289D mutant at L-Ser concentrations up to $150 \mathrm{mM}$. Unlike the S377D mutant of TrpS, for which a stable quinonoid is formed under equilibrium conditions following reaction with L-Ser, no quinonoid is detected for the corresponding 
ytCBS-S289D. Addition of 5-150 mM L-Ser to the S289A mutant, results in a decrease in absorbance at $412 \mathrm{~nm}$, corresponding to the internal aldimine, with a concomitant increase in intensity at 460 and $320 \mathrm{~nm}$ consistent with formation of the external aldimine of aminoacrylate (Figures 14 and 15) [Jhee et al., 2001]. The rate of formation of the aminoacrylate intermediate is drastically reduced in the S289A mutant, compared to wild-type ytCBS (Figure 15). As reported by Jhee et al. [2001], the reaction of free ytCBS enzyme (E) with L-Ser to form AA follows the sequence of equation 7,

$$
E+L-\operatorname{Ser} \underset{k_{2}}{\stackrel{k_{1}}{\Leftrightarrow}} E \bullet L-\operatorname{Ser} \underset{k_{4^{*}}}{\stackrel{k_{3}}{\Leftrightarrow}} E-A A
$$

where $k_{4^{*}}=k_{4}\left[\mathrm{H}_{2} \mathrm{O}\right]$ and $K_{21}=k_{2} / k_{1}$. The rate $\left(k_{o b s}\right)$ of AA formation of S289A at $25{ }^{\circ} \mathrm{C}$ was determined (eq 6) at 5-150 mM L-Ser and the resulting $k_{o b s}$ values were plotted $v s$. LSer concentration (Figure 15) and fit to equation 8 [Jhee et al., 2001].

$$
k_{o b s}=\frac{k_{3}[\mathrm{~L}-\mathrm{Ser}]}{K_{21}+[\mathrm{L}-\mathrm{Ser}]}+k_{4^{*}}
$$

The values of $k_{3}\left((1.3 \pm 0.1) \times 10^{-3} \mathrm{~s}^{-1}\right)$ and $K_{21}(25 \pm 0.4 \mathrm{mM})$ for S289A are reduced approximately $1.4 \times 10^{-5}$ and 5-fold, respectively, compared to those reported at for the wild-type enzyme $\left(k_{3}=177 \pm 8 \mathrm{~s}^{-1}\right.$ and $\left.K_{21}=4.6 \pm 0.8 \mathrm{mM}\right)$ at $25^{\circ} \mathrm{C}$ [Jhee et al., 2001]. The aminoacrylate intermediate of both wild-type ytCBS and the S289A mutant is stable over $360 \mathrm{~min}$ at $\mathrm{pH} 8.6$ (Figure 16) and 10.5. This contrasts with the aminoacrylate of OASS, and the corresponding S272A mutant, which decays almost completely during the same period and for which the rate of decay is increased at $\mathrm{pH}>7.5$ [Daum et al., 2003]. 


\subsubsection{Equilibrium spectroscopic studies.}

Absorbance spectra of the S289A and S289D site-directed mutants were recorded over the $\mathrm{pH}$ range of 7.1-12.3. Similar to the wild-type enzyme, the $412 \mathrm{~nm}$ absorbance of the internal aldimine of S289A and S289D mutants, is $\mathrm{pH}$ independent below $\mathrm{pH} 11$ [Aitken and Kirsch, 2003]. Fluorescence spectra $\left(\lambda_{\mathrm{ex}}=298 \mathrm{~nm}\right.$ and $\left.\lambda_{\mathrm{em}}=320-650 \mathrm{~nm}\right)$ of the wild-type and mutant enzymes were recorded in the absence and presence of $20 \mathrm{mM}$ L-Ser (Figure 16). Upon excitation at $298 \mathrm{~nm}$, a peak is observed at $\sim 500 \mathrm{~nm}$ in the emission spectrum of the wild type enzyme that is likely the result of Forster energy transfer from tryptophan residue(s) of the enzyme to the PLP cofactor. A similar effect has been observed for OASS, in which the residue was identified as W162 [McClure and Cook, 1996]. In contrast, while the $\sim 500$-nm peak of the S289A mutant is less pronounced than the wild-type (Figure 16) enzyme, it is not detectable for the S289D mutant, suggesting that the orientation of the cofactor is altered as a result of these mutations. Addition of L-Ser to the wild type and S289A enzymes results in a shift in the fluorescence emission to $540 \mathrm{~nm}$, corresponding to the external aldimine of aminoacrylate, and a decrease in its intensity (Figure 16). 
Table 4. Steady-state kinetic parameters for $\mathrm{ytCBS}^{a}$.

\begin{tabular}{|c|c|c|}
\hline & $\mathrm{ytCBS}^{b}$ & $\mathrm{~S} 289 \mathrm{~A}^{c}$ \\
\hline \multicolumn{3}{|c|}{ L-serine + L-homocysteine $\rightarrow$ L-cystathionine } \\
\hline $\mathrm{k}_{\mathrm{catF}}\left(\mathrm{s}^{-1}\right)$ & $17 \pm 1$ & $0.85 \pm 0.08$ \\
\hline $\mathrm{K}_{\mathrm{mF}}^{\mathrm{L}-\mathrm{Ser}}(\mathrm{mM})$ & $0.7 \pm 0.2$ & $27.1 \pm 5.1$ \\
\hline $\mathrm{K}_{\mathrm{mF}}^{\mathrm{L}-\mathrm{Hcys}}(\mathrm{mM})$ & $0.21 \pm 0.04$ & $0.16 \pm 0.03$ \\
\hline $\mathrm{k}_{\mathrm{catF}} / \mathrm{K}_{\mathrm{mF}}^{\mathrm{L}-\mathrm{Ser}}\left(\mathrm{M}^{-1} \mathrm{~s}^{-1}\right)$ & $(2.5 \pm 0.6) \times 10^{4}$ & $31 \pm 4$ \\
\hline $\mathrm{k}_{\mathrm{catF}} / \mathrm{K}_{\mathrm{mF}}{ }^{\mathrm{L}-\mathrm{Hcys}}\left(\mathrm{M}^{-1} \mathrm{~s}^{-1}\right)$ & $(8 \pm 1) \times 10^{4}$ & $(6.3 \pm 0.9) \times 10^{3}$ \\
\hline $\mathrm{K}_{\mathrm{iF1}}{ }^{\mathrm{L}-\mathrm{Hcys}}(\mathrm{mM})$ & $1.0 \pm 0.4$ & $2.9 \pm 1.3$ \\
\hline $\mathrm{K}_{\mathrm{iF} 2}{ }^{\mathrm{L}-\mathrm{H} \text { cys }}(\mathrm{mM})$ & $15 \pm 7$ & $3.6 \pm 1.2$ \\
\hline $\mathrm{K}_{\mathrm{d}(\mathrm{app})^{\mathrm{L}-\mathrm{Ser}}(\mathrm{mM})^{d}}$ & $15.1 \pm 0.6$ & $13.6 \pm 0.2$ \\
\hline \multicolumn{3}{|c|}{ L-serine $\rightarrow$ pyruvate $+\mathrm{NH}_{3}$} \\
\hline $\mathrm{k}_{\mathrm{catE}}{ }^{\mathrm{L}-\mathrm{Ser}}\left(\mathrm{s}^{-1}\right)$ & n.d. & $0.0061 \pm 0.0002$ \\
\hline $\mathrm{K}_{\mathrm{mE}}{ }^{\mathrm{L}-\mathrm{Ser}}(\mathrm{mM})$ & n.d. & $56.4 \pm 4.5$ \\
\hline $\mathrm{k}_{\mathrm{catE}} / \mathrm{K}_{\mathrm{mE}}{ }^{\mathrm{L}-\mathrm{Ser}}\left(\mathrm{M}^{-1} \mathrm{~s}^{-1}\right)$ & n.d. & $0.11 \pm 0.06$ \\
\hline \multicolumn{3}{|c|}{ L-cystathionine $\rightarrow$ L-homocysteine + L-serine } \\
\hline $\mathrm{k}_{\mathrm{catR}}\left(\mathrm{s}^{-1}\right)$ & $1.03 \pm 0.02$ & $0.0045 \pm 0.0002$ \\
\hline $\mathrm{K}_{\mathrm{mR}}^{\mathrm{L}-\mathrm{CCth}}(\mathrm{mM})$ & $0.14 \pm 0.01$ & $0.9 \pm 0.1$ \\
\hline $\mathrm{k}_{\mathrm{catR}} / \mathrm{K}_{\mathrm{mR}}{ }^{\mathrm{L}-\mathrm{Cth}}\left(\mathrm{M}^{-1} \mathrm{~s}^{-1}\right)$ & $(7.5 \pm 0.4) \times 10^{3}$ & $5.3 \pm 0.7$ \\
\hline
\end{tabular}

${ }^{a}$ Kinetic parameters for the $\beta$-replacement, $\beta$-elimination and $\mathrm{L}$-Cth hydrolysis activities are denoted by the subscripts F, E and R, respectively. Kinetic measurements were carried out in $50 \mathrm{mM}$ Tris, $\mathrm{pH} 8.6$, containing $20 \mu \mathrm{M}$ PLP at $37^{\circ} \mathrm{C}$. n.d. denotes that activity was not detectable.

${ }^{b}$ From reference [Aitken and Kirsch, 2004].

${ }^{c} \beta$-replacement conditions: $0.4 \mu \mathrm{M}$ CBL, $1.4 \mu \mathrm{M} \mathrm{LDH}, 200 \mu \mathrm{M}$ NADH, $0.03-8.8 \mathrm{mM} \mathrm{L}-$ Hcys, $0.25-100 \mathrm{mM} \mathrm{L}-\mathrm{Ser}$, and 9.4-18.8 $\mu \mathrm{M}$ S289A. Data were fit to eq 4. $\beta$-elimination conditions: $300 \mu \mathrm{M}$ NADH, $2.3 \mu \mathrm{M} \mathrm{LDH}, 2.7-270 \mathrm{mM}$ L-Ser and $13 \mu \mathrm{M}$ S289A. Hydrolysis of L-Cth to L-Ser and L-Hcys conditions: $2 \mathrm{mM} \mathrm{DTNB}, 0.01-6.3 \mathrm{mM} \mathrm{L}-\mathrm{Cth}$ and $27.4 \mu \mathrm{M}$ S289A. $\beta$-elimination and L-Cth hydrolysis data were fit to the MichaelisMenten equation and eq 3.

${ }^{d} K_{d(\text { app })}{ }^{L \text {-Ser }}$ values were determined by the increase in fluorescence at $540 \mathrm{~nm}(\lambda \mathrm{ex}=460$ $\mathrm{nm}$ ), due to the formation of AA, resulting from a titration of $1.0 \mu \mathrm{M}$ ytCBS enzyme with aliquots of L-Ser [Aitken and Kirsch, 2004]. Data were fit to eq 5 . 
Figure 15. Dependence of the observed first-order rate constant $\left(k_{o b s}\right)$ for aminoacrylate formation, monitored at $460 \mathrm{~nm}$, on [L-Ser] for the S289A mutant. The S289A $(16 \mu \mathrm{M})$ mutant was incubated with 5-150 mM L-Ser in $50 \mathrm{mM}$ Tris, $\mathrm{pH} 8.6$ at $25^{\circ} \mathrm{C}$. The line represents the fit of $k_{o b s} v s$. [L-Ser] fit to eq 6. Inset A: Spectra of $16 \mu \mathrm{M} \mathrm{S} 289 \mathrm{~A}$ after 200 $\mathrm{s}$ reaction with 0 (solid line), 15, 50,75, and $150 \mathrm{mM}$ L-Ser (dotted lines) show increasing intensity at 320 and $460 \mathrm{~nm}$, corresponding to the aminoacrylate intermediate, with increasing [L-Ser]. Inset B: Stability of the aminoacrylate intermediate of S289A. The S289A mutant $(16 \mu \mathrm{M})$ was equilibrated in $50 \mathrm{mM}$ Tris, $\mathrm{pH} 8.6$, for 15 min prior to addition of an equimolar concentration of L-Ser and the $460-\mathrm{nm}$ absorbance, corresponding to the aminoacrylate intermediate, was monitored over $360 \mathrm{~min}$ 


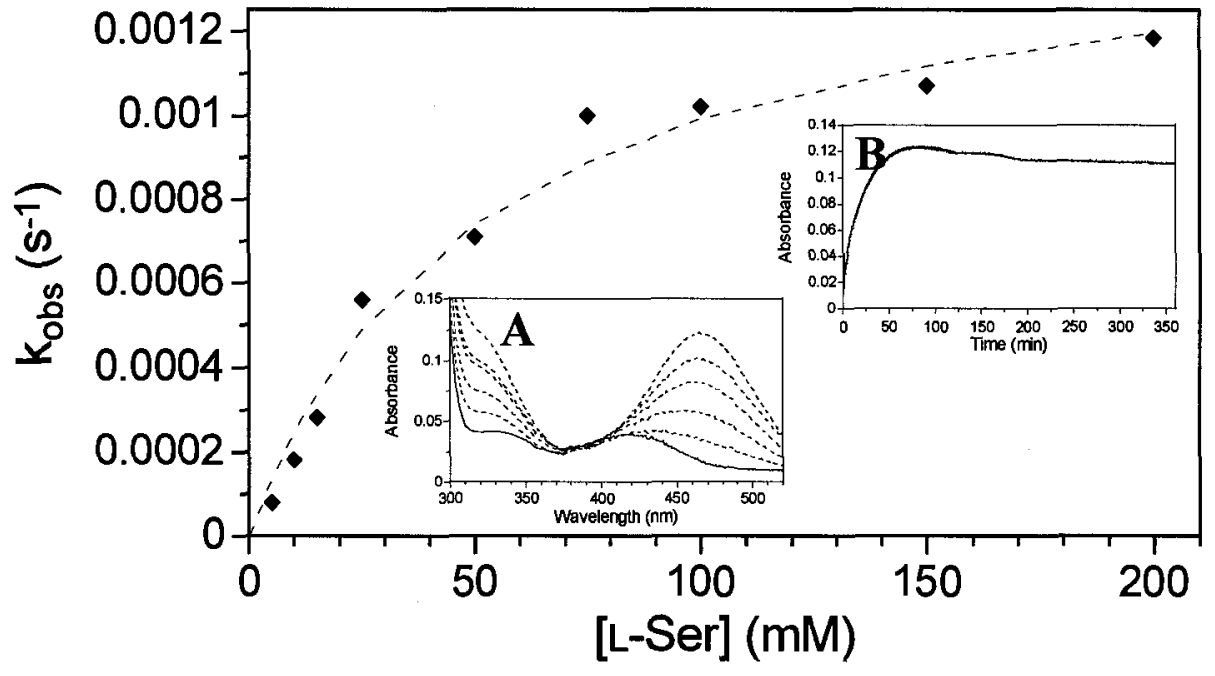


Figure 16. Fluorescence spectra $\left(\lambda_{\mathrm{ex}}=298 \mathrm{~nm}\right)$ of S289A and (Inset) wild-type ytCBS alone (solid line) and in the presence of $50 \mathrm{mM} \mathrm{L}-\mathrm{Ser}$ (dashed line). Conditions: ytCBS or $\mathrm{S} 289 \mathrm{~A}(20 \mu \mathrm{M})$ in $50 \mathrm{mM}$ Tris, $\mathrm{pH}$ 8.6. 


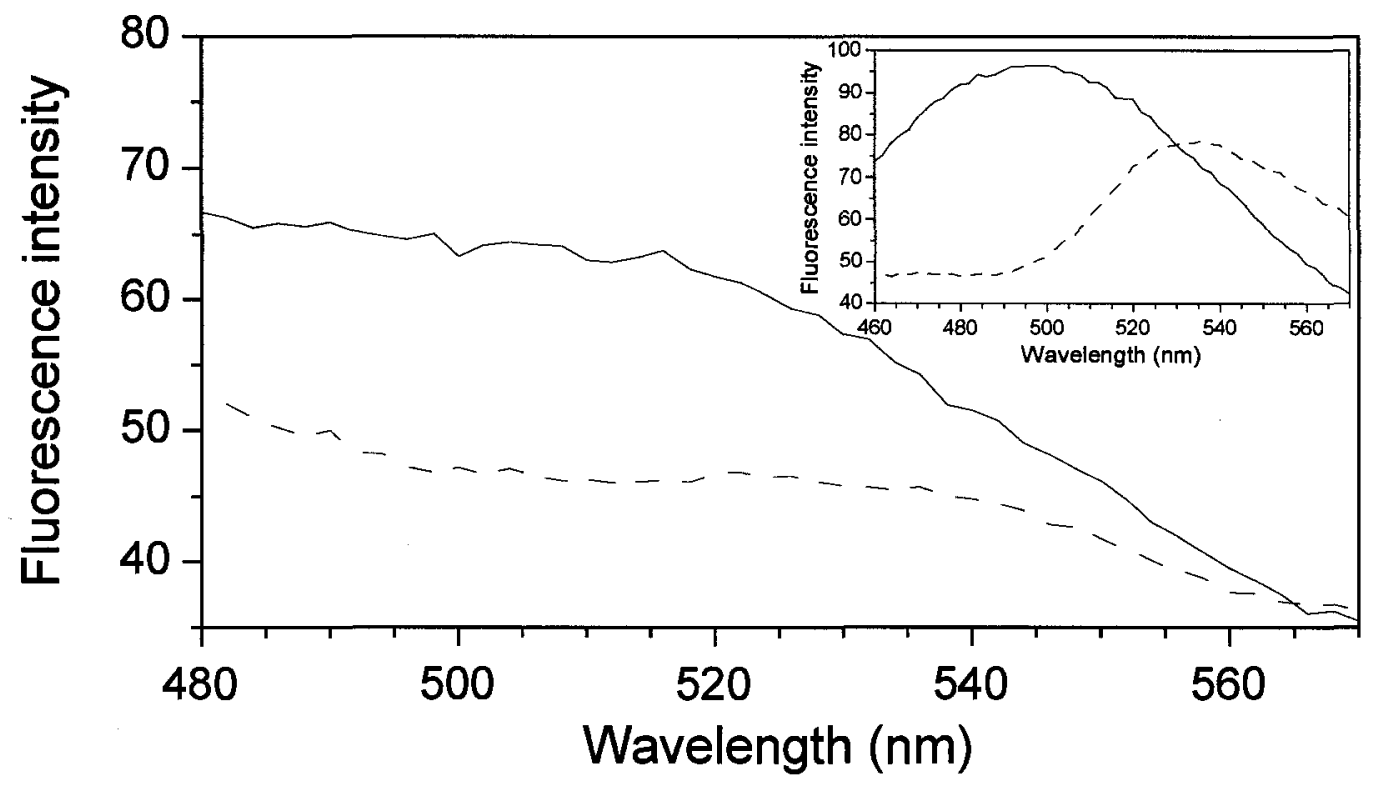




\subsection{Discussion.}

A quinonoid intermediate is observed in $\alpha, \beta$-elimination reactions when elimination of the leaving group is slow, as exemplified by TrpS [Drewe and Dunn, 1985; Daum $e t$ al., 2003]. In contrast, the elimination of acetate from $O$-acetylserine by OASS proceeds via a concerted, $\mathrm{E}_{2}$ mechanism that does not involve a quinonoid [Tai and Cook, 2001; Woehl et al., 1996]. Although the $\alpha, \beta$-elimination of hydroxide from L-Ser, which comprises its first half reaction of CBS, is identical to that of $\operatorname{TrpS}$ and contrasts with the stable acetate leaving group of OASS, the transient quinonoid of TrpS has not been detected for ytCBS, suggesting that either this intermediate of ytCBS does not accumulate or that the enzyme follows an $\mathrm{E}_{2}$ mechanism [Jhee et al., 2001]. Formation of a transient quinonoid in the $E_{1}$ mechanism of TrpS relies upon the presence a hydrogen bond between the hydroxyl group of the side chain of S377 and N1 of the PLP cofactor. Stabilization of this intermediate in Trp-S377D, enabling its detection under equilibrium conditions, coupled with the observed mechanism-based inactivation of this mutant, demonstrates the role of S377 in controlling cofactor chemistry in TrpS [Jhee et al., 1998a; Jhee et al.,1998b]. The presence of a serine residue within hydrogen-bonding distance of N1 of the PLP cofactor is a common feature of TrpS (S377), CBS (S349 of hCBS corresponds to S289 of yCBS) and OASS (S272). However, S377 of TrpS is situated at the C-terminus of a $\beta$-strand and is structurally distinct from S349 of hCBS and S272 of OASS, which are both located at the N-terminus of an $\alpha$-helix [Hyde et al., 1988; Burkhard et al., 1998; Taoka et al., 2002]. Therefore, as ytCBS shares distinct features of TrpS and OASS, the S289A and S289D mutants were constructed to probe the mechanism of this enzyme. 
The S289D mutant of ytCBS lacks detectable $\beta$-replacement, $\beta$-elimination or LCth hydrolysis activity. This contrasts with the weak residual activity of the corresponding TrpS-S377D site-directed mutant. As the PLP saturation of S289D is approximately half that of the wild-type enzyme, the inactivity of this mutant is likely due to improper orientation of the cofactor within the active site. The wild-type enzyme, and to a lesser degree the S289A mutant, exhibit fluorescence emission at $\sim 500 \mathrm{~nm}$, which shifts to $\sim 540 \mathrm{~nm}$ in the presence of L-Ser, due to Forester energy transfer from tryptophan residue(s) of the protein to the PLP cofactor (Figure 16). Similar Forester energy transfer, from W162 to the cofactor, has been observed in OASS [McClure and Cook, 1994; Strambini et al., 1996]. The lack of 500-nm fluorescence, in combination with the $\mathrm{pH}$ independence of the internal aldimine of the S289D mutant, in contrast with the $\mathrm{pH}$ dependence $(\mathrm{pKa}=\sim 7.7)$ of the imine nitrogen of the TrpS-S377D internal aldimine [Jhee et al., 1998a], provide evidence that the orientation of the cofactor in the ytCBS-S289D active site is nonproductive. Subtle changes in the orientation of the PLP cofactor within the active site, compared to the wild-type enzyme, were also observed for the S289A,D mutants of OASS, which retain activity similar to the wild-type, and a key role for S377 in maintaining cofactor orientation has been proposed in TrpS [Cook, 2003; Jhee et al., 1998b].

The S289A mutant of ytCBS reacts with L-Ser to form a stable aminoacrylate intermediate (Figure 15). Therefore, unlike the corresponding S272A mutant of OASS, the rate of decay of this intermediate cannot be employed to determine the $\mathrm{p} K_{a}$ of the active-site lysine (K53) of ytCBS. Presteady-state analysis of aminoacrylate formation by S289A demonstrates that the equilibrium constant, $K_{21}$, for formation of the E^L-Ser 
enzyme-substrate complex (eq 7) is increased by only $\sim 5$-fold. Correspondingly, there is less than $10 \%$ difference in the apparent binding constant of $\left.\mathrm{L}-\mathrm{Ser}\left(K_{d(a p p)}\right)^{L-S e r}\right)$ between the wild-type enzyme and S289A mutant (Table 4). In contrast, the rate of conversion ( $k_{3}$ $\left.=(1.3 \pm 0.1) \times 10^{-3} \mathrm{~s}^{-1}\right)$ of E-L-Ser to the aminoacrylate intermediate (E-AA; eq 7) is reduced by five orders of magnitude, compared to the wild-type enzyme $\left(k_{3}=177 \pm 8 \mathrm{~s}^{-1}\right)$ [Jhee et al., 2001]. The hyperbolic shape of the plot of $k_{o b s}$ for AA formation versus L-Ser concentration indicates that binding of L-Ser is rapid and conversion of the ytCBS•L-Ser complex to the enzyme-bound AA intermediate is the slow step in the first half reaction of both the S289A mutant (Figure 15) and wild-type ytCBS [Jhee et al., 2001]. The S289A mutant of ytCBS catalyzes the $\beta$-replacement and L-Cth hydrolysis activities observed for the wild-type enzyme, as well as a minor $\beta$-elimination of L-Ser. However, the catalytic efficiency of this mutant is strongly reduced, as demonstrated by the $\sim 800$ and $\sim 1400$-fold decreases in $k_{\text {cat }} / K_{m F}^{L-S e r}$ and $k_{c a t R} / K_{m R}{ }^{L-C t h}$ (Table 4) [Aitken and Kirsch, 2004]. A similar effect is observed for TrpS, as the specific activity of the TrpS-S377A,D mutants is decreased to $>100$-fold, compared to the wild-type enzyme, demonstrating that removal of the hydroxyl side chain of S377 drastically reduces the catalytic capacity of this enzyme [Jhee et al., 1998a; Jhee et al., 1997]. In contrast, while the $k_{c a t}$ of the S272A mutant of OASS is reduced by 80 -fold, similar to the 20 -fold decrease in the $k_{\text {cat }}$ of the corresponding S289A mutant of ytCBS, the catalytic efficiency of OASS-S272A is reduced by only 3 -fold because of the compensating 25 -fold decrease in the $K_{m}{ }^{O A S}$ of this mutant [Daum et al., 2003]. The three-order-of-magnitude reduction in the catalytic efficiency of the S289A mutant provides indirect evidence that the ytCBS-catalyzed $\alpha, \beta$ elimination of hydroxide from L-Ser, like that of TrpS, follows an $E_{1}$ mechanism. 
11. Nucleotide recognition by the C-terminal regulatory domain of human Cystathionine $\beta$-Synthase. 


\subsection{Abstract.}

Human Cystathionine $\beta$-synthase is a Pyridoxal 5'-phosphate dependent protein that catalyses the condensation of L-serine and L-homocysteine to L-cystathionine. The enzyme is allosterically regulated and activated $\sim 2$-fold by $S$-adenosylmethionine (SAM) that binds to the $\mathrm{C}$-terminal regulatory domain. The binding site for SAM is unknown due to the lack of a complete crystal structure of CBS. To identify interactions of SAM binding in yeast and human CBS, sequence alignments, disease causing mutations and structural comparison of similar nucleotide-binding motifs from other proteins, were used to target residues for site-directed mutagenesis. Yeast CBS is not activated by the binding of SAM or ATP and the D399A, T414A, S416A, E417A, T498A, and D501A mutants of this enzyme do not display reduced stability or altered kinetic parameters. The $k_{\mathrm{cat}}^{\mathrm{L}-\mathrm{Ser}}$ of the F443A, D444N and S466L mutants of human CBS were increased by 1.5-, 1.7-, and 1.8-fold, while that of D538A was unchanged. The specific activity with $200 \mu \mathrm{M}$ SAM increases by $1.6-$ to 2.2 -fold in the wild type and S466L while it remains unchanged in F443A and D538A. These results provide a basis for future work to establish the mechanism of SAM binding and allosteric activation of human CBS. 


\subsection{Introduction.}

Elevated plasma levels of L-homocysteine (L-Hcys), a toxic metabolite, are an independent risk factor for cardiovascular disease [Mudd et al., 2001]. L-Hcys homeostasis is regulated by the flux of two key enzymes, Cystathionine $\beta$-synthase (CBS), in the transsulfuration pathway, and methylene tetrahydrofolatereductase (MTHFR), in the remethylation pathway [Hajjar, 2001; Figure 6]. MTHFR is inhibited by SAM while CBS binds $S$-adenosylmethionine (SAM), an allosteric activator that causes a $\sim 2-3$ fold increase in the $V_{\max }$ of the enzyme [Taoka et al., 1998; Mato et al., 1997]. The increase in $S$-adenosylmethionine (SAM) concentration, occurring concurrently with elevated L-Hcys levels, results in decreased L-Hcys remethylation, via inhibition of MTHFR to methionine, and an increase in production L-Cystathionine in the transsulfuration pathway [Miles and Kraus, 2004; Banerjee and Zhou, 2005].

Each monomer of the homotetrameric human CBS (hCBS) enzyme is comprised of 551 residues and has a modular domain organization consisting of a N-terminal heme domain ( $\sim 70$ residues), a central catalytic core ( $\sim 340$ residues), and a C-terminal regulatory domain ( $\sim 140$ residues) [Kraus et al., 1978; Miles et al., 2000; Kraus et al., 2001]. The C-terminal regulatory domain is proposed to contain the binding site of the allosteric effector SAM, the binding of which results in a 2-fold increase in the $k_{\mathrm{cat}}$ of the enzyme [Taoka et al., 1998]. Removal of the regulatory domain, either via limited proteolysis or expression of a truncated version of the CBS, results in a SAM-insensitive, homodimeric form of the enzyme, the activity of which is two-fold greater than the wildtype [Taoka et al., 1998; Kery et al., 1998]. Yeast CBS (yCBS) is not activated by SAM but truncation of the C-terminal domain results in activation of the enzyme [Jhee et al., 
2000b]. A subset of the more than 100 homocystinuria-associated mutations of hCBS also alters the responsiveness of the enzyme to SAM. For example, the $\mathrm{K}_{\text {act }}$ value, for activation of hCBS by SAM, is increased $\sim 60$-fold (from 7.4 to $460 \mu \mathrm{M}$ ) in the D444N regulatory-domain mutant, the first characterized regulatory domain mutation with impaired SAM recognition [Kluijtmans et al., 1996; Evande et al., 2002]. In contrast, hCBS-C431S is SAM-insensitive, but has an activity similar to that of the SAM-activated wild-type enzyme [Frank et al., 2006]. Although the S466L mutant of hCBS binds SAM with a $\mathrm{K}_{\mathrm{d}}$ of $2.9 \mu \mathrm{M}$, compared to $11.4 \mu \mathrm{M}$ for wild-type hCBS, its activity is not increased by SAM binding [Frank et al., 2006]. Attempts to crystallize the full-length form of hCBS have failed to date, due to the aggregative character of the enzyme, and the only available crystal structures are of the truncated, homodimeric form of the enzyme, lacking the regulatory domain [Meier et al., 2001; Taoka et al., 2002]. Therefore, the location and nature of the SAM binding site, as well as its relationship with the residues associated with homocystinuria-linked mutations, including D444N, C431S and S466L, have not been determined.

The regulatory domain of CBS contains a pair of CBS domains, named after parent CBS enzyme. These $\sim 60$ amino acid domains comprise a $\beta_{1} \alpha_{1} \beta_{2} \beta_{3} \alpha_{2}$ secondarystructure motif, occur as tandem pairs of two-to-four CBS domains and typically bind adenosine-containing ligands such as ATP, AMP, or SAM [Bateman, 1997]. Proteins containing CBS domains include CBS, inosine 5'monophophate dehydrogenase (IMPDH), AMP-activated protein kinase, and the chloride channel ClC-5. Mammalian CBS is unique as its regulatory domain exclusively binds SAM, while others bind AMP or ATP [Ignoul and Eggermount, 2005]. Although the structure of the regulatory domain 
of the CBS enzyme has not been solved, the structures of CBS domains from several other proteins are available. The low sequence identity between the regulatory domain of hCBS and the CBS domains of available structures present difficulties for homology modeling and binding-site prediction (AMPK $\gamma-3+4,30.6 \%$; AMPK $\gamma-1+2,11 \%$; CLC-5, 14\%; IMPDH, 16\%; SNF4-3+4, 37\%; SNF4-1+2, 35\%). Therefore, information including sequence alignments, structural analysis and disease-associated mutations, of hCBS and other proteins containing CBS domains, were combined to assist in selecting residues, as targets for site-directed mutagenesis, with the goal of investigating the regulatory domain and its interaction with the catalytic core of CBS.

Recent structures of ATP bound to CLC-5 [PDB: 2J9L; Meyer et al., 2007] and AMP bound to AMPK [PDB: 2UV4; Day et al., 2007] reveal interactions with the adenine and ribose components of the ligand, features that are shared with SAM [Figure 8]. The adenine ring in CLC- 5 forms a $\pi$-stacking interacting with the side chain of Y617, but is flanked by a network of hydrophobic residues in AMPK (T200, I204, V225 and I312) [Figure 8]. The human and yeast CBS enzymes share a conserved aromatic residue (F443 and F398 of hCBS and yCBS, respectively), which aligns with Y617 of CLC-5, suggesting the possibility of a similar $\pi$-stacking role for this residue in the SAM-binding site of CBS [Figure 9]. A ribose-binding motif (GhxS/TxS/TD, $x$ is any amino acid and $h$ is hydrophobic) has been identified in AMPK [Day et al., 2007]. The serine/threonine residues and the aspartate residue of the motif are observed to form hydrogen bonds with the hydroxyl groups of the ribose ring and the phosphate moiety, respectively, in AMP or AMP bound to AMPK and CLC-5 [Figure 8]. Putative ribose binding motifs are conserved and present in yCBS: CBS1 (T414, S416, and E417) and 
CBS2 (T498 and D501) (Figure 9). These residues of yCBS (T414A, S416A, E417A, T498A and D501A) were targeted for site-directed mutagenesis. Preliminary work didn't reveal altered kinetic parameters or activation in yCBS. Therefore, other residues were not targeted for a parallel study. In hCBS, the ribose-binding motif in CBS2 domain was selected site-directed mutagenesis (D538A). To test for adenine stacking interactions of SAM in hCBS, F443A was also constructed for site-directed mutagenesis. A single peptide (residues 511-531 of the CBS2 domain) was identified, via mass spectrometry, as protected from ${ }^{1} \mathrm{H} /{ }^{2} \mathrm{H}$-exchange by SAM binding [Sen et al., 2005; Figure 9]. The amino acid sequence of this peptide was aligned with the CBS sequences of higher eukaryotes and four conserved glutamine residues were selected for site-directed mutagenesis (Q515A, Q517A, Q526A, and Q528A) to investigate their role in the regulatory domain of hCBS. The disease mutations of homocystinuria located in the CBS domains, C431S, D444N and S466L, were also developed. The soluble enzymes were purified and their kinetic parameters, activation by SAM, and protein stability were compared to the wildtype enzymes. Comparison of the effect of mutations in hCBS with the corresponding mutants of the SAM-insensitive yeast enzyme provide insight into the regulation of CBS. 


\subsection{Methods}

\subsubsection{Construction, Expression, and Purification of yeast and human CBS mutants.}

Overlap-extension PCR, with the pSECseq0 and pSECseq $7 r$ flanking primers and the appropriate pairs of mutagenic primers (Table 5) was employed to introduce the C431S, F443A, D444N, T459A, S466L, Q515A, Q517A, Q526A, Q528A, and D538A site-directed mutations in the 6-His/hCBS construct (described in Section 9.3.3 of this thesis) [Higuchi, 1990]. The amplification product was digested with $N c o$ I and SalI, inserted at the corresponding sites of the $\mathrm{pTrc} 99 \mathrm{a}-6-\mathrm{His} / \mathrm{hCBS}$ vector and transformed into the $E$. coli strain DH10B (Gibco BRL). The D399A, D474A, T414A, S416A, E417A, T498A and D501A of yCBS were constructed using overlap-extension PCR with the $\mathrm{pSECseq0}$ and $\mathrm{pSECseq} 7 \mathrm{r}$ flanking primers and appropriate mutagenic primers from a yCBS-His construct (Table 5), digested with $N d e I$ and SalI, introduced into the corresponding sites of the pTrc99a expression vector and transformed into $E$. coli strain DH10B (Gibco BRL). The yCBS mutants (D399A, D474A, T414A, S416A, E417A, T498A, and D501A) and hCBS mutants (F443A, D444N, T459A, and D538A) were sequenced and verified. The mutant enzymes were expressed and purified via Ni-NTA affinity chromatography (Qiagen), as described for the heterologous expression of 6-His tagged hCBS and yCBS in Sections 9.3.5. and 10.3.2. 
Table 5. Primer sequences used for constructing C-terminal domain mutants in yCBS and hCBS.

\begin{tabular}{|c|c|c|c|c|c|c|c|c|c|c|}
\hline Oligo name & & & 歌 & Seq & Henc & $\mathrm{ces}^{3}$ & $=3$ & Why & W & W \\
\hline pSECseq0 & GGC & GTC & AGG & CAG & CCA & TCG & GAA & GCT & G & \\
\hline pSECseq $7 r$ & GCC & CGC & CAC & CCT & CCG & GGC & CGT & TGC & TTC & $\mathbf{G}$ \\
\hline hCBS C431Sf $f^{b}$ & CGA & CCA & TCA & CCA & GCG & GGC & $\mathrm{ACA}$ & $\mathrm{CCA}$ & TCG & AG \\
\hline $\mathrm{hCBS} \mathrm{C} 31 \mathrm{Sr}^{b}$ & CTC & GAT & GGT & GTG & $\mathrm{CCC}$ & GCT & GGT & GAT & GGT & CG \\
\hline hCBS F443Af & CGG & GAG & AAG & GGC & GCG & GAC & CAG & GCG & & \\
\hline hCBS F443Ar & CGC & CTG & GTC & CGC & GCC & CTT & CTC & CCG & & \\
\hline hCBS D444Nf & AGA & AGG & GCT & TCA & $\mathrm{ACC}$ & AGG & CGC & CCG & TG & \\
\hline hCBS D444Nr & CAC & GGG & CGC & CTG & GTT & GAA & GCC & CTT & CT & \\
\hline hCBS T459Af & GAA & TGG & TGG & CGC & TTG & GGA & $\mathrm{ACA}$ & TG & & \\
\hline hCBS T459Ar & CAT & GTT & $\mathrm{CCC}$ & AAG & CGC & CAC & CAT & $\mathrm{TC}$ & & \\
\hline hCBS S466Lf & GAA & CAT & GCT & CTC & GCT & GCT & GCT & TGC & CGG & GAA \\
\hline hCBS S466Lr & TTC & CCG & GCA & AGC & $\mathrm{AGC}$ & AGC & GAG & AGC & ATG & TTC \\
\hline hCBS Q515Af & GGT & GGT & GCA & CGA & GGC & GAT & CCA & GTA & $\mathrm{CCA}$ & $\mathrm{C}$ \\
\hline hCBS Q515Ar & GTG & GTA & CTG & GAT & CGC & CTC & GTG & CAC & CAC & $\mathrm{C}$ \\
\hline hCBS Q517Af & GCA & CGA & GCA & GAT & CGC & GTA & $\mathrm{CCA}$ & CAG & CAC & C \\
\hline hCBS Q517Ar & GGT & GCT & GTG & GTA & CGC & GAT & CTG & CTC & GTG & $\mathrm{C}$ \\
\hline hCBS Q526Af & CGG & GAA & GTC & CAG & TGC & GCG & GCA & GAT & GGT & $\mathbf{G}$ \\
\hline hCBS Q526Ar & CAC & CAT & CTG & CCG & CGC & ACT & GGA & CTT & $\mathrm{CCC}$ & G \\
\hline hCBS Q528Af & GTC & CAG & TCA & GCG & GGC & GAT & GGT & GTT & CGG & GG \\
\hline hCBS Q528Ar & $\mathrm{CCC}$ & CGA & $\mathrm{ACA}$ & $\mathrm{CCA}$ & TCG & $\mathrm{CCC}$ & GCT & GAC & TGG & $A C$ \\
\hline hCBS D538Af & $A C C$ & GCC & ATT & GCG & TTG & CTG & $A A C$ & TTC & G & \\
\hline hCBS D538Ar & CGA & AGT & TCA & GCA & ACG & CAA & TGG & CGG & $\mathbf{T}$ & \\
\hline yCBS-D399Af & GAC & AAT & GGC & TTT & GCG & CAA & TTG & $\mathrm{CCT}$ & G & \\
\hline yCBS-D399Ar & CAG & CGA & ATT & GCG & CAA & AGC & CAT & TGT & C & \\
\hline yCBS-T414Af & TCT & GGT & TTA & GTT & GCG & CTC & TCT & GAG & $\mathrm{C}$ & \\
\hline yCBS-T414Ar & GCT & CAG & $\mathrm{AGA}$ & GCG & CAA & CTA & AAC & CAG & A & \\
\hline yCBS-S416Af & GTT & $\mathrm{ACT}$ & CTC & GCG & GAG & CTT & CTA & AG & & \\
\hline yCBS-S416Ar & CTT & $A G A$ & AGC & TCC & GCG & $\mathrm{AGA}$ & GTA & $A C$ & & \\
\hline yCBS-E417Af & GTT & $\mathrm{ACT}$ & CTC & TCT & GCG & CTT & CTA & $A G$ & & \\
\hline yCBS-E417Ar & CTT & $\mathrm{AGA}$ & AGC & GCA & GAG & $\mathrm{AGA}$ & GTA & $\mathrm{AC}$ & & \\
\hline yCBS-D474Af & CAA & AGC & TAT & CTG & CGT & TGA & ATC & GTT & TC & \\
\hline yCBS-D474Ar & GAA & ACG & ATT & CAA & GCG & AGA & TAG & CTT & TG & \\
\hline yCBS-T498Af & ATC & CAT & ATC & GTT & GCG & AAG & ATG & GAT & $\mathbf{T}$ & \\
\hline yCBS-T498Ar & AAT & $\mathrm{CCA}$ & TCT & TCG & CAA & CGA & TAT & GGA & $\mathbf{T}$ & \\
\hline yCBS-D501Af & GTT & $\mathrm{ACT}$ & AAG & ATG & GCG & TTA & CTG & AGC & & \\
\hline yCBS-D501Ar & GCT & CAG & TAA & CGC & CAT & CTT & AGT & AAC & & \\
\hline
\end{tabular}

${ }^{a}$ Primer names starting with $\mathrm{h}$ and $\mathrm{y}$ were employed in the construction of site-directed mutants of human and yeast CBS, respectively.

${ }^{b} \mathrm{C} 431 \mathrm{~S}$ not constructed. 


\subsubsection{SAM/ATP activation of yeast and human CBS.}

Measurements of CBS activation by SAM or ATP were conducted in assay buffer comprising $50 \mathrm{mM}$ Tris, $\mathrm{pH} 8.6,0.5 \mathrm{mg} / \mathrm{ml}$ BSA and $20 \mu \mathrm{M}$ PLP. The enzyme was incubated with $1000 \mu \mathrm{M}$ SAM for two hours on ice minutes prior to assaying activity. A background rate for the coupled-coupled CBL-LDH assay was obtained with $1.5 \mathrm{mM}$ $\mathrm{NADH}, 1.6 \mu \mathrm{M}$ CBL, $1.4 \mu \mathrm{M}$ LDH, $2 \mathrm{mM}$ L-Hcys and $30 \mathrm{mM}$ L-Ser and the reaction was initiated by the addition of $0.8-1.5 \mu \mathrm{M}$ hCBS, preincubated with SAM. The final concentration of SAM in the assay was $200 \mu \mathrm{M}$. A similar specific activity assay was also done with 0-5M ATP for yCBS and hCBS.

\subsubsection{Urea denaturation - yeast CBS stability.}

The stability of the wild-type and site-directed mutants of CBS was compared using fluorescence-monitored urea denaturation with a Cary Eclipse (Varian) spectrofluorimeter. Preliminary studies indicated that a 24-hr incubation of 10-20 $\mu \mathrm{M}$ yCBS in 0-8M urea was sufficient to reach equilibrium between folded and unfolded protein states. All samples were incubated in $10 \mathrm{mM}$ Tris, $\mathrm{pH} 8.6$ for $24 \mathrm{hrs}$ at $4{ }^{\circ} \mathrm{C}$ and measurements were taken at $25^{\circ} \mathrm{C}\left(\lambda_{\mathrm{ex}}=280 \mathrm{~nm}, \lambda_{\mathrm{em}}=300-400 \mathrm{~nm}\right)$. Since the yield of hCBS, wild type and mutants, is low, the stability data was collected in greater detail for the yeast enzymes. Only measurements for wild type hCBS were limited to urea concentrations of $0 \mathrm{M}, 1 \mathrm{M}, 2 \mathrm{M}, 3 \mathrm{M}, 4 \mathrm{M}, 5 \mathrm{M}, 6 \mathrm{M}$ and $7 \mathrm{M}$ (not shown) while the sitedirected mutants were prioritized for kinetics and preliminary SAM-binding studies. 
At each urea concentration, the fraction of unfolded protein, $\mathrm{f}$, was calculated using:

$$
f=I_{o b s}-I_{U} / I_{N}-I_{U}
$$

where $I_{o b s}$ is the observed fluorescence intensity, $I_{U}$ is the fluorescence intensity of the unfolded protein state, and $I_{N}$ is the fluorescence intensity of the native protein state [Liu et al., 2001]

\subsubsection{Steady-state kinetics of yeast and human CBS domain mutants.}

Kinetic measurements were done out as described previously (Section 9, Table 2). Assay conditions were $50 \mathrm{mM}$ Tris (pH 8.6) containing $20 \mu \mathrm{M}$ PLP, $0.5 \mu \mathrm{M}$ CBL, 1.4 $\mu \mathrm{M}$ LDH, $1.5 \mathrm{mM} \mathrm{NADH}$, and $0.63-1.7 \mu \mathrm{M}$ yCBS at $25^{\circ} \mathrm{C} . \beta$-replacement activity was measured by varying L-Hcys concentrations, $0.1-7 \mathrm{mM}$, at $20 \mathrm{mM} \mathrm{L-Ser}$ and by varying L-Ser concentrations, $0.2-35 \mathrm{mM}$, at $2 \mathrm{mM}$ L-Hcys. For hCBS wild type and its sitedirected mutants, similar assay conditions were used (except for 1.6 $\mu \mathrm{M}$ CBL). $0.4-1.2$ $\mu \mathrm{M}$ hCBS $\beta$-replacement activity was measured by varying L-Hcys concentrations, 0.1 $9 \mathrm{mM}$, at $30 \mathrm{mM} \mathrm{L-Ser}$ and by varying L-Ser concentrations, $0.2-50 \mathrm{mM}$, at $2 \mathrm{mM} \mathrm{L-}$ Hcys. $k_{c a t}$ and $K_{m}$ values were obtained from the Kaleidagraph software by fitting the data to the Michaelis-Menten equation, while $k_{c a t} / K_{m}$ was determined independently from eq 10.

$$
v / E=k_{c a t} \frac{k_{c a t} / K_{m}[S]}{1+[S] / K_{m}}
$$




\subsection{Results}

\subsubsection{Purification and characterization of Wt and mutant yeast $C B S$.}

The yeast model enzyme was initially selected for investigating the effect of mutations on the CBS domains because the expression system for hCBS was under development and optimization.

Seven site-directed mutants of yCBS were constructed (D399A, T414A, S416A, E417A, D474A, T498A and D501A), expressed, and purified with yields ranging from 18 - $46 \mathrm{mg} / \mathrm{L}$ of media (Figure 17; Table 2). Only the D474A mutant did not fold properly and was found in inclusion bodies of the cell pellet, as verified by running NiNTA chromatography, under denaturing conditions, and SDS-PAGE (gel picture not shown). The urea denaturation curves show that the introduced mutations do not affect the conformational stability of yCBS but E417A appears to be more stable than the wild type and other mutants [Figure 18]. At substrate concentrations of $5 \mathrm{mM} \mathrm{L-Ser}$ and $1 \mathrm{mM}$ L-Hcys, ligand concentrations of 0-5 mM SAM, ATP, and ATP + methionine had no effect on yCBS as the specific activity of the wild-type enzyme in the presence of $0-5$ $\mathrm{mM}$ of adenine ligands was between $126 \pm 11 \mathrm{U} / \mathrm{mg}$ and $118 \pm 23 \mathrm{U} / \mathrm{mg}$ for SAM, $130 \pm$ $10 \mathrm{U} / \mathrm{mg}$ and $145 \pm 13 \mathrm{U} / \mathrm{mg}$ for ATP, and $134 \pm 12 \mathrm{U} / \mathrm{mg}$ and $145 \pm 11 \mathrm{U} / \mathrm{mg}$ for ATP + Met. Since, the wild-type yeast enzyme was not activated by SAM, the kinetic experiments on yCBS mutants were not conducted with SAM. The $K_{\mathrm{m}}{ }^{\mathrm{L}-\mathrm{Ser}}(\sim 2 \mathrm{mM})$ and $k_{\text {cat }}^{L-S e r}\left(\sim 3 \mathrm{~s}^{-1}\right)$ values of the mutants are not altered, compared to the wild-type enzyme [Table 6, Figure 19]. In contrast, comparison of the kinetic parameters for variation of LHcys at fixed L-Ser concentration reveals 2.7-4.3-fold increases in $K_{\mathrm{m}}{ }^{\mathrm{L}-\mathrm{Hcys}}$ for the E417A, T498A, and D501A mutants. 


\subsubsection{Purification and characterization of Wt and mutant human CBS.}

Seven site-directed mutants of hCBS (F443A, D444N, S466L, Q515A, Q526A, Q528A and D538A) were successfully expressed and purified (Figure 17B). Their yield was between $0.4-3.7 \mathrm{mg} / \mathrm{L}$ of media (Table 7). The T459A and Q517A mutants could not be purified, likely due to improper folding, while the C431S mutant could not be successfully constructed via overlap-extension PCR. A fluorescence assay was used to measure SAM binding. This assay measures changes in the internal protonated aldimine $\left(\lambda_{\mathrm{ex}}-420 \mathrm{~nm}\right)$ environment caused by conformational changes in the PLP microenvironment [Frank et al., 2006]. However, no measurable changes in the fluorescence of the PLP cofactor were detected. Therefore, specific activity assays were used to assess SAM activation.

The steady-state kinetic parameters of the wild type and the seven site-directed mutants of hCBS (F443A, D444N, S466L, Q515A, Q526A, Q528A and D538A) were

determined (Table 7). With the exception of F443A $\left(K_{m}{ }^{\mathrm{L}-\mathrm{Ser}}=6.1 \pm 0.4 \mathrm{mM}\right)$, the $K_{m}{ }^{\mathrm{L}-\mathrm{Ser}}$ values are within experimental error of the wild-type enzyme $\left(K_{m}{ }^{\mathrm{L}-\mathrm{Ser}}=4.2 \pm 0.5 \mathrm{mM}\right)$ (Figure 20). Similarly, the $K_{m}{ }^{\mathrm{L}-\mathrm{Hcys}}$ values of the site-directed mutants are within experimental error of the wild type $(0.32 \pm 0.07 \mathrm{mM})$ (Table 7). The Q526A and Q528A mutants, however, did not display detectable activity.

The specific activity of D538A is comparable to the wild type $(221 \pm 7 \mathrm{U} / \mathrm{mg})$ but is 1.9-fold in lower in Q515A (114 $\pm 10 \mathrm{U} / \mathrm{mg})$. The F443A, D444N, and S466L display 2.2-, 2.2-, and 2.4-fold increase in specific activities compared to the wild type. Frank $e$ t al. showed that $200 \mu \mathrm{M}$ SAM is sufficient to effect a maximal activation [2008]. In the presence of $200 \mu \mathrm{M}$ SAM, the specific activities of D538A (205 $\pm 29 \mathrm{U} / \mathrm{mg})$ and Q515A 
$(111 \pm 7 \mathrm{U} / \mathrm{mg}$ ) remain similar. D444N, which was twice as active, does not change with SAM addition ( $455 \pm 33 \mathrm{U} / \mathrm{mg})$. SAM, however, induces a 1.6-fold increase in the specific activities of the wild type $(345 \pm 42 \mathrm{U} / \mathrm{mg})$ and the $\mathrm{S} 466 \mathrm{~L}(817 \pm 81 \mathrm{U} / \mathrm{mg})$ mutant, respectively.

Interestingly, at $5 \mathrm{mM} \mathrm{L-Ser}$ and $1 \mathrm{mM} \mathrm{L-Hcys}$ substrate concentrations, preliminary experiments show that in the presence of $5 \mathrm{mM} \mathrm{ATP}$, the specific activity of hCBS increases by 2.6 -fold from $81 \pm 5 \mathrm{U} / \mathrm{mg}$ to $211 \pm 6 \mathrm{U} / \mathrm{mg}$. However, at $0-3 \mathrm{M}$ ATP, hCBS activity remains similar. 
Figure 17. SDS-PAGE of 6-His-tagged yCBS and hCBS site-directed mutant enzymes. (A) SDS-PAGE of fractions from purification of yCBS-D501A by Ni-NTA chromatography. Lane 6 and 17: protein marker; lane 1: cell lysate supernatant; lane 2: column flow-through following loading; lane 3: column wash, lanes 4, 5, 7-16 and 1820: every fifth protein fraction from the elution, starting with the first fraction. (B) SDSPAGE of fractions from the purification of hCBS-D444N by Ni-NTA chromatography. Lane 5 and 14: protein marker; lane 1: cell lysate supernatant; lane 2: column flowthrough following loading; lane 3: column wash; lanes 4, 5-13 and 15-17: every third fraction from the elution, starting from the first fraction. The $10 \%$ polyacrylamide gel was loaded with protein fractions per lane and stained with Coomassie brilliant blue. 
A.

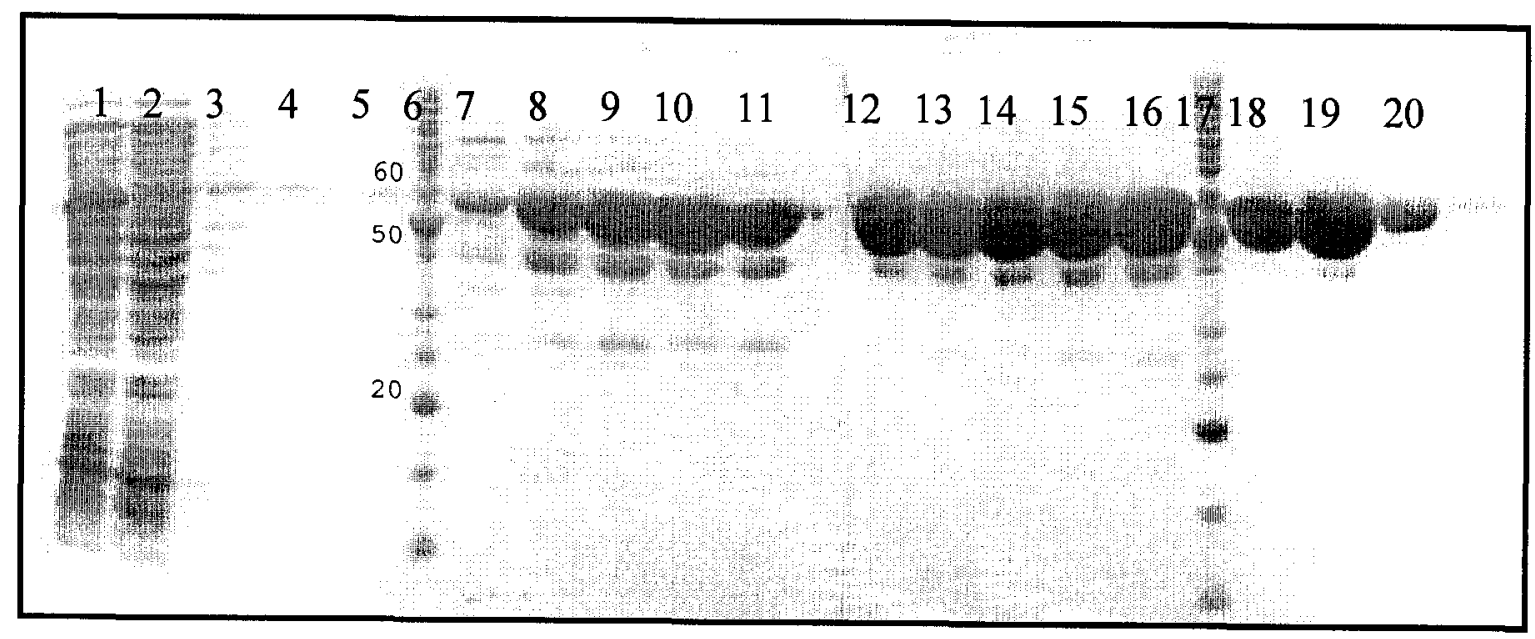

B.

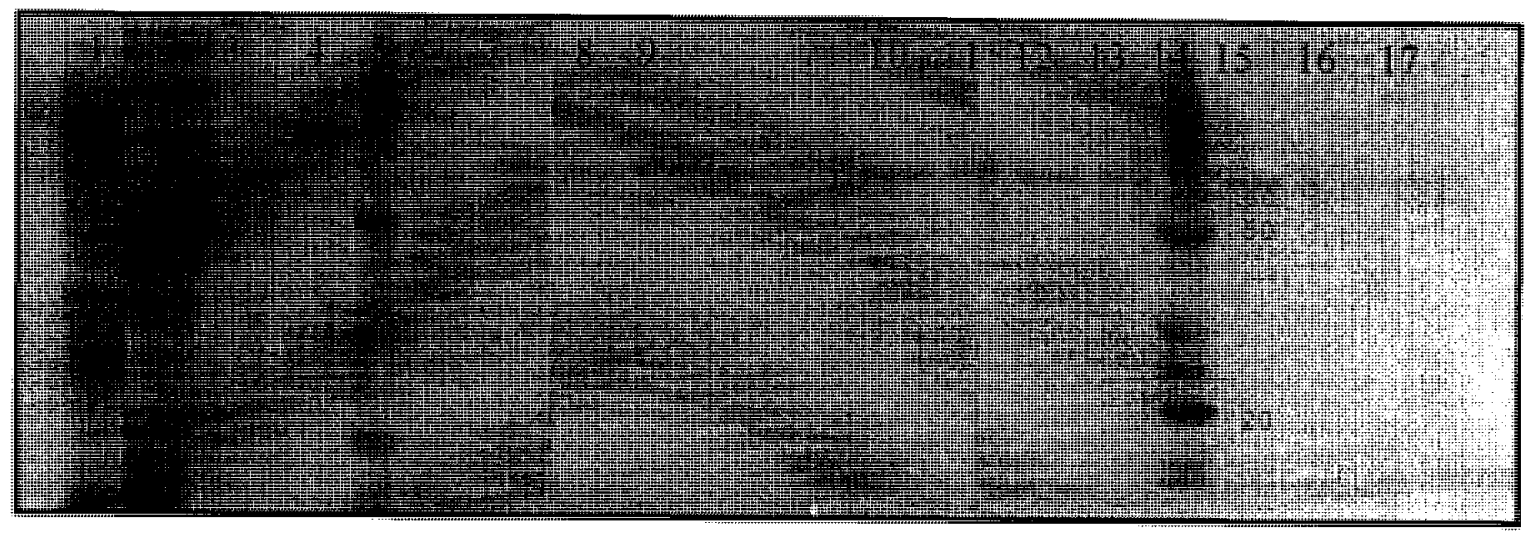


Figure 18. Fluorescence-monitored denaturation of yCBS by 0-8 $\mathrm{M}$ urea. (A) The denaturation curves of yCBS $(\square)$, D399A $(\diamond)$, E417 (×), and D501A (O) yCBS samples were incubated overnight at $4{ }^{\circ} \mathrm{C}$ overnight in $10 \mathrm{mM}$ Tris, $\mathrm{pH} 8.6$ containing 0 $8 \mathrm{M}$ urea. The other mutants, T414A, S416A, and T498A have similar protein unfolding equilibriums as the wild type and to maintain the pictures clarity, the data is not shown. The fluorescence intensities (f) at $330 \mathrm{~nm}\left(\lambda_{\mathrm{ex}}=280 \mathrm{~nm}\right)$ were normalized by equation 9 and plotted versus urea concentration. B. Emission spectra $\left(\lambda_{\mathrm{ex}}=280 \mathrm{~nm}\right)$ of $20 \mu \mathrm{M}$ yCBS-T414A showing the increasing peak at showing the increasing peak at $\sim 350 \mathrm{~nm}$ with increasing urea concentration $(0,2,2.25,2.5,2.75,3,3.25,3.5,4,4.5,5$, and $7 \mathrm{M})$. 
A.

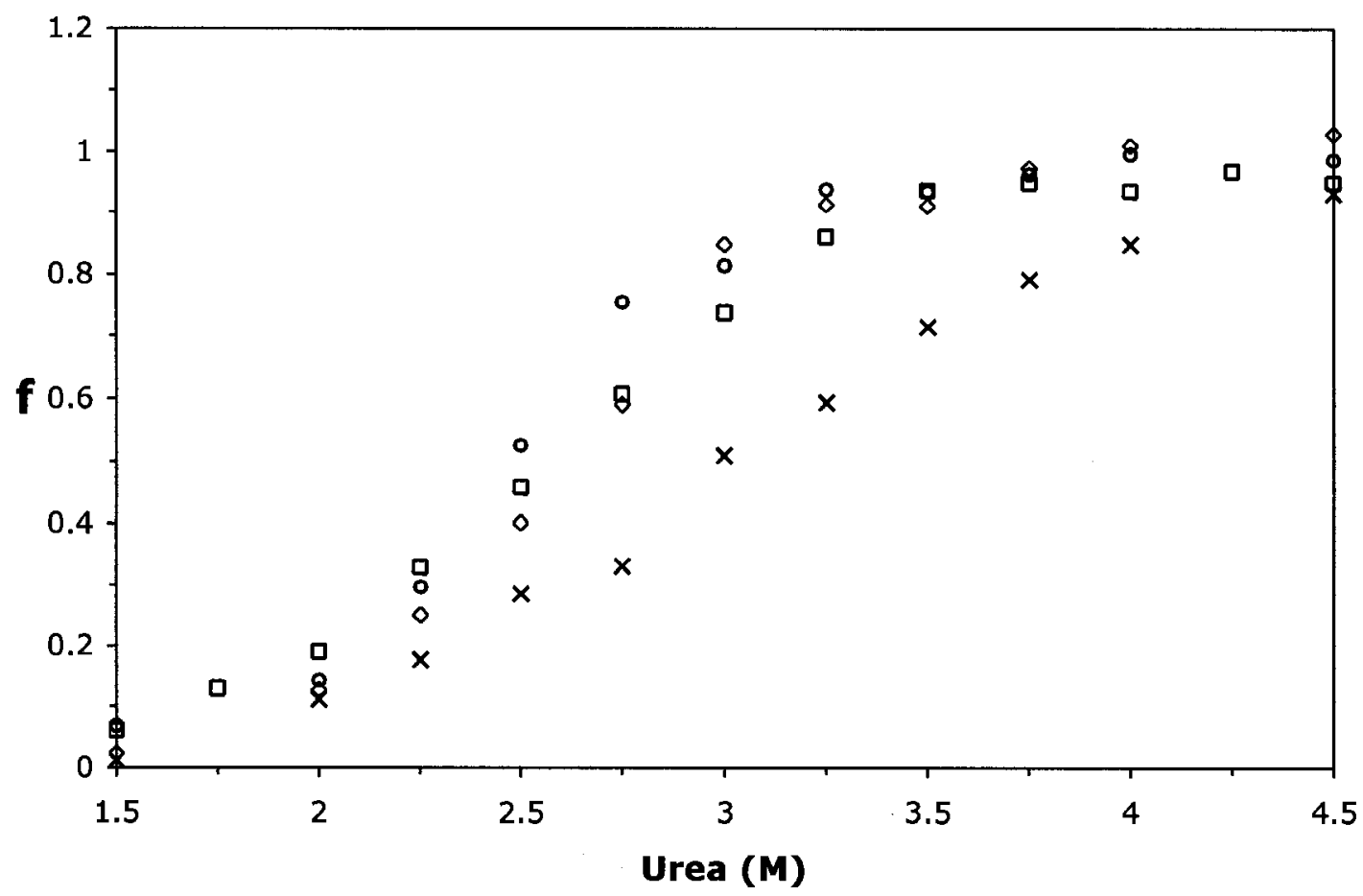

B.

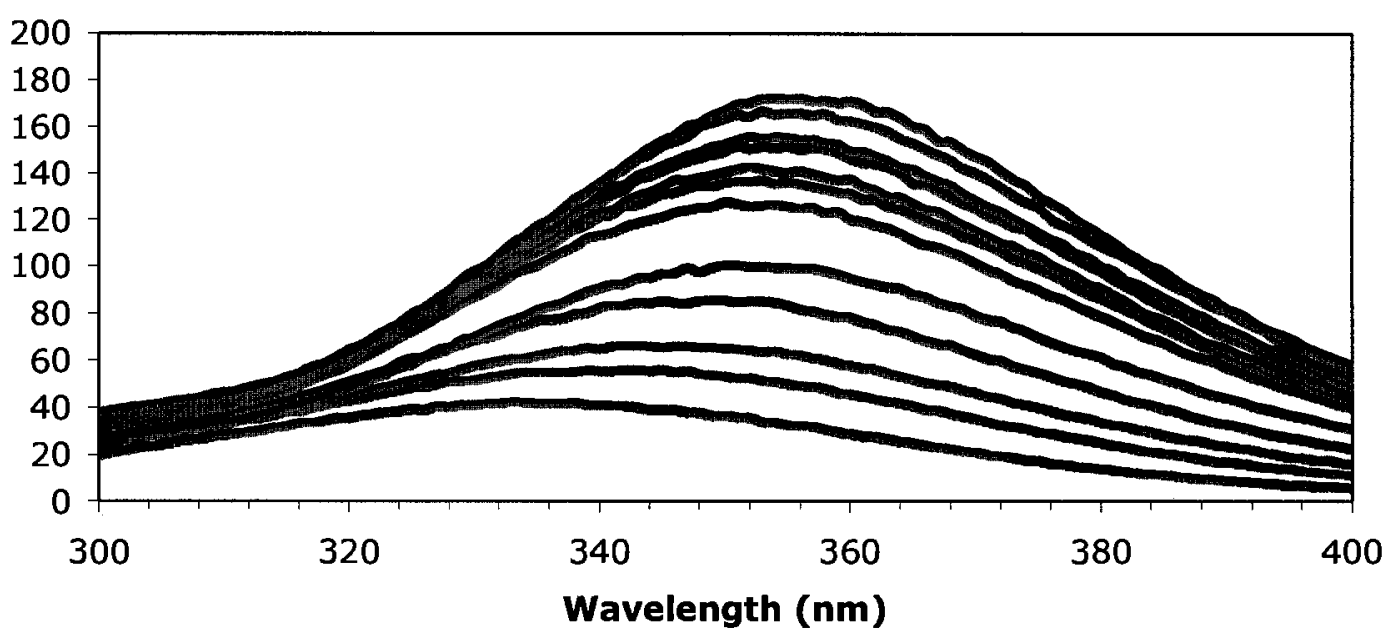


Figure 19. Michaelis-Menten plots demonstrating the dependence of the reaction catalyzed by wild-type yCBS $(\diamond)$ and the D399A $(\square)$, and S416A $(\times)$ site-directed mutants on (A) varying [L-Ser] at a fixed L-Hcys concentration of $2 \mathrm{mM}$ and (B) varying [L-Hcys] at a fixed L-Ser concentration of $20 \mathrm{mM}$. The kinetic parameters are given in Table 6. Reactions conditions were $50 \mathrm{mM}$ Tris (pH 8.6), $1.5 \mathrm{mM} \mathrm{NADH}, 20 \mu \mathrm{M}$ PLP, $0.5 \mu \mathrm{M} \mathrm{CBL}, 1.4 \mu \mathrm{M} \mathrm{LDH}$ and $0.6-1.7 \mu \mathrm{M}$ wild-type or mutant yCBS. 
A.

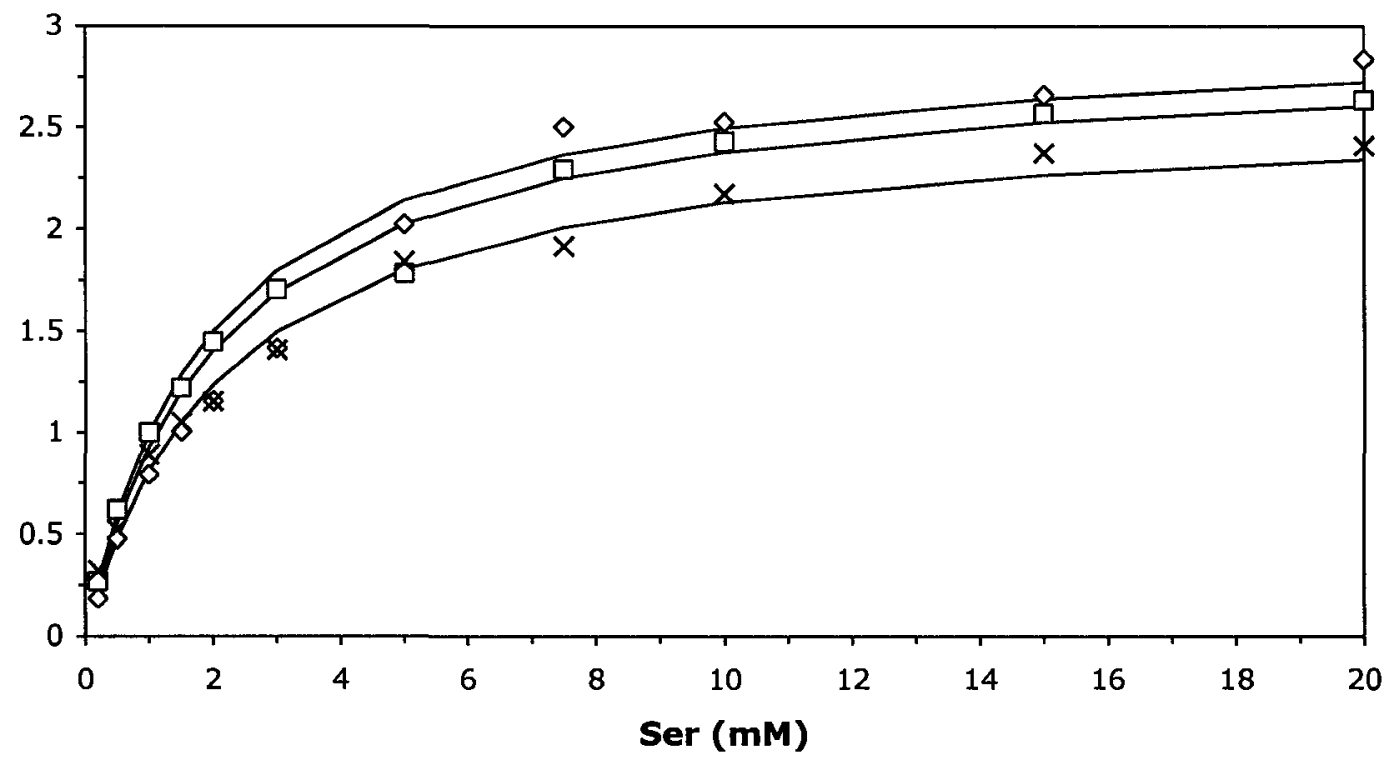

B.

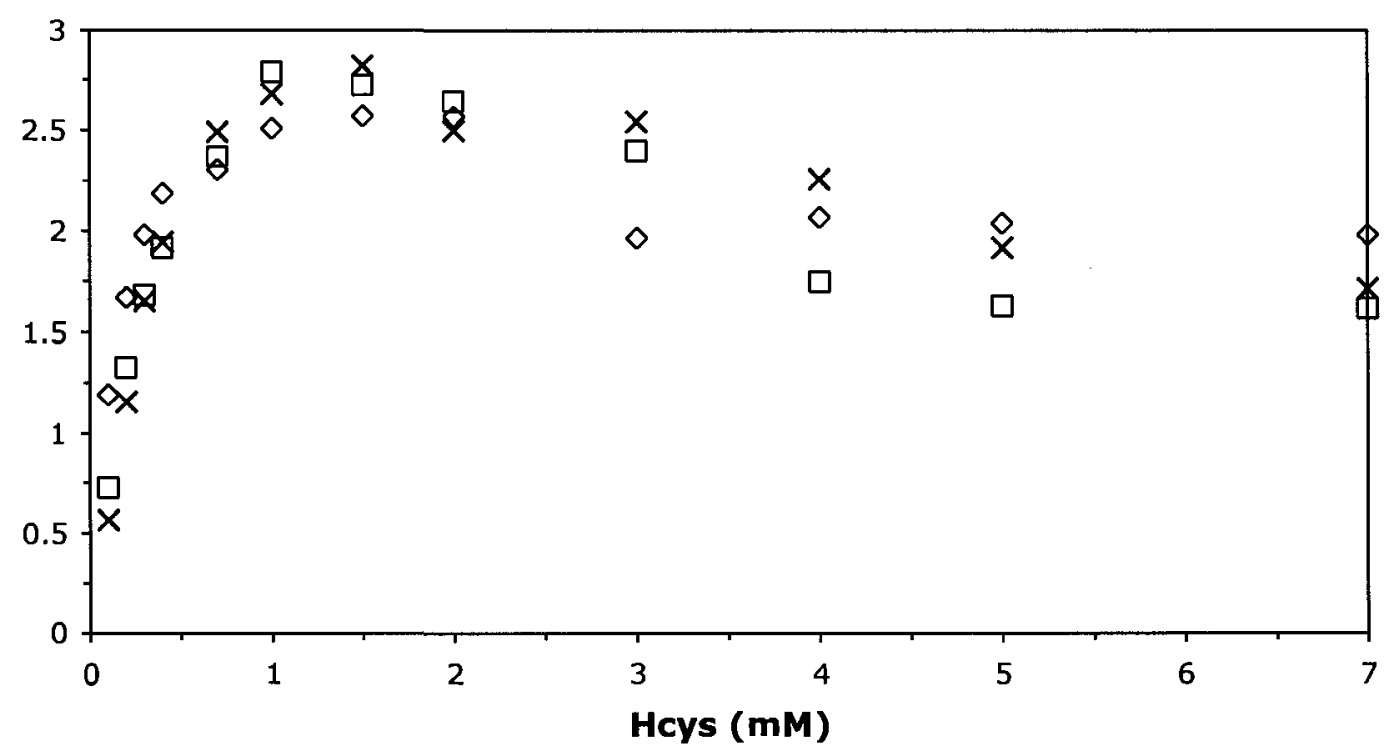


Figure 20. Kinetics of hCBS proteins showing the dependence of the reaction on A. [LSer] with concentration of L-Hcys fixed at $2 \mathrm{mM}$ and B. [L-Hcys] with concentration of LSer fixed at $30 \mathrm{mM}$. ( $\diamond$ - hCBS; $\square-\mathrm{F} 443 \mathrm{~A}$; - D538A). The fitted parameters are given in Table 7. Reactions conditions were $50 \mathrm{mM}$ Tris (pH 8.6), $1.5 \mathrm{mM}$ NADH, $20 \mu \mathrm{M}$ PLP, 1.6 $\mu \mathrm{M} \mathrm{CBL}$, and 1.4 $\mu \mathrm{M} \mathrm{LDH}$. Reactions were initiated by addition of $0.3-1.2$ $\mu \mathrm{M}$ hCBS. 
A.

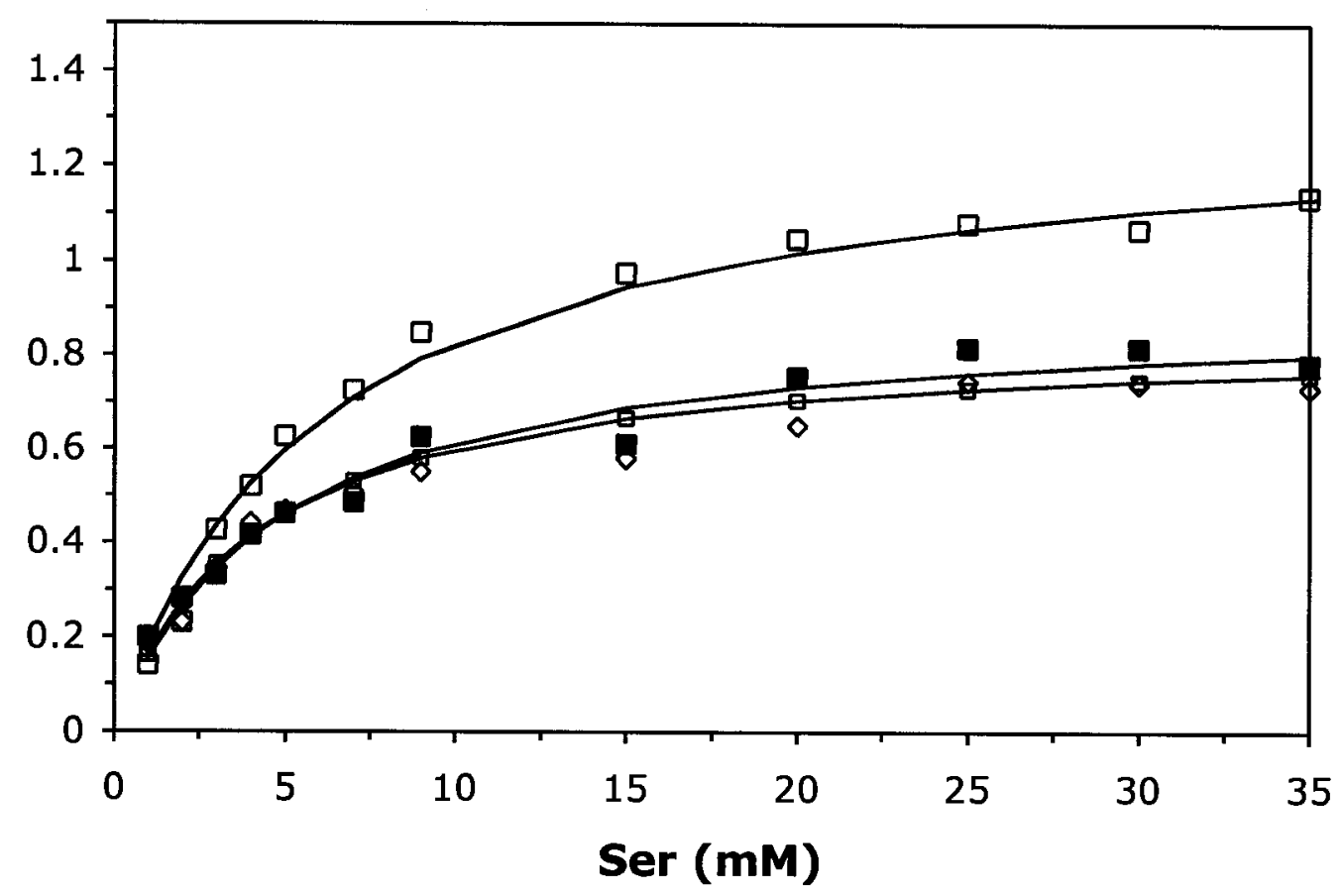

B.

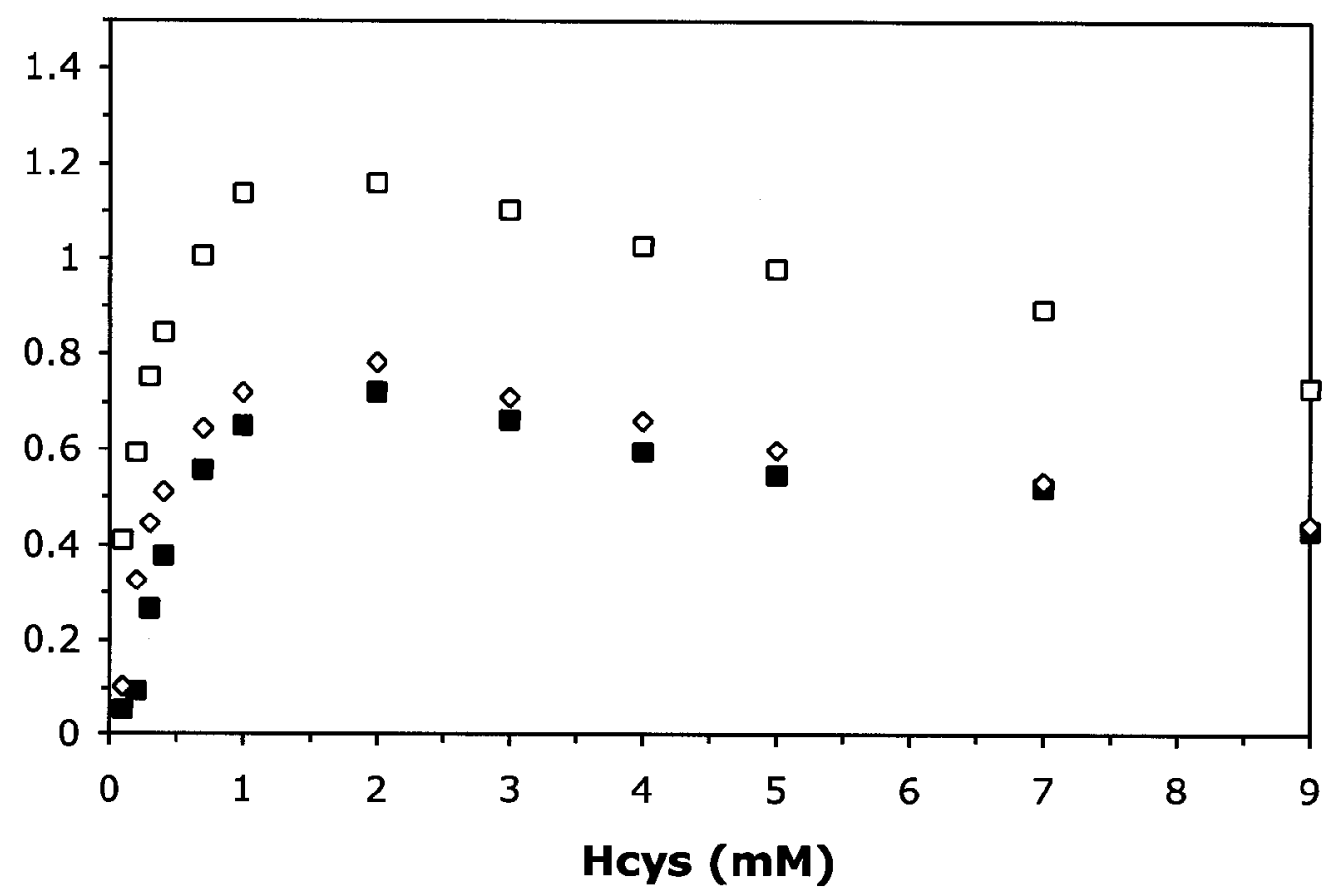




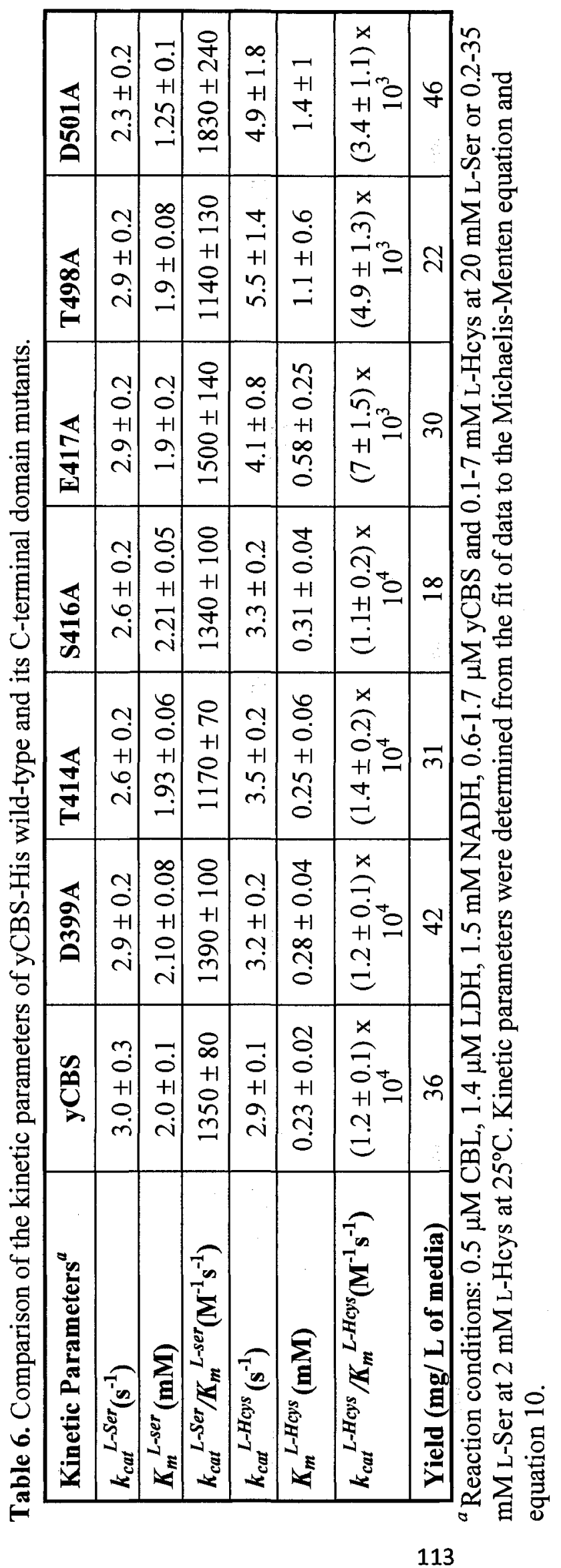




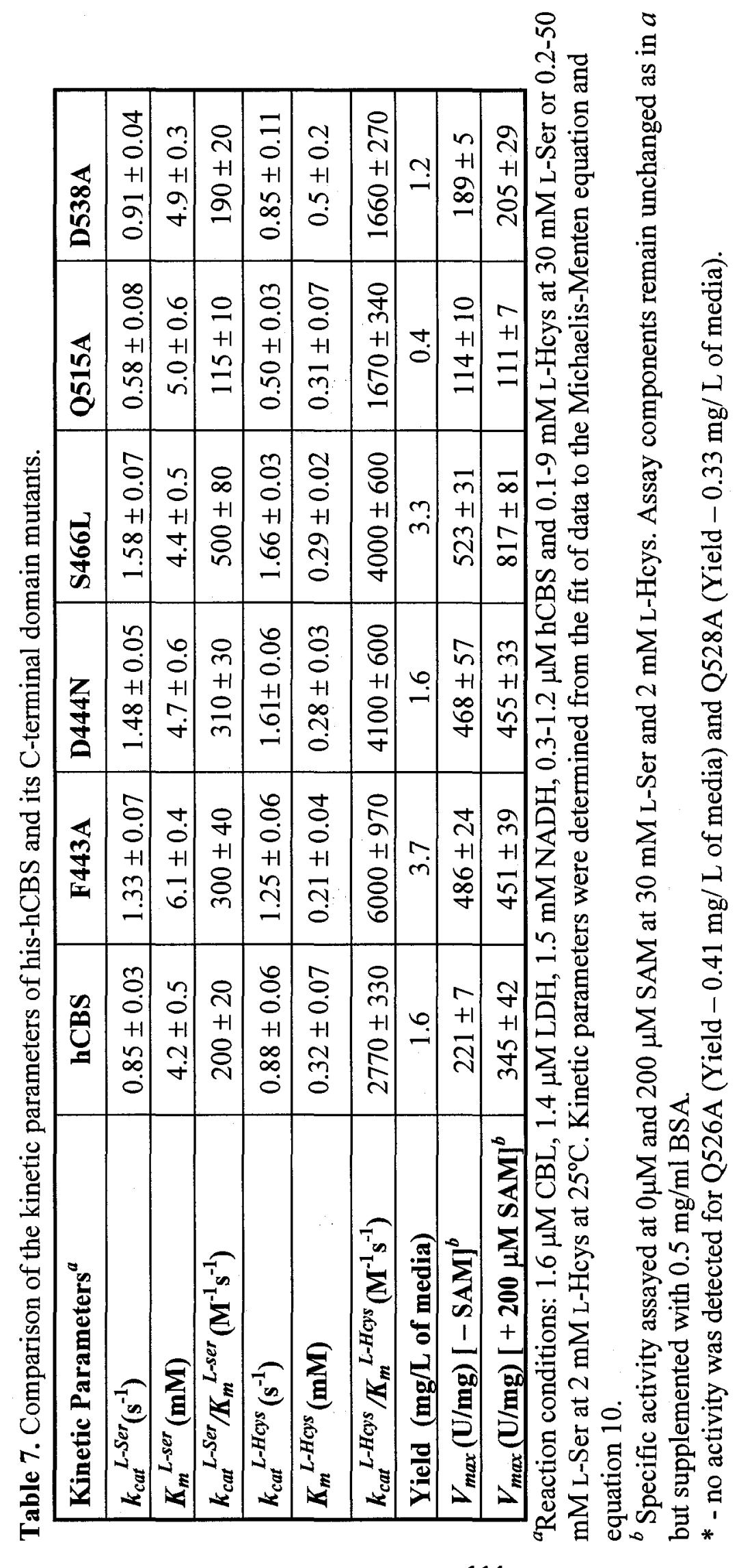




\subsection{Discussion.}

The CBS-domain pair from cystathionine $\beta$-synthase has potential interacting residues for SAM situated in the cleft between the two domains. Deletion of the CBS domains in the yeast and human enzymes leads to formation of highly active dimers, which are unresponsive to SAM, and highlights the autoinhibitory nature of CBS domains [Shan and Kruger, 1994; Kery et al., 1998; Evande et al., 2002]. In the native human enzyme, SAM binding is accompanied by a conformational change that increases the activity of CBS by 2-to 3-fold [Kery et al., 1998; Taoka et al., 1998; Frank et al., 2006; Frank et al., 2008]. Since, the full-length crystal structure of CBS is unavailable, a series of site-directed mutants of residues in the regulatory domain of CBS were constructed to probe their roles in SAM binding. These residues were targeted based on sequence alignments and the structures of CBS domains from other proteins, including AMPK and ClC-5.

\subsubsection{Characterization of F443A and D538A (hCBS).}

Hydrophobic and hydrogen-bonding interactions with the adenine and ribose rings of adenine ligands are observed for $\mathrm{ClC}-5$ and AMPK. The Y617A mutant of ClC-5 abolishes ATP binding and changes the transport of $\mathrm{Cl}^{-}$[Meyer et al., 2007]. Residue F443 (hCBS), in the CBS1 domain, corresponds to Y617 of ClC-5, which interact via a $\pi$-stacking interaction with the adenine group of ATP. The hCBS-F443A mutant has increased activity, as evidenced by its 1.6 -fold and 1.5-fold increases in $k_{\text {cat }}^{\mathrm{L}-\mathrm{Ser}}$ and $k_{\mathrm{cat}}^{\mathrm{L}-}$ Hcys, respectively (table 7). A 2.2- fold increase in specific activity, compared to the wild type, is observed for the SAM-insensitive F443A mutant. A previous study demonstrated 
that the homocystinuria-associated D444N mutant of the adjacent residue is locked in an activated conformation [Sen et al., 2005]. These results suggest that residues F443 and D444 are involved in both SAM binding and the intersubunit communication required for allosteric activation.

First characterized by Day et al., [2007] in AMPK, the putative ribose binding domain is observed in both CBS domains of hCBS and yCBS. The conserved aspartate residue at the C-terminus of this motif corresponds to D727, D317 and D538 of ClC-5, AMPK and hCBS (CBS2 domain), respectively. In ClC-5, the D727A mutation eliminates H-bonds with the hydroxyl moieties of the ATP ribose sugar and in the structure of the AMPK-AMP complex D317 is observed to hydrogen bonds with the ribose hydroxyl groups [Meyer et al., 2007]. The hCBS D538A mutant has a similar specific activity (189 $\pm 5 \mathrm{U} / \mathrm{mg}$ ) as the wild type enzyme, but is not responsive to SAM (table 7), suggesting that residue D538 of hCBS is involved in SAM binding but not in intersubunit communication.

\subsubsection{Mutation of conserved glutamine residues impairs hCBS folding/activity.}

The four targeted glutamine were chosen because of their conservation among eukaryotic CBS and their situation within the SAM-protected peptide (VVHEQIQYHSTGKSSQRQMVF) identified from hydrogen exchange and peptide mass mapping [Sen et al., 2005]. The mild reduction in both ${k_{\text {cat }}}^{\text {L-Ser }}(1.5-$ fold $)$ and $k_{\text {cat }}^{\text {L-Hcys }}(1.7-$ fold $)$ values, combined with the lack of activation by SAM of the hCBS-Q515A mutant suggests that Q515 is involved in intersubunit communication, but not in SAM binding. The yield of Q515A was similar to the Q526A (0.41 mg/ L of media) and Q528A (0.33 
$\mathrm{mg} / \mathrm{L}$ of media) mutants, but the latter two enzymes do not display measurable levels of activity. The Q517A mutant was insoluble. The inactive mutants, Q526A and Q528A, and the insoluble Q517A mutant suggest the critical role of these residues in maintaining the structural integrity of the enzyme.

\subsubsection{Characterization of D444N and S466L (hCBS).}

Residue D444 of the CBS1 domain of hCBS aligns with S226 of AMPK and S618 of ClC-5, residues that hydrogen bind to the $\alpha$-, and $\gamma$ - phosphate, respectively, of the adenine nucleotides [Figure 6; Meyer et al., 2007; Day et al., 2007]. In CLC-5, the point mutation, S618A, has no effect on ATP binding [Meyer et al., 2007]. In contrast, the SAM affinity of the D444N mutant of hCBS is reduced by $\sim 15$-fold, compared to the wild-type enzyme [Kluijtmans et al., 1996; Scott et al., 2004].

The homocystinuria-associated D444N and S466L mutants of hCBS have been previously investigated and were included to serve as experimental controls in the expression and analysis of site-directed mutants of hCBS. The $k_{\mathrm{cat}}{ }^{\mathrm{L}-\mathrm{Ser}}$ values of the D444N and S466L homocystinuria-associated mutations are increased by 1.7 and 1.9fold, respectively, while the $K_{\mathrm{m}}$ values for both substrates are unchanged (table 7). The specific activity of the D444N mutant is unaffected by $200 \mu \mathrm{M}$ SAM, consistent with the report by Evande et al., [2002] that the $K_{\text {act }}^{\text {SAM }}$ value for the mutant is $460 \pm 130 \mu \mathrm{M}$. Therefore, the lower SAM concentration used in the assay was insufficient to observe any change in activity. The S466 $\mathrm{L}$ mutant is also constitutively activated, but was activated a further 1.6-fold by $200 \mu \mathrm{M}$ SAM (table 7). This contrasts the report by Janosik et al. [2001] that although the SAM-binding of the S466L mutant is unresponsive to SAM. The 
difference between the current results and those reported by Janosik et al., [2001] may be due to the N-terminal 23-residue linker, retained after proteolytic cleavage of its GST fusion partner, that is not present in the 6-His/hCBS enzyme.

\subsubsection{Yeast CBS is SAM insensitive.}

The observation that yCBS is not activated by SAM contradicts Ono et al. [1994], but is in agreement with more recent studies [Jhee et al., 2000b; Maclean et al., 2000]. The yCBS mutants were constructed to assess the inter-domain communication between the CBS domains and the catalytic core of the enzyme. The $k_{\text {cat }}{ }^{\mathrm{L}-S e r}\left(2.9 \pm 0.2 \mathrm{~s}^{-1}\right)$ of yCBS-D399A, which corresponds to the D444N mutant of hCBS, is comparable to the wild type enzyme. This contrasts with the 2.2 -fold increase in specific activity of the hCBS-D444N mutant and suggests how the yeast mutation does not induce structural changes that result in enzyme activation. The other yCBS residues targeted correspond to positions in the putative ribose-binding motif of the first and second CBS domains of yCBS (CBS1 - T414A, S416A, and E417A and CBS2 - T498A and D501A). With the exception of D501A, the $k_{\text {cat }}^{\text {L-Ser }}$ and $K_{\mathrm{m}}^{\mathrm{L}-\mathrm{Ser}}$ values are unaffected (Table 6). Urea denaturation qualitatively shows the stability of the yeast mutants as similar, although E417A has a slightly higher stability than the wild type yCBS enzyme.

\subsubsection{Future work.}

Although the specific activity of D444N is twice as high as the wild type, its relation to homocystinuria is not based on cellular activity. Rather, fibroblast cell lines show a 4-fold decrease in the steady-state levels of D444N CBS and unaltered mRNA 
levels [Evade et al., 2002]. Similarly, transgenic mice expressing hCBS-S466L elevated mRNA levels but suffer from variably reduced steady-state levels [Gupta et al., 2008]. Interestingly, an unphysiological concentration of ATP (5 mM) was shown to increase the specific activity of hCBS, but not yCBS, by $\sim 2$ - fold. This suggests that saturating concentrations of adenine and ribose sugar moieties of ATP are bound by the CBS regulatory domain and induce conformational changes associated with enzyme activation. Future work will determine the $K_{\text {act }}$ and $K_{\mathrm{d}}$ for this nucleotide, enabling comparison with the corresponding values for SAM. The ability of the hCBS site-directed mutants to bind SAM and the role of specific interactions will be investigated via isothermal titration calorimetry. 


\section{CONCLUSIONS.}

The reverse transsulfuration pathway prevents the buildup of the toxic metabolite L-Hcys in the cellular environment. Cystathionine $\beta$-synthase condenses L-Hcys and LSer in the first step of this pathway, which ultimately produces L-Cys, the precursor of glutathione biosynthesis. The methionine pool is also maintained by L-Hcys remethylation and the flux between the transsulfuration and remethylation pathways is regulated by the ubiquitous cellular methyl donor, SAM.

The susceptibility of human CBS to proteolysis and aggregation hinders the recombinant expression of this enzyme. Current methods employ multiple chromatographic steps and a protease-cleavable fusion partner. The aim of Section 9 was to reduce the cost and increase the efficiency of hCBS purification by the use of a single affinity (Ni-NTA) chromatography step. Five expression constructs were made (6-HisGST/hCBS, 6-His-GFP/hCBS, 6-His-linker/hCBS, 6-His/hCBS and hCBS/6-His) and it was determined that an affinity tag is sufficient for the purification of soluble hCBS, thereby eliminating the need for a fusion partner. Furthermore, as the $k_{\mathrm{cat}} / K_{\mathrm{m}}{ }^{\mathrm{L}-S e r}$ and $k_{\text {cat }} / K_{\mathrm{m}}^{\text {L-Hcys }}$ values are not altered by the presence of an N-terminal, 6-His affinity tag, showing that its removal is not required. The steady-state kinetic parameters of the $\beta$ replacement activity of 6-His/hCBS and 6-His-linker/hCBS were determined using a continuous assay that enabled global analysis and the detection of previously-unreported substrate inhibition by L-Hcys. Future work will focus on further reducing the cost of hCBS expression by eliminating the need supplementation of growth media with the heme precursor, $\delta$-aminolevulinic acid, by making an expression construct carrying genes for both aminolevulinate synthase and 6-His/hCBS. 
The catalytic versatility of the PLP cofactor is regulated by the enzyme to which it is bound and the properties of the cofactor are governed by interactions with active site residues. Residue S289 of yCBS forms a hydrogen bond with N-1 of PLP and the importance of this interaction was determined by investigation of the S289A and S289D site-directed mutants. The lack of activity of the S289D mutant and the $\sim 800$-fold reduction in the $k_{\mathrm{cat}} / K_{\mathrm{m}}{ }^{\mathrm{L}-\mathrm{Ser}}$ of S289A suggest that the yCBS-catalyzed $\alpha, \beta$-elimination of L-Ser occurs via an $\mathrm{E}_{1}$ mechanism. Fluorescence energy transfer from tryptophan residues of the enzyme to the PLP cofactor is reduced in S289A and absent in S289D. These observations demonstrate that S289 plays a critical role in maintaining the properties and the orientation of the cofactor. Future studies will investigate the role of other active-site residues in regulating cofactor chemistry in yCBS. For example, the ring hydroxyl group of the PLP cofactor interacts, via a hydrogen bond, with the side chain of N84, which likely also interacts with the $\alpha$-carboxylate of the L-Ser substrate. Investigation of site-directed mutants of N84 will enable the role of this residue in the yCBS active site to be defined.

The CBS domains that are situated in the regulatory domain of CBS have also been observed in other proteins with diverse functions. CBS domains, such as those of IMPDH, AMPK and ClC-5, show a similar fold and capacity to bind adenine ligands, despite low sequence conservation. Characteristic features of these CBS domains include hydrophobic interactions with the adenine ring and a ribose-binding motif. A series of site-directed mutants were constructed to identify residues involved in SAM binding and inter-domain communication in the SAM-insensitive yCBS and the SAM-responsive hCBS. The loss of responsiveness to SAM of the F443A, D538A and the 
homocystinuria-associated D444N mutants suggests diminished ligand binding. The specific activity of the F443A and D444N mutants is similar to that of the SAM-activated wild-type enzyme, suggesting that these residues also play a role in the intersubunit communication involved in allosteric activation. In contrast, the D339A mutant of yCBS, which corresponds to residue D444 of hCBS, has similar $k_{\text {cat }}{ }^{\mathrm{L}-\mathrm{Ser}}$ and $k_{\text {cat }}^{\mathrm{L}-\mathrm{Hcys}}$ values as the wild type yeast enzyme. This demonstrates a difference in the intersubunit communication between the yeast and human enzymes. Future work will investigate the effect of these mutants on SAM binding via isothermal titration calorimetry. 


\section{REFERENCES.}

Adams, E. (1969). Fluorimetric determination of pyridoxal phosphate in enzymes. Anal Biochem. 31: 118-122.

Aitken, S.M., and Kirsch, J. F. (2003). Kinetics of the Yeast Cystathionine $\beta$-Synthase Forward and Reverse Reactions: Continuous Assays and the Equilibrium Constant for the Reaction. Biochemistry. 42:571- 578.

Aitken, S.M., and Kirsch, J. F. (2004). Role of Active-Site Residues Thr81, Ser82, Thr85, Gln157, and Tyr158 in Yeast Cystathionine $\beta$-Synthase Catalysis and Reaction Specificity. Biochemistry 43: 1963 - 1971.

Aitken S.M. and Kirsch, J. F. (2005). The enzymology of cystathionine biosynthesis: strategies for the control of substrate and reaction specificity. Arch Biochem Biophys. 433:166-175

Al-Essa M, Rashed M, Ozand PT. (1998). Saudi experience with classic homocystinuria. Ann Saudi Med. 18: 230-233.

Banerjee R, Zou CG. (2005). Redox regulation and reaction mechanism of human cystathionine-beta-synthase: a PLP-dependent hemesensor protein. Arch Biochem Biophys. 433:144-56

Bateman, A. (1997). The structure of a domain common to archaebacteria and the homocystinuria disease protein. Trends Biochem. Sci. 22:12-13.

Bennetts, B., Rychkov, G.Y.,Ng , H.-L.,Morton, C.J.,Stapleton, D.,Parker, M.W., Cromer, B.A. (2005). Cytoplasmic ATP-sensing domains regulate gating of skeletal muscle ClC-1 chloride channels. J. Biol. Chem. 280: $32452-32458$.

Bennetts, B., Parker, M.W., Cromer, B.A. (2007). Inhibition of skeletal muscle CLC-1 chloride channels by low intracellular pH and ATP. J. Biol. Chem. 282:3278032791 .

Berg, J.M., Tymoczko, J.L., and Stryer, L. (2002). Biochemistry. New York.

Blair, E, Redwood, C, Ashrafian, H, Oliveira, M, Broxholme, J, Kerr, B, Salmon, A, Ostman-Smith, I, and Watkins, H. (2001). Mutations in the $\gamma 2$ subunit of AMPactivated protein kinase cause familial hypertrophic cardiomyopathy: evidence for the central role of energy compromise in disease pathogenesis. Hum Mol Genet 10: $1215-1220$

Borcsok, E. and Abeles, R. H. (1982). Mechanism of action of cystathionine synthase, Arch Biochem Biophys. 213: 695-707. 
Bowne SJ, Sullivan LS, Blanton SH, Cepko CL, Blackshaw S, Birch DG, HughbanksWheaton D, Heckenlively JR, and Daiger SP. (2002). Mutations in the inosine monophosphate dehydrogenase 1 gene (IMPDH1) cause the RP10 form of autosomal dominant retinitis pigmentosa. Hum Mol Genet. 11: 559-568.

Bradford, M.M. (1976). A rapid and sensitive method for the quantitation of microgram quantities of protein utilizing the principle of protein-dye binding. Anal. Biochem. 72: 248-254.

Braunstein, A. E., and Goryachenkova, E. V. (1984). Adv. Enzymol. 56: 1-89

Bukovska, G., Kery, V., and Kraus, J.P. (1994). Expression of human cystathionine betasynthase in Escherichia coli: purification and characterization. Protein Expression and Purification. 5, 442-448.

Burkhard, P., Rao, G. S., Hohenester, E., Schnackerz, K. D., Cook, P. F., Jansonius, J. N. (1998). Three-dimensional structure of O-acetylserine sulfhydrylase from Salmonella typhimurium. J Mol Biol. 283: 121-133.

Burstyn, J.N., Kraus, J.P., Rodgers, K.R., Clark, R.W., Park, R.B., Rees, K.A., Oliveriusovai, J., Lukat-Rodegers, Gudrun, S. and Pazieni, S. (2004). The redox behavior of the heme in cystathionine $\beta$-synthase is sensitive to $\mathrm{pH}$. Biochemistry. 43:14684-14695

Cherney, M.M., Pazicni, S., Frank, N., Marvin, K.A., Kraus, J.P., and Burstyn, J.N. (2007). Ferrous human cystathionine beta-synthase looses activity during enzyme assay due to a ligand switch process. Biochemistry. 46, 13199-13210.

Christen, P. \& Mehta, P.K. ( 2001) From cofactor to enzymes. The molecular evolution of pyridoxal-5'-phosphate-dependent enzymes. Chem. Rec. 1, 436-447

Clarke, R., Smith, A.D., Jobst, K.A., Retsum, H., Sutton, L. and Ueland, P.M. (1998). Folate, vitamin $\mathrm{B}_{12}$, and serum total homocysteine levels in confirmed Alzheimer disease. Arch. Neurol. 55:1449-1455.

Cook, P. F. 2003. $\alpha, \beta$-elimination reaction of $O$-acetylserine sulfhydrylase: is the pyridine ring required? Biochim. Biophys. Acta. 1647: 66-69.

Cook, P. F. and Wedding, R. T. A reaction mechanism from steady-state kinetic studies for O-acetylserine sulfhydrylase from Salmonella typhimurium LT-2. (1976). $J$ Biol Chem. 251: 2023-2029.

Daum, S., Tai, C.H., and Cook, P.F. 2003. Characterization of the S272A,D site-directed mutations of $O$-acetylserine sulfhydrylase: involvement of the pyridine ring in the $\alpha, \beta$-elimination reaction. Biochem. 42:106-113. 
Day P, Sharff A, Parra L, Cleasby A, Williams M, Horer S, Nar H, Redemann N, Tickle I, Yon J. (2007). Structure of a CBS-domain pair from the regulatory gammal subunit of human AMPK in complex with AMP and ZMP. Acta Crystallogr D Biol Crystallogr. 63:587-96.

De Franchis R, Kozich V, McInnes RR, Kraus JP (1994) Identical genotypes in siblings with different homocystinuric phenotypes: identification of three mutations in cystathionine $\beta$ - synthase using an improved bacterial expression system. Hum Mol Genet. 3: 1103-1108

DeLano, W.L. (2002). The PyMOL Molecular Graphics System. DeLano Scientific, San Carlos, CA, USA.

Drewe, W. F., Jr., and Dunn, M. F. (1985). Detection and identification of intermediates in the reaction of L-serine with Escherichia coli tryptophan synthase via rapidscanning ultraviolet-visible spectroscopy. Biochemistry. 24: 3977-3987.

Dunathan, H.C. (1966). Conformation and reaction specificity in pyridoxal phosphate enzymes. Proc. Natl. Acad. Sci. U.S.A. 55: 712-716.

Dunathan, H. C. (1971). Stereochemical aspects of pyridoxal phosphate catalysis. $A d v$ Enzymol Relat Areas Mol Biol. 35: 79-134.

Dutzler R, Campbell EB, Cadene M, Chait BT, and MacKinnon R. (2002). X-ray structure of a $\mathrm{ClC}$ chloride channel at 3.0A reveals the molecular basis of anion selectivity. Nature. 415: 287-294.

El-Said, M.F., Badii, R., Bessisso, M.S., Shahbek, N., El-Ali, M.G., El-Marikhie, M., ElZyoid, M., Salem, M.S., Bener, A., Hoffmann, G.F., Zschocke, J. (2006). A common mutation in the CBS gene explains a high incidence of homocystinuria in the Qatari population. Hum Mutat. 27: 719-725.

Eliot, A.C. and Kirsch, J.F. (2004). Pyridoxal phosphate enzymes: mechanistic, structural and evolutionary considerations. Annu. Rev. Biochem. 73: 383-415.

Ellman G.L. (1959). Tissue sulfhydryl groups. Arch. Biochem. Biophys. 82: 70-77.

Evande R, Blom H, Boers GH, Banerjee R.. (2002). Alleviation of intrasteric inhibition by the pathogenic activation domain mutation, $\mathrm{D} 444 \mathrm{~N}$, in human cystathionine beta-synthase. Biochemistry. 41:11832-7.

Finkelstein, JD, Kyle, KE, Martin, JJ, and Pick, AM. (1975). Activation of cystathionine $\beta$-synthase by adenosylmethionine and adenosylethionine, Biochem. Biophys. Res. Commun. 66: 81-87. 
Finkelstein, J.D. and Martin, J.J. (1984). Methionine metabolism in mammals. Distribution of homocysteine between competing pathways. J. Biol. Chem. 15: $9508-9513$

Finkelstein, J.D. (1990). Methionine metabolism in mammals. J. Nutr. Biochem. 1: 228237

Fisher, C.L. and Pei, G.K. Modification of a PCR-based site-directed mutagenesis method. (1997). Biotechniques.23: 570-1, 574.

Frank, N., Kery, V. Maclean, K.N. and Kraus, JP. (2006). Solvent-Accessible Cysteines in Human Cystathionine $\beta$-Synthase: Crucial Role of Cysteine 431 in SAdenosyl-L-methionine Binding. Biochemistry. 45: 11021-11029.

Frank, N., Kent, J.O., Meier, M. and Kraus J.P. (2008). Purification and characterization of the wild type and truncated human cystathionine $\beta$-synthase enzymes expressed in E. coli. Arch. Biochem. Biophys. 470, 64-72.

Gallagher, P.M, Ward P, Tan S, Naughten E, Kraus JP, Sellar GC, McConnell DJ, Graham I, Whitehead AS. (1995). High frequency (71\%) of cystathionine betasynthase mutation G307S in Irish homocystinuria patients. Human Mutat. 6:17780 .

Goldberg, J. M., Swanson, R. V., Goodman, H. S., and Kirsch, J.F. (1991). The Tyrosine225 to Phenylalanine Mutation of Escherichia coli Aspartate Aminotransferase Results in an Alkaline Transition in the Spectrophotometric and Kinetic pKa Values and Reduced Values of both $\mathrm{k}_{\text {cat }}$ and $\mathrm{K}_{\mathrm{m}}$. Biochemistry 30, 305-312.

Hajjar, A.K. (2001). Homocysteine: a sulph'rous fire. The Journal of Clinical Investigation. Volume 107: 6:663-4

Hardie, D. G., Carling, D. \& Carlson, M. (1998). Annu. Rev. Biochem. 67:821-855.

Hardie, D. G., Scott, J. W., Pan, D. A. \& Hudson, E. R. (2003). FEBS Lett. 546, 113-120.

Higuchi, R. 1990. In PCR protocols: a guide to methods and applications, in Innis, M. A., Gelfand, D. H., Sninsky, J. J., and White, T. J., (Eds.), Academic Press, San Diego, 1990, pp. 177-183.

Hyde, C. C., Ahmed, S. A., Padlan, E. A., Miles, E. W., Davies, D. R. (1988). Threedimensional structure of the tryptophan synthase alpha 2 beta 2 multienzyme complex from Salmonella typhimurium. J Biol Chem. 263: 17857-17871.

Janosik, M., Meier, M., Kery, V., Oliveriusova, J., Burkhard, P., and Kraus, J.P. (2001a). Crystallization and preliminary X-ray diffraction analysis of the active core of 
human recombinant cystathionine beta-synthase: an enzyme involved in vascular disease. Acta Crys. D. 57, 289-291.

Janosik, M., Kery, V., Gaustadnes, M., Maclean, K.N., and Kraus, J.P. (2001b).

Regulation of human cystathionine $\beta$-synthase by $S$-adenosyl-L-methionine: evidence for two catalytically active conformations involving an autoinhibitory domain in the C-terminal region. Biochemistry. 40: 10625-10633.

Jentsch, T.J., Steinmeyer, K., Schwarz, G. (1990). Primary structure of Torpedo marmorata chloride channel isolated by expression cloning in Xenopus oocytes. Nature. 348: 510-514.

Jentsch TJ. (1996). Chloride channels: a molecular perspective. Curr Opin Neurobiol. 6: $303-310$.

Jentsch, T.J., Friedrich, T., Schriever, A., Yamada, H. (1999). The CLC chloride channel family. Pflugers Arch. 437: 783-795.

Jentsch, T.J., Stein, V., Weinreich, F., and Zdebik, A.A. (2002). Molecular structure and physiological function of chloride channels. Physiol Rev. 82: 503-568.

Jhee, K. H., Yang, L. H., Ahmed, S. A., McPhie, P., Rowlett, R., Miles, E. W. (1997) Protein Sci. 6: 67.

Jhee, K.H., Yang, L.H., Ahmed, S.A., McPhie, P., Rowlett, R., and Miles, E.W. (1998a). Mutation of an active site residue of tryptophan synthase (beta-serine 377 ) alters cofactor chemistry. J. Biol. Chem. 273: 11417-11422.

Jhee, K. H. , McPhie, P., Ro, H. S., Miles, E. W. (1998b). Tryptophan synthase mutations that alter cofactor chemistry lead to mechanism-based inactivation. Biochemistry. $37: 14591-14604$.

Jhee, K.H., McPhie, P., and Miles, E.W. (2000a). Yeast cystathionine $\beta$-synthase is a pyridoxal phosphate enzyme but, unlike the human enzyme, is not a heme protein. J. Biol. Chem. 275: 11541-11544.

Jhee, K.H., McPhie, P., and Miles, E.W. (2000b). Domain architecture of the hemeindependent yeast cystathionine $\beta$-synthase provides insights into mechanisms of catalysis and regulation. Biochemistry. 39: 10548-10556.

Jhee, K.H., Niks, D., McPhie, P., Dunn, M.F., and Miles, E.W. (2001). The reaction of yeast cystathionine $\beta$-synthase is rate-limited by the conversion of aminoacrylate to cystathionine. Biochem. 40: 10873-10880. 
Kabil, O. and Banerjee, R. (1999). Deletion of the regulatory domain in the pyridoxal phosphate-dependent heme protein cystathionine $\beta$-synthase alleviates the defect observed in a catalytic site mutant. J. Biol. Chem. 274:31256-31260

Kabil, O., Taoka, S., LoBrutto, R., Shoemaker, R., and Banerjee, R. (2001). Pyridoxal phosphate binding sites are similar in human heme-dependent and yeast hemeindependent cystathionine beta-synthases. Evidence from 31P NMR and pulsed EPR spectroscopy that heme and PLP cofactors are not proximal in the human enzyme. J. Biol. Chem. 276, 19350-19355.

Kahn, B. B., Alquier, T., Carling, D. \& Hardie, D. G. (2005). Cell Metab. 1: 15-25.

Kamoun, P. (2001). Mental retardation in Down syndrome: a hydrogen sulfide hypothesis. Med. Hypotheses. 57: 389-92.

Kashiwamata, S. and Greenberg, D.M. (1970). Studies on cystathionine synthase of rat liver. Properties of the highly purified enzyme. Biochim Biophys Acta. 488-500.

Kaur, M., Kabra, M., Das, G.P., Suri, M, Verma, I.C. (1995). Clinical and biochemical studies in homocystinuria. Indian Pediatr. 10: 1067-75.

Kemp, B. E., Stapleton, D., Campbell, D. J., Chen, Z. P., Murthy, S., Walter, M., Gupta, A., Adams, J. J., Katsis, F., van Denderen, B., Jennings, I. G., Iseli, T., Michell, B. J. \& Witters, L. A. (2003). Biochem. Soc Trans. 31: 162-168.

Kemp, B.E. (2004). Bateman domains and adenosine derivatives form a binding contract. J. Clin. Invest. 113: 182-184.

Kennan, A., Aherne, A., Palfi, A., Humphries, M., McKee, A., Stitt, A., Simpson, D.A., Demtroder, K., Orntoft, T., Ayuso, C., Kenna, P.F., Farrar, G.J., and Humphries, P. (2002). Identification of an IMPDH1 mutation in autosomal dominant retinitis pigmentosa (RP10) revealed following comparative microarray analysis of transcripts derived from retinas of wild-type and Rho mice. Hum Mol Genet 11: $547-557$.

Kery, V. Bukovska, G., Kraus, J.P. (1994). Transsulfuration depends on heme in addition to pyridoxal 5'-phosphate. J. Biol. Chem. 269:25283-25288.

Kery, V., Elleder, D., and Kraus, J.P. (1995). Delta-aminolevulinate increases heme saturation and yield of human cystathionine beta-synthase expressed in Escherichia coli. Arch. Biochem. Biophys. 316, 24-29.

Kery, V., Poneleit, L, Kraus, JP. (1998). Trypsin cleavage of human cystathionine $\beta$ synthase into an evolutionarily conserved active core: structural and functional consequences. Arch Biochem Biophys. 355: 222-232. 
Kluijtmans, L.A., Boers, G.H., Stevens, E.M. ,Renier, W.O., Kraus, J.P., Trijbels F.J., Heuvel, L.P., and Blom, H.J. (1996). Defective cystathionine $\beta$-synthase regulation by $S$-adenosylmethionine in a partially pyridoxine responsive homocystinuria patient. $J$ Clin Invest . 98: 285-289.

Kozich, V., Janosik, M., Sokolova, J., Oliveriusova, J., Orendac, M., Kraus, J. P. and Elleder, D. (1997). Analysis of CBS alleles in Czech and Slovak patients with homocystinuria: Report on three novel mutations E176K, W409X and $1223+37$ de199. J Inher Metab Dis. 20: 363-366.

Kraus, J., Packman, P.S., Fowler, B., and Rosenberg, L.E. (1978). Purification and properties of cystathionine $\beta$-synthase from human liver. Evidence for identical subunits. J. Biol. Chem. 253: 6523-6528.

Kraus, J.P, and Rosenberg, L.E. (1983). Cystathionine $\beta$-synthase from human liver: Improved purification scheme and additional characterization of the enzyme in crude and pure form. Arch. Biochem. Biophys. 222: 44-52.

Kraus, J.P. (1994). Molecular basis of phenotype expression in homocystiniuria. J. Inher. Metab. Dis. 17: 383-390.

Kraus, J.P., Janosik, M., Kozich, V., Mandell, R., Shih, V., Sperandeo, M.P., Sebastio, G., de Franchis, R., Andria, G., Kluijtmans, L.A.J., Blom, H., Boers, G.H.J., Gordon, R. B., Kamoun, P., Tsai, M.Y., Kruger, W.D., Koch, H.G., Ohura, T., and Gaustadnes, M. (1999). Cystathionine $\beta$-synthase mutations in homocystinuria. Human Mutations. 13: 362-375.

Kruger, W.D., Liqun, W., Kwang-Hwan, J., Xiang, H., Patricia, M.D. and Donald, W.J. (2004). Modulation of cystathionine $\beta$-synthase level regulates total homocysteine in mice. American Heart Association. 94: 1318-1324

Ling, Q., and Hajjar, K.A. (2000). Inhibition of endothelial cell thromboresistance by homocysteine. J. Nutr. 130: 373-376.

Maclean, K.N., Janosik, M., Oliveriusova, J., Kery, V., Kraus J.P. (2000).

Transsulfuration in Saccharomyces cerevisiae is not dependent on heme: purification and characterization of recombinant yeast cystathionine $\beta$-synthase. Journal of Inorganic Biochemistry. 3: 161-171.

Maclean KN, Gaustadnes M, Oliveriusova J, Janosik M, Kraus E, Kozich V, Kery V, Skovby F, Rudiger N, Ingerslev J, Stabler SP, Allen RH, Kraus JP. (2002). High homocysteine and thrombosis without connective tissue disorders are $\square$ associated with a novel class of cystathionine beta-synthase (CBS) mutations. Hum Mutat. 19(6): 641-655 
Maduke, M., Pheasant, D.J., and Miller, C. (1999). High-level expression, functional reconstitution, and quaternary structure of a prokaryotic ClC-type chloride channel. J Gen Physiol. 114: 713-722.

Maduke, M., Miller, C., Mindell, J.A. A decade of CLC chloride channels: structure, mechanism, and many unsettled questions. Annu Rev Biophys Biomol Struct. (2000). 29:411-438.

McCully, S.K. (2001). The biomedical significance of homocysteine. Journal of scientific exploration. 15: 5-20.

McClure, G. D. Jr., and Cook, P. F. (1994). Product binding to the alpha-carboxyl subsite results in a conformational change at the active site of $\mathrm{O}$-acetylserine sulfhydrylase-A: evidence from fluorescence spectroscopy. Biochemistry. 33: 1674-1683.

Mehta, P. K. and Christen, P. (2000). The molecular evolution of pyridoxal-5'-phosphatedependent enzymes. Adv Enzymol Relat Areas Mol Biol.74: 129-184.

Meier, M., Kery, V., Janosik, M., Kraus, J.P. Burkhard, P. (2001). Structure of human cystathionine $\beta$-synthase: a unique pyridoxal 5'-phosphate-dependent heme protein. $E M B O$ J. 20: 3910-3916.

Meier, M., Oliveriusora J., Kraus J.P. Burkhard, P. (2003). Structural insights into mutations of cystathionine $\beta$-Synthase. Biochem. Biophys. Acta. 1647: 206-213.

Meyer, S., Savaresi, S., Forster, I.C., Dutzler, R. (2007). Nucleotide recognition by the cytoplasmic domain of the human chloride transporter ClC-5. Nat Struct Mol Biol. 1: 60-67.

Miles, E.W. (1986). Pyridoxal Phosphate: Chemical, Biochemical and Medical Aspects. Dolphin, D., Poulson, D., and Avramovic, O., eds. John Wiley and Sons, New York. 1: 253-310.

Miles, E. W. (2001). Tryptophan synthase: a multienzyme complex with an intramolecular tunnel. Chem Rec. 1: 140-151.

MOE. (2007). Molecular Operating Environment, Chemical Computing Group, Montreal.

Morrison, M., and Horic. S. 1965. Anal. Biochem. 12, 77-82.

Mosharov, E., Cranford, M.R., Banerjee, R. (2000). The quantitatively important relationship between homocysteine metabolism and glutathione synthesis by the transsulfuration pathway and its regulation by redox changes. Biochemistry. 39: 13005- 13011. 
Mozzarelli, A, Bettati, S. (2006). Exploring the pyridoxal 5'-phosphate-dependent enzymes. Chem Rec. 2006;6(5):275-87.

Mudd, S.H., Dkovby, F., Levy, H.L., Pettigrew, K.D., Wilcken, B. and Pyeritz, R.E. (1985). The natural history of homocystinuria due to cystathionine $\beta$-synthase. Am. J. Hum. Genet. 37: 1-31

Mudd, S.H., Levy, H. L., and Skovby, F. (1989) in The Metabolic Basis of Inherited Disease (Scriver, C. R., Beaudet, A. L., Sly, W. S., and Valle, D., eds) pp. 693734, McGraw-Hill, New York

Mudd, H., Levy, H.L., and Skovby, F. (2001). Disorders of transsulfuration. In The metabolic and molecular basis of inherited disease. C.R. Scriver, A.L. Beaudet, W.S. Sly, and D. Valle, editors. McGraw-Hill Companies, New York p. 2007.

Munke, M., Kraus, J.P., Ohura, T., and Francke, U. (1988). The gene for cystathionine beta-synthase (CBS) maps to the subtelomeric region on human chromosome21Q and to proximal mouse chromosome-17. Am. J. Hum. Gen. 42, 550-559.

Nozaki, T., Shigeta, Y., Saito-Nakano, Y., Imada, M., and Kruger, W. D. (2001). J. Biol. Chem. 276: 6516-6523.

Oliveriusova, J., Kery, V., Maclean, K.N., and Kraus, J.P. (2002). Deletion mutagenesis of human cystathionine beta-synthase. Impact on activity, oligomeric status, and S-adenosylmethionine regulation. J. Biol. Chem, 10:1074

Pazicni, G. S., Lukat-Rodgers, J.Oliveriusova, K.A.Rees, R. B. Parks, R.W. Clark, K. R.Rodgers, J. P. Kraus and J.N. Burstyn. (2004). Biochemistry. 43: 14684-14690.

Pazicni, S., Cherney, M.M., Lukat-Rodgers, G.S., Oliveriusova, J., Rodgers, K.R., Kraus, J.P., and Burstyn, J.P. (2005). The heme of cystathionine beta-synthase likely undergoes a thermally induced redox-mediated ligand switch. Biochemistry. 44: 16785-16795.

Porto, M.P., Galdieri, L.C., Pereira, V.G., Vergani, N., da Rocha, J.C., Micheletti, C., Martins, A.M., Perez, A.B., Almeida, V.D. (2005). Molecular analysis of homocystinuria in Brazilian patients. Clin Chim Acta. 362:71-78.

Ponting, C.P. (1997). CBS domains in CIC chloride channels implicated in myotonia and nephrolithiasis (kidney stones). J. Mol. Med. $75: 160-163$.

Prudova A, Bauman Z, Braun A, Vitvitsky V, Lu SC, Banerjee R. (2005). Sadenosylmethionine stabilizes cystathionine $\beta$-synthase and modulates redox capacity. Proc Natl Acad Sci; 103: 6489-6494 
Refsum H, Fredriksen A, Meyer K, Ueland PM, Kase BF. (2004). Birth prevalence of homocystinuria. $J$ Pediatr. 144: 830-2.

Rufer, A.C., Thiebach, L.,Baer, K., Klein, H.W., Hennig, M. X-ray structure of glutathione S-transferase from Schistosoma japonicum in a new crystal form reveals flexibility of the substrate-binding site. (2005). Acta Crystallogr Sect F Struct Biol Cryst Commun.61: 263-265.

Saunders, W. (1976). Distinguishing between concerted and nonconcerted eliminations. Acc Chem Res. 9: 19-25.

Scott, J.W., Hawley, S.A., Green, K.A., Anis, M., Stewart, G., Scullion, G.A., Norman, D.G., and Hardie, D.G. (2004). CBS domains form energy-sensing modules whose binding of adenosine ligands is disrupted by disease mutations. J Clin Invest. 113: 274-284.

Selhub, J. (1999). Homocysteine metabolism. Ann. Rev. Nutr. 19: 217-246.

Sen, S., Yu, J., Yamanishi, M., Schellhorn, D., Banerjee, R. (2005). Mapping Peptides Correlated with Transmission of Intrasteric Inhibition and Allosteric Activation in Human Cystathionine $\beta$-Synthase. Biochemistry. 44: 14210-14216.

Sen, S. and Banerjee R. (2007). A Pathogenic Linked Mutation in the Catalytic Core of Human Cystathionine $\beta$-Synthase Disrupts Allosteric Regulation and Allows Kinetic Characterization of a Full-Length Dimer. Biochemistry. 46: 4110-4116.

Shan, X., and Kruger, W.D. (1998). Correction of disease causing CBS mutations in yeast. Nat. Genet. 19: 91-93.

Shan, X., Dunbrack, R.L., Chritopher, S.A. and Kruger, W.D. (2001). Mutations in the regulatory domain of cystathionine $\beta$-synthase can functionally suppress patientderived mutations in cis. Hum. Mol. Genet. 6: 635-643.

Strambini, G. B., Cioni, P.,Cook, P. F. (1996). Tryptophan luminescence as a probe of enzyme conformation along the O-acetylserine sulfhydrylase reaction pathway, Biochemistry. 35: 8392-8400

Tai, C.H. and Cook, P.F. 2001. Pyridoxal 5'-phosphate-dependent $\alpha, \beta$-elimination reactions: mechanism of $O$-acetylserine sulfhydrylase. Acc. Chem. Res. 34: 49-59.

Taoka, S., Ohja, S., Shan, X., Kruger, W.D., and Banerjee, R. (1998). Evidence for hememediated redox regulation of human cystathionine $\beta$-synthase activity. $J$. Biol Chem. 273: 25179-25184 
Taoka, S., Widjaja, L. Banerjee, R. (1999). Characterization of the heme and pyridoxal phosphate cofactors of human cystathionine beta synthase reveals nonequivalent active sites. Biochemistry. 38: 13155-13161.

Taoka, S., Lepore, B.W., Kabil, O., Ojha, S., Ringe, D., Banerjee, R. (2002). Human Cystathionine $\beta$-Synthase Is a Heme Sensor Protein. Evidence That the Redox Sensor Is Heme and Not the Vicinal Cysteines in the CXXC Motif Seen in the Crystal Structure of the Truncated Enzyme. Biochemistry. 41:10454-10461

Toney, M.D. (2005). Reaction specificity in Pyridoxal phosphate enzymes. Archives of Biochemistry and Biophysics. 433. 279-287

Tseng, P.Y., Bennetts, B., Chen, T.Y. (2007). Cytoplasmic ATP inhibition of CLC-1 is enhanced by low pH. J. Gen. Physiol. 130: 217-221.

Vogel, H.J., and Bonner, D.M. (1956). Acetylornithinase of Escherichia coli: partial purification and some properties. J. Biol. Chem. 218: 97-106

Weiner, M.P., Costa, G.L., Schoettlin, W., Cline, J., Mathur, E., Bauer, J.C. (1994). Sitedirected mutagenesis of double-stranded DNA by the polymerase chain reaction. Gene 151: 119-23.

Wellhauser, L., Kuo, H.H., Stratford, F.L., Ramjeesingh, M., Huan, L.J., Luong, W., Li, C., Deber, C.M., Bear, C.E. (2006). Nucleotides bind to the C-terminus of ClC-5. Biochem. J. 398: 289-294 .

Woehl, E. U., Tai, C. H., Dunn, M. F., Cook, P. F. (1996). Formation of the alphaaminoacrylate immediate limits the overall reaction catalyzed by $\mathrm{O}$-acetylserine sulfhydrylase, Biochemistry. 35: 4776-4783.

Yang, F., Larry G. Moss, and George N. Phillips, Jr. (1996). The Molecular basis of green fluorescent protein. Nature Biotechnology. 14: 1246-1251

Yano, T., Kuramitsu, S., Tanase, S., Morino, Y., and Kagamiyama, H. (1992). Role of Asp222 in the catalytic mechanism of Escherichia coli aspartate aminotransferase: the amino acid residue which enhances the function of the enzyme-bound coenzyme pyridoxal 5'-phosphate. Biochem. 31: 5878-5887.

Yap, S. and Naughten, E. (1998). Homocystinuria due to cystathionine beta-synthase deficiency in Ireland: 25 years' experience of a newborn screened and treated population with reference to clinical outcome and biochemical control. $J$ Inherit Metab Dis. 21:738-47

Zhang, R., Evans, G., Rotella, F.J., Westbrook, E.M., Beno, D., Huberman, E., Joachimiak, A, and Collart, F.R. (1999). Characteristics and crystal structure of bacterial inosine-5-monophosphate dehydrogenase. Biochemistry 38: 4691-4700. 